\title{
Expressive Writing as a Therapeutic Process for Drug Dependent Women
}

Meshberg Cohen Sarah

Virginia Commonwealth University

Follow this and additional works at: https://scholarscompass.vcu.edu/etd

Part of the Psychology Commons

() The Author

\section{Downloaded from}

https://scholarscompass.vcu.edu/etd/2013

This Dissertation is brought to you for free and open access by the Graduate School at VCU Scholars Compass. It has been accepted for inclusion in Theses and Dissertations by an authorized administrator of VCU Scholars Compass. For more information, please contact libcompass@vcu.edu. 
(C) Sarah Meshberg-Cohen, 2009

All Rights Reserved 


\section{EXPRESSIVE WRITING AS A THERAPEUTIC PROCESS FOR DRUG DEPENDENT WOMEN}

A dissertation submitted in partial fulfillment of the requirements for the degree of Doctor of Philosophy at Virginia Commonwealth University.

by

Sarah Meshberg-Cohen

M.S., Virginia Commonwealth University, 2006

B.S., Skidmore College, 2002

Director: Dace S. Svikis, Ph.D.

Professor, Department of Psychology

Virginia Commonwealth University

Richmond, Virginia

May, 2010 


\section{Acknowledgements}

I wish to thank a number of people for helping me achieve the completion of this thesis. First, I would like to acknowledge and thank the National Institute on Drug Abuse (NIDA) and the VCU Institute for Women's Health (IWH) for the funding to carry out and complete this project. Second, I would like to thank my advisor and committee chair, Dr. Dace S. Svikis, for her advice, guidance, and mentoring throughout this project. More importantly, I would like to thank her for helping foster my excitement about research. I would also like to thank my committee members, Drs. Wendy Kliewer, James P. McCullough, Jr., Anika Alvanzo, and Diane Langhorst, for their suggestions and advice during the completion of this dissertation. More personally, I wish to thank my husband, Matt, for the unconditional love, support, and encouragement he has provided during my graduate school career so far. He has been extremely understanding, helpful, and patient, as I have focused on my professional goals.

This dissertation is dedicated to my husband Matt and my children Lucas and Tessa. You make every day wonderful, and I could not imagine my world without you. I love you. 
Table of Contents

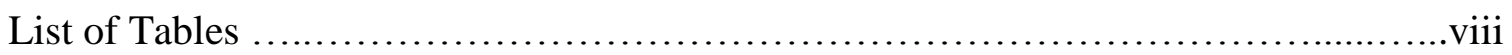

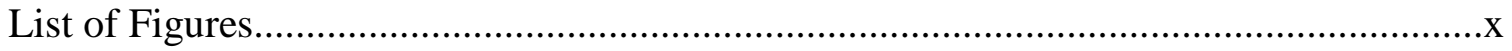

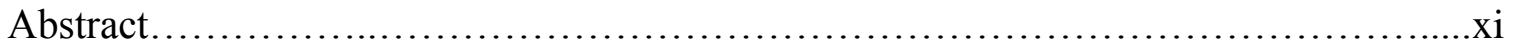

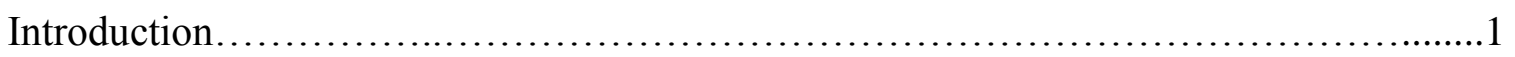

Review of the Literature.........................................................

Substance Use Disorders and Trauma ..........................................................

Substance Use Disorders ....................................................................

Substance Use Disorders and Women ............................................6

Trauma and Posttraumatic Stress Disorder (PTSD) ............................. 9

Substance Use Disorders and Trauma among Women..........................11

Childhood Trauma and Women with Substance Use Disorders...............12

Adult Trauma and Women with Substance Use Disorders .....................13

Domestic Violence and Women with Substance Use Disorders ...............17

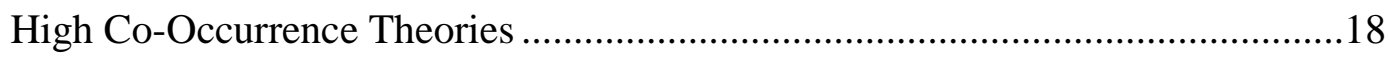

Self-Medication Theory ................................................................ 19

High-Risk and Susceptibility Theory.............................................21 
Third Factor Theory

Implications of Co-Occurring Trauma and SUDs ..........................................24

Trauma and Health among Women with SUDs ..................................24

Clinical Profiles among Women with SUDs and Trauma ......................25

Importance of Addressing Trauma in SUD Treatment..........................29

Assessing Trauma in SUD Treatment...............................................33

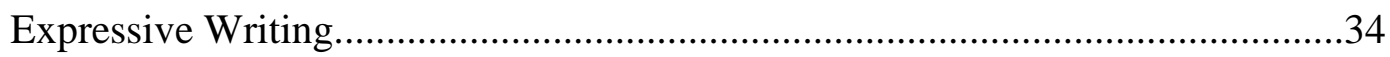

Expressive Writing and Disclosure .................................................34

Expressive Writing with Different Populations .....................................37

Mechanisms through which Expressive Writing Works ........................40

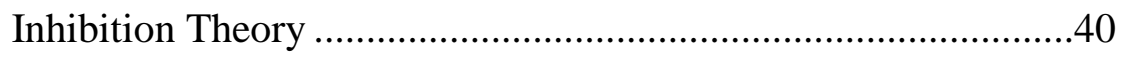

Cognitive Processing Theory ..............................................41

Self-Regulation Theory ...................................................42

Statement of the Problem and Hypotheses ............................................................ 44

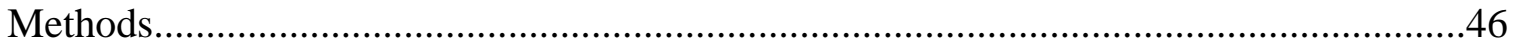

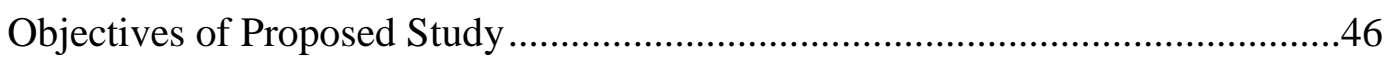

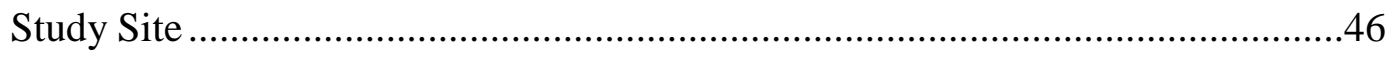

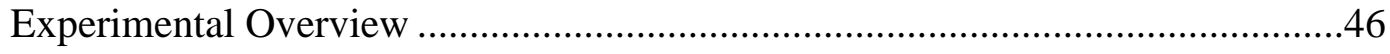


Participants.

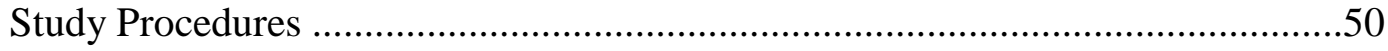

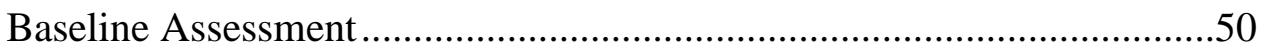

Expressive Writing Intervention ..............................................................53

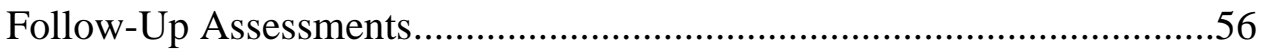

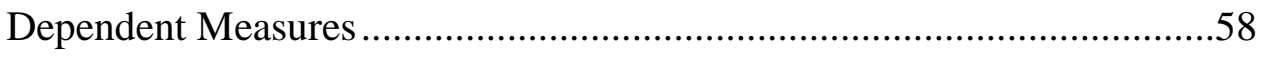

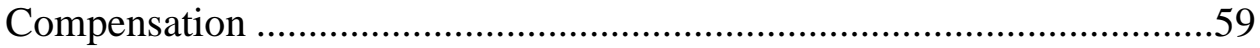

Critical Methodological Issues ................................................................59

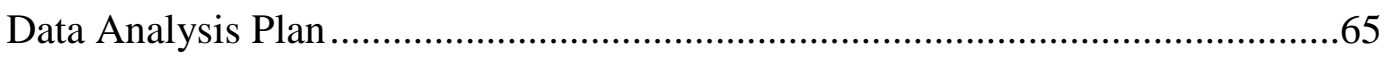

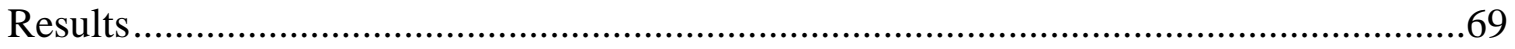

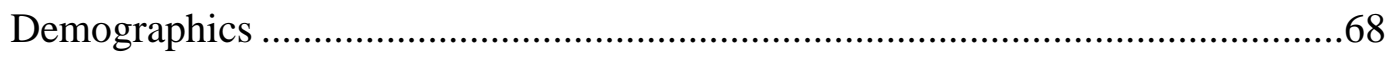

Data Analysis ............................................................................................

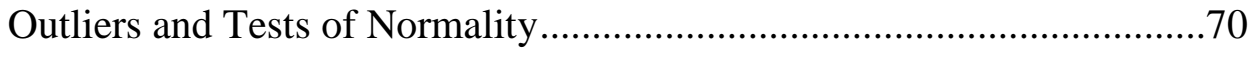

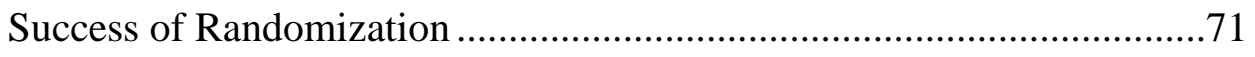

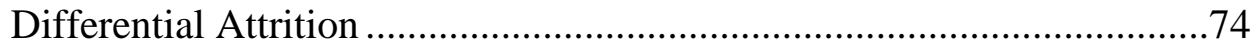

Writing Session Attrition ......................................................

Two Week Follow-Up Attrition.................................................75

One Month Follow-Up Attrition...............................................77 
Descriptive Analyses

Trauma and PTSD among Residential SUD Women ................................77

Various indices of Distress among Residential SUD Women....................80

Levels of Depression and Common Physical Symptoms among

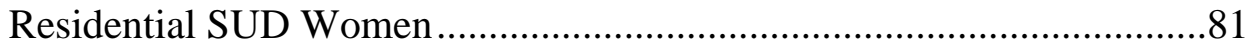

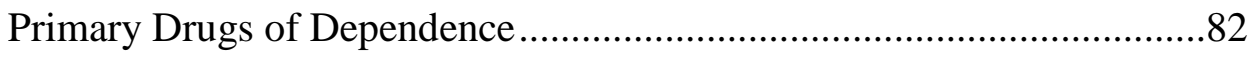

Brief Substance Craving Scale.................................................................83

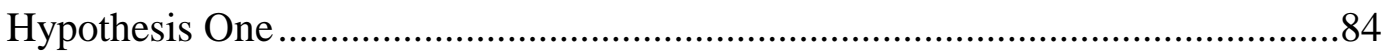

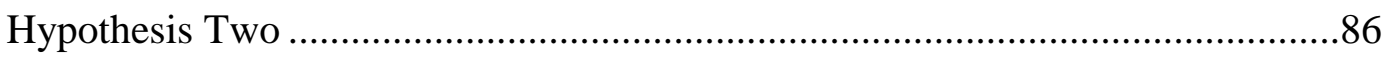

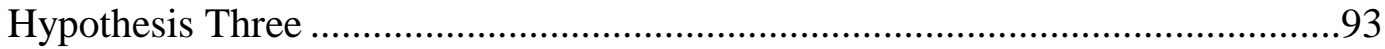

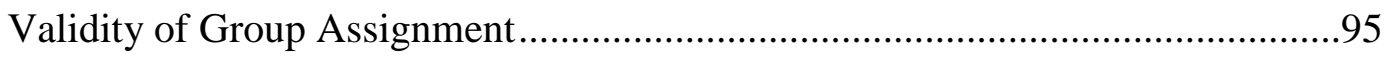

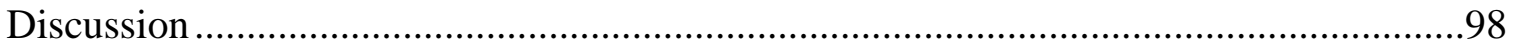

Epidemiology of Trauma and PTSD in Women with SUDs .................................98

Clinical Profiles and Treatment Response in SUD Women with and without

PTSD

Trauma and Health among Women with SUDs .....................................101

Clinical Profiles among Women with SUDs and Trauma .......................102

Depression among Women with SUDs and Trauma ................................103 
Drug Craving and Drugs of Dependence among Women with SUDs and

Trauma 105

Shorter Term and Longer Term Effects of Expressive Writing in SUD Women 107

Trauma Symptom Severity 107

Depression.

Physical Health Problems

Anxiety Index from Brief Symptom Inventory 115

Somatic Index from Brief Symptom Inventory 116

Remaining BSI Indices 116

Expressive Writing at One Month Follow-Up in a Residential SUD Treatment

Program

Effects of Expressive Writing on Immediate and Longer-Term Levels of

Distress.

Expressive Writing with Drug Dependent Women

Study Implications, Future Directions, and Applications 122

Study Limitations

References 130

Appendices. 145

Vita. .156 vii 


\section{List of Tables}

Table

1. Participant Characteristics: Expressive Writing $(n=82)$ and Control Writing $(n=67)$

2. Trauma and PTSD: Expressive Writing $(n=82)$ and Control Writing $(n=67) \ldots .78$

3. Different Types of Trauma Events: Expressive Writing $(n=82)$ and Control Writing $(n=67)$

4. Brief Symptoms Inventory: Expressive Writing $(n=82)$ and Control Writing $(n=$ 67)

5. Center for Epidemiological Studies - Depression Scale (CES-D) and Pennebaker Inventory of Limbic Languidness

6. DSM-IV Substance Dependence Diagnosis (current): Expressive Writing $(n=82)$ and Control Writing $(n=67)$

7. Brief Substance Craving Scale: Expressive Writing $(n=82)$ and Control Writing $(n=67)$.

8. Brief Symptom Inventory: PTSD Diagnosis $(n=81)$ and No PTSD Diagnosis $(n$ $=68)$.

9. Effects of Expressive Writing versus Control Writing on Short-term and Longerterm Follow-Up

10. Effects of Expressive Writing versus Control Writing on BSI Index Scores: Shortterm and Longer-term Follow-Up...

11. Negative Affect: Pre-Post Writing and Follow-up .....

12. Essay Evaluation Measure scores (average item answer) for Expressive Writing ( $n$ $=77)$ and Control Writing $(n=64)$

13. Follow-Up Questionnaire at One Month Follow-Up: Expressive Writing $(n=70)$ and Control Writing $(n=50)$ 
14. Follow-Up Questionnaire: 10 randomly selected Quotes from Each Writing

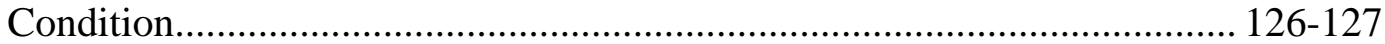




\section{List of Figures}

Figure Page

1. Flow Chart of Participants Through Study .......................................71 


\title{
Abstract \\ EXPRESSIVE WRITING AS A THERAPEUTIC PROCESS FOR DRUG DEPENDENT WOMEN
}

\author{
by Sarah Meshberg-Cohen, M.S.
}

A dissertation submitted in partial fulfillment of the requirements for the degree of Doctor of Philosophy at Virginia Commonwealth University.

Virginia Commonwealth University, 2009

Major Director: Dace S. Svikis, Ph.D., Professor, Department of Psychology

Women with Substance Use Disorders (SUD) have high rates of trauma and PTSD, which is linked to greater physical and mental health problems and poorer SUD treatment outcomes. While research affirms trauma should be addressed during SUD treatment, the majority of addiction programs do not offer such services. One promising intervention is Pennebaker's expressive writing paradigm, which includes disclosure of traumatic/stressful experiences through 20-minute writing sessions over 3-5 consecutive days. While expressive writing has been linked to improvements in mental and physical health, the intervention has not been studied in persons with SUDs. The present study was a randomized clinical trial comparing expressive writing to control (neutral topic) writing. Conducted in a residential SUD program for women, the study: 1) compared psychological and physical health profiles in SUD women with and without co-morbid PTSD and/or trauma; 2) compared 2-week and 1-month outcomes for the experimental and control groups; and 3) examined immediate and more distal levels of psychological distress following expressive writing. Study participants were $N=149$ women 
randomized to either the expressive writing or control writing condition. All women completed 20-minute writing sessions daily for 4 consecutive days. As predicted, SUD women reported high rates of trauma and PTSD, and those with such co-morbidities had more severe psychological and physical health problems. At 2-week follow-up, expressive writing participants showed greater reductions in post-traumatic symptom severity and anxiety scores than control writing participants. While no group differences were found at 1-month follow-up, this was due largely to significant improvements for both groups over the course of residential treatment. Finally, expressive writing participants showed increased negative affect immediately after each writing session, but there were no differences in pre-writing negative affect scores between groups the following day. By the final writing session, participants were able to write about traumatic/stressful events without having a spike in negative affect. Study results suggest expressive writing may be a brief, cost effective, adjunct to SUD treatment. Furthermore, expressive writing, when implemented in a residential SUD setting, appears safe, and warrants further study as a strategy for addressing trauma and PTSD in tandem with SUD treatment. 


\section{Introduction}

Epidemiological surveys and clinical studies point to overwhelming rates of interpersonal trauma in the lives of women with Substance Use Disorders (SUDs). As many as $80 \%$ of women needing treatment for drug problems report lifetime histories of physical and/or sexual assault (Hien, Cohen, \& Campbell, 2005), and many display Posttraumatic Stress Disorder (PTSD) symptomatology (Gilbert, El-Bassel, Schilling, \& Friedman, 1997; Brown, Recupero, Stout, 1995; Brown, Stout, \& Mueller, 1999). In these settings, $55 \%$ to $99 \%$ of women report at least one lifetime trauma (Najavits, Weiss, \& Shaw, 1997). Trauma and addiction comorbidity studies consistently reveal these women are likely to have poorer health and increased disability, more severe clinical profiles, and worse treatment adherence, than those without traumatic experiences or PTSD (Najavits, 2006). Greater rates of revictimization are found among females with SUDs compared to those without SUDs. While trauma has traditionally not been incorporated into SUD treatment programs, studies indicate that addressing trauma during SUD treatment may improve treatment outcomes (Hien, Cohen, Miele, Litt, Capstick, 2004; Batten \& Hayes, 2005; Brady, Dansky, Back, Foa, Carroll, 2001; Najavits, 2006).

Accumulating evidence over the past two decades indicates that disclosure of traumatic or stressful experiences through expressive writing has widespread mental and physical health benefits. Past studies have demonstrated the efficacy of expressive writing as a brief therapeutic intervention, with significant reductions in distress (Smyth, 1998), and improved physical (Pennebaker, Mayne, \& Francis, 1997) and psychological 
health (Lepore \& Smyth, 2002). Beneficial effects related to writing about emotional experiences have been shown in areas as varied as health, achievement, and general wellbeing (Slatcher \& Pennebaker, 2006).

Although much attention has focused on the benefits of Pennebaker's expressive writing paradigm, to date, expressive writing has not been examined in populations affected by SUDs. Expressive writings' efficacy, efficiency (15-20 minutes per session over three to five consecutive days), and cost-effectiveness (the cost of a notebook), suggest that writing as a means for disclosing traumatic experiences may be a powerful adjunct to traditional alcohol and drug treatment, particularly in SUD treatment settings that do not routinely screen for trauma and PTSD and therefore, do not specifically focus on such issues during treatment. To date, however, its usefulness as an intervention for SUD populations is unknown.

The purpose of the present study is to examine whether Pennebaker's expressive writing paradigm is beneficial for women currently undergoing SUD treatment. The expressive writing paradigm includes writing about an assigned topic daily for 20 minute sessions over four consecutive days. If expressive writing improves outcomes for women with SUDs, these findings would support its use as a brief, cost effective, adjunct to current SUD treatment.

The present study begins with an extensive review of the literature on the cooccurrence between trauma and substance abuse/dependence in women. Next, theories behind the co-occurrence of trauma and SUDs, as well as the physical and psychological health implications are reviewed, with a discussion about the importance of addressing 
trauma in conjunction with substance abuse treatment services. Finally, expressive writing and disclosure as a therapeutic intervention is discussed, with a review of the various populations that expressive writing has been used with, and the possible mechanisms through which expressive writing may yield positive outcome results.

Specific Aim 1: Investigate physical and mental health problems, as well as the relationships between PTSD and/or trauma and physical and mental health problems.

Hypothesis 1: Trauma symptom severity and/or PTSD will be associated with more significant physical health and psychological problems upon admittance to residential SUD treatment.

Specific Aim 2: In a randomized clinical trial, the present study will compare outcomes for a treatment-based sample of substance abuse/dependent women assigned to either a written disclosure condition (emotional topic) or a control writing condition (neutral topic).

Hypothesis 2: At two week and one month follow-ups, participants randomized to the expressive writing condition will show greater reductions in post-traumatic symptom severity, health symptoms, psychological distress, depressive symptoms, and drug cravings than participants randomized to the control writing condition.

Specific Aim 3: Examine the influence of the expressive writing task on both immediate and longer-term levels of psychological distress.

Hypothesis 3: Women randomized to the expressive writing group will show higher levels of immediate distress (measured immediately after each writing session) 
and lower levels of longer-term distress (measured at two and one month post-baseline assessment) relative to those in the control group. 


\section{Review of the Literature}

\section{Substance Use Disorders and Trauma}

\section{Substance Use Disorders}

A Substance Use Disorder (SUD) is a subcategory of substance-related disorders that includes both Substance Dependence and Substance Abuse (DSM-IV-TR; American Psychiatric Association, 2000). It includes disorders related to drugs of abuse ranging from alcohol to heroin.

According to the Diagnostic and Statistical Manual, Fourth Edition, Text Revision (DSM-IV-TR; American Psychiatric Association, 2000), Substance Dependence involves a cluster of cognitive, behavioral, and physiological symptoms indicating that the individual continues to use the substance despite considerable substance-related problems. Substance Dependence involves a maladaptive pattern of substance use causing clinically significant impairment or distress, as revealed by three (or more) of the following within a 12-month period: 1) tolerance; 2) withdrawal; 3) taking larger amounts of the substance over a longer period of time than intended; 4) persistent desire or unsuccessful efforts to cut down or control use; 5) great deal of time spent in activities necessary to obtain, use, or recover from the effects of the substance; 6) important occupational, recreational, or social activities given up because of substance use; 7) recurrent use despite knowledge of having physical or psychological problems likely caused or exacerbated by the substance.

Unlike the criteria for Substance Dependence, Substance Abuse does not include tolerance, withdrawal or a pattern of compulsive use, but rather it includes only the 
harmful consequences of repeated use. According to the DSM-IV-TR (American Psychological Association, 2000), the main characteristic of Substance Abuse is a maladaptive pattern of substance use exhibited by recurring and significant adverse consequences related to the continual use of substances. It involves a maladaptive pattern of substance use causing clinically significant impairment or distress, as exhibited by at least one of the following within a 12-month period: 1) failure to fulfill major role obligations at school, work, or home due to recurring substance use; 2) repeated substance use in situations that are physically hazardous (e.g. driving, operating machinery); 3) repeated substance-related legal problems; 4) recurring substance use despite having continual or repeated interpersonal or social problems caused or exacerbated by the effects of the substance (American Psychological Association, 2000). In order to meet criteria for Substance Abuse, criteria for Substance Dependence must never have been met.

Throughout this proposal, we refer to SUDs, which include either Substance Dependence or Substance Abuse of alcohol and other drugs.

\section{Substance Use Disorders and Women}

Historically, SUDs were thought to primarily affect men, and the majority of SUD studies were conducted predominantly with male subjects. However, the gap between the proportion of male and females with SUDs has been narrowing, and recent studies show an increase in numbers of women with drug abuse problems (Gutierres \& Van Puymbroeck, 2006). Even though women are less likely than men to have opportunities to use drugs, when given the opportunity to use drugs for the first time, women and men 
are equally likely to do so and to progress from initial use to addiction (Van Etten \& Anthony, 2001). While rates of SUDs remain higher in men versus women, about 7 million American women between 15 and 54 years of age have a lifetime SUD, with over 4.4 million American women considered to be currently drug dependent. Of these 4.4 million American women, 2.5 million meet criteria for current drug abuse, representing over 30 percent of the illicit-drug-using population (Turner \& Wallace, 2003; United Nations Office on Drugs and Crime; UNODC, 2004).

Although SUDs are a rising health concern among females, women are underrepresented in most SUD treatment programs (Greenfield, et al., 2007). Because most drug treatment programs were originally created to address issues common to males, the treatment and research communities have recently recognized the need to identify and better understand gender differences in the etiology of substance use and substance use treatment needs and treatment utilization, with particular attention to the effectiveness of various SUD treatment services for women (Pelissier \& Jones, 2005).

Recent research focused on women with SUDs has revealed numerous sex differences (Lynch, Roth, \& Carroll, 2002). Females frequently encounter a greater number of barriers to treatment-seeking than their male counterparts. Main barriers to treatment among women includes concerns about childcare, transportation, social stigma and labeling, a lack of awareness of the variety of treatment choices, problems with the confrontational models used by some SUD programs, time and economic costs of treatment, and stereotypical ideas of clients of treatment services (Copeland, 1997). Such obstacles may contribute to the fact that by the time they present for treatment, women 
generally present with higher levels of addiction severity and more severe co-morbidities than men (Copeland, 1997). Women are also more likely than men to seek treatment for drug abuse problems in a mental health facility than in a SUD treatment program (Lynch, Roth, \& Carroll, 2002). In addition, studies suggest that women generally show greater psychological distress, greater addiction severity, more medical problems, and fewer occupation marketable skills than men at the time of treatment entry (Arfken, Klein, di Menza, \& Schuster, 2001).

Studies addressing gender differences in post-treatment outcomes have revealed mixed results, with some studies showing women and men with comparable outcomes (Alterman, Randall, McClellen, 2000; Gerstein and Johnson, 2000), while other studies support one gender over the other (Arfken, et al., 2001; Grella, Scott, Foss, Joshi, Hser, 2003; Gutierres \& Van Puymbroeck, 2006; Niv \& Hser, 2007). For instance, Alterman and colleagues (2000) compared 7-month post-treatment entry of nine community programs $(N=294 ; n=145$ female $)$ and found few gender difference outcomes.

On the other hand, some studies find that, compared to men, women fare worse in drug SUD treatment programs, and have significantly lower rates of treatment entry, treatment retention, and successful treatment completion (Arfken, et al., 2001; Grella, et al., 2003; Gutierres \& Van Puymbroeck, 2006). For instance, in addition to finding more severe problems among women than men at assessment, Arfken and colleagues (2001) examined a publicly funded SUD treatment system $(N=2,741)$, and found that women had significantly lower treatment completion rates and 30-day retention in treatment than men. 
Given the recognized obstacles and problems that women have in substance use treatment programs, it is important to better understand the issues that may account for these difficulties (Gutierres \& Van Puymbroeck, 2006). Types of issues to be addressed during treatment with females with SUDs should acknowledge the comprehensive range of women's problems (Pelissier \& Jones, 2005). One of the most commonly documented factors by researchers is the link between trauma and drug addiction in the lives of women (Gutierres \& Van Puymbroeck, 2006).

\section{Trauma and Posttraumatic Stress Disorder (PTSD)}

A traumatic event is defined as an emotionally disturbing and distressing event beyond the range of normal human experience, which can be experienced first-hand or witnessed, and can include child abuse, sexual assault, domestic violence, military combat, and a host of other violent incidents (MIMH fact sheet, 2004). While men are generally more likely to be exposed to traumatic events during their lifetime, women are more likely to endure chronic high impact trauma such as physical abuse, childhood sexual violence, and neglect (Stewart, Ouimette, \& Brown, 2002). Not all people who experience traumatic events go on to develop PTSD; however, once exposed to trauma, women are about twice as likely to develop PTSD compared to men, and the traumatic events experienced by women are also qualitatively different from those experienced by men (Stewart, et al., 2006). For instance, data from the National Comorbidity Survey (NCS), a nationally representative U.S. epidemiological study of people ages 15 to 54 years old $(N=5388)$, revealed that approximately $61 \%$ of men were exposed to trauma 
compared to $51 \%$ of women, however, only $5 \%$ of men compared to $10 \%$ of women met DSM-IV diagnostic criteria for PTSD (Kessler, et al., 1995).

According to the DSM-IV, PTSD is a psychiatric disorder, which a person may develop after experiencing or witnessing a severe or life-threatening event (American Psychiatric Association, 2000). The essential characteristics of PTSD involve the development of symptoms following the experience of an acute stressor involving direct personal experience of an event that includes actual or threatened death or serious injury, or other threat to one's physical integrity; or observing an incident that involves injury, death, or a threat to the physical integrity of another person; or learning about serious harm, a sudden or violent death, or threat of injury or death experienced by a close associate or family member (Criterion A1). The individual's reaction to the incident must include helplessness, extreme fear, or horror (Criterion A2) (American Psychiatric Association, 2000). The characteristic symptoms resulting from the exposure to the extreme trauma include persistent re-experiencing (e.g., flashbacks, hallucinations, illusions, dreams, etc.) (Criterion B), avoidance/numbing (e.g., avoiding thoughts, feelings, activities, places, or people or conversations associated with the trauma, etc.) (Criterion C), and hyperarousal (e.g., irritability/outbursts of anger, hypervigilance, exaggerated startle response, etc.) (Criterion D) relating to the original trauma, which must be of at least one month duration (Criterion E) and associated with impaired functioning (Criterion F) (Reynolds, et al., 2005; American Psychiatric Association, 2000). 
There is currently a lot of heterogeneity in the literature looking at trauma versus PTSD. For the purposes of this proposal, the review of the literature will specify whether the particular study identifies PTSD or trauma.

\section{Substance Use Disorders and Trauma among Women}

Epidemiological surveys and clinical studies point to overwhelming rates of interpersonal trauma in the lives of women with SUDs. The high co-occurrence of traumatic stress exposure and SUDs is increasingly evident. As many as $80 \%$ of women needing treatment for drug problems report lifetime histories of physical and/or sexual assault (Dansky, Saladin, Brady, Kilpatrick, \& Resnick, 1995; Hien, Cohen, \& Campbell, 2005), and many display PTSD symptomatology (Brown, Recupero, Stout, 1995; Brown, Stout, \& Mueller, 1999; Gilbert, El-Bassel, Schilling, \& Friedman, 1997).

Epidemiological studies reveal that SUDs are 3.1 to 4.5 times more likely among women with PTSD compared to those without PTSD (Kessler, Sonnega, Bromet, Hughes, \& Nelson, 1995). Rates of current PTSD among women in substance treatment settings range from $30 \%$ to $59 \%$ (Najavits, et al., 1997), compared to PTSD rates in the general population ranging from $1 \%$ to $9 \%$ (Kessler, et al., 1995). In addiction treatment settings, $55 \%$ to $99 \%$ of women report at least one lifetime trauma (Najavits, et al., 1997). Greater rates of revictimization are also found among females with SUDs compared to those without SUDs (Dansky, Brady, \& Saladin, 1998). The proposed study will determine rates of trauma and PTSD-related symptomatology in a residential treatment sample of SUD women. 


\section{Childhood Trauma and Women with Substance Use Disorders}

Research suggests that the majority of women with comorbid SUD and PTSD are victims of childhood trauma (Brown \& Wolfe, 1994), and are likely to experience repeated traumatic events in adulthood (Cohen \& Hien, 2006). Female drug users, more often than males, are found to have high rates of violent victimization as children and as adults (Guiterres \& Van Puymbroeck, 2006). These trends are seen across various drug using populations. For instance, one study examining residential male and female drug abuse patients $(N=146 ; n=72$ women $)$ found that $79 \%$ of women versus $41 \%$ of men reported childhood emotional abuse, $51 \%$ of women versus $23 \%$ of men reported childhood physical abuse, and $52 \%$ of women versus $7 \%$ of men reported childhood sexual abuse (Gutierres \& Todd, 1997). In a residential drug treatment facility $(N=178)$, $84 \%$ of female and male patients reported a history of childhood sexual or physical assault (Cohen \& Densen-Gerber, 1982). In a prison population, childhood abuse (sexual abuse and/or physical abuse and/or neglect) was found to be a significant predictor of adult arrests for drug and alcohol related offenses for female prisoners, but not for male prisoners (Ireland \& Widom, 1994).

Women are also more likely than men to report that they started using drugs in order to mitigate trauma-related symptoms connected with abuse (Sheridan, 1996; Gutierres \& Todd, 1997; Woodhouse, 1992), and are more likely to experience repeated trauma in later life (Dansky, Brady, \& Saladin, 1998). Studies consistently find that women who have experienced childhood sexual abuse are more vulnerable than nonvictims to be sexually abused as adults, and are more likely to endure physical abuse later 
in life by their husbands and partners (Desai, Arias, Thompson, \& Basile, 2002; Guterres \& Van Puymbroeck, 2006). Even after controlling for family background variables (e.g., parental alcohol problems) and treatment condition, rates of childhood victimization are shown to be higher for women in alcohol-related treatment (e.g., outpatient alcoholism clinics) compared to women in treatment for non-alcohol related problems (e.g., outpatient mental health clinics) (Miller, Downs, \& Testa, 1993). Of note, while rates of reported childhood trauma among women in SUD treatment programs is high, there is reason to believe that the real incidence is even higher, since studies that use multiple assessments show that some women who do not identify the abuse at intake, admit experiencing abuse later in treatment (Briere \& Conte, 1993; Gutierres \& Van Puymbroeck 2006).

\section{Adult Trauma and Women with Substance Use Disorders}

In addition to the high rates of child abuse reported among women seeking SUD treatment, it is also clear that these women experience a significant amount of trauma during adulthood. For instance, Gilbert and colleagues (1997) examined adult victimization among women in methadone treatment $(N=151)$ and found that $88.7 \%$ had experienced physical abuse, $71.1 \%$ experienced having their life threatened, 25.3\% experienced sexual abuse, and $42.9 \%$ experienced both physical abuse and having their life threatened. While not all individuals with trauma go on to have PTSD, the majority of individuals in SUD treatment have significant trauma histories.

In a study comparing SUD patients with and without comorbid PTSD, Brown and colleagues (1999) found that $95 \%$ of participants $(N=90)$, regardless of PTSD diagnosis, 
had been exposed to significant trauma during their lifetime. Reported traumas included catastrophic death of a loved one (e.g., homicide or suicide; 65\%), childhood physical abuse (40\%), childhood forced sexual touching (40\%), and adulthood physical abuse (38\%). Women reported significantly more types of traumas than men $(M=4.0$ vs $M=$ 2.8), and were significantly more likely than men to report physical abuse during adulthood (59\% vs 14\%) and forced sex during adulthood (31\% vs 5\%; Brown, Stout, \& Mueller, 1999).

Oftentimes, the traumatic events experienced by women are qualitatively different from those experienced by men, and women may be affected differently than men by similar events (Stewart, Ouimette, \& Brown, 2002). Najavits and colleagues (1998) examined rates of lifetime trauma and current PTSD symptomatology among treatmentseeking cocaine dependent outpatients $(N=122 ; n=43$ females) from the National Institute on Drug Abuse Collaborative Cocaine Treatment Study, and found a large number of traumatic events $(M=5.7)$. Women were significantly more likely than men to meet criteria for past month (current) PTSD (30\% versus 15\%, respectively). Results also indicated gender differences in the types of trauma leading to PTSD, where women were more likely than men to experience physical and sexual abuse $(M=1.4$ versus $M=$ 0.9 , respectively), while men were more likely to report general disasters and crimerelated traumas than women ( $M=3.9$ versus $M=2.0$, respectively).

Similarly, Coffey and colleagues (1998) examined lifetime exposure to trauma among SUD patients $(N=118 ; n=67$ women $)$ and found that women were more likely than men to report rape (37\% of women versus $6 \%$ of men) and other sexual assault ( $25 \%$ 
of women versus $11 \%$ of men), while men were significantly more likely than women to be exposed to combat trauma ( $10 \%$ of men versus $0 \%$ of women). Another study examining residential drug dependent patients ( $N=100 ; n=50$ females), found that over half $(66 \%)$ of the sample had a sexual or physical assault history, with women being significantly more likely than men to have experienced sexual assault, serial assault, and familial assault (Grice, Brady, Dustan, Malcolm, \& Kilpatrick, 1995). Half of the assault victims, but none of those who did not experience assault, met criteria for PTSD.

Findings also revealed that patients with a childhood abuse history started abusing drugs at an earlier age compared to those without a childhood abuse history (Grice, et al., 1995).

In a study focusing cocaine dependent patients ( $N=91 ; n=33$ females), Dansky and colleagues (1999) found that $86 \%$ of patients reported a history of physical assault. There were no significant differences between men and women in terms of their likelihood of being physically assaulted; however, women were more likely than men to have been assaulted by a partner ( $76 \%$ versus $29 \%$, respectively). Women were also more likely than men to fear death or serious harm during aggravated assault without a weapon (94\% versus 56\%, respectively) and during simple assault (82\% versus $18 \%$, respectively) (Dansky, Bryne, \& Brady, 1999). In another study examining residential SUD patients ( $N=84 ; n=36$ females), Brown and colleagues (1995) found that women were more likely than men to have experienced physical assault/abuse (31\% versus $6 \%$ ), sexual assault/abuse ( $25 \%$ versus $4 \%$ ), and rape (25\% versus $2 \%)$; women were also significantly more likely than men to meet criteria for lifetime PTSD (43\% versus $12 \%$ ). 
While high rates of physical assault and PTSD are found among men and women with SUDs, women may be affected differently than men. For instance, in an evaluation of gender differences in criminal victimization and crime-related PTSD rates among inpatient SUD adults ( $N=95 ; n=61$ females $)$, approximately $90 \%$ reported a history of physical and/or sexual assault (Dansky, Brady, Saladin, Killeen, et al., 1996). Structured interviews were used to evaluate a lifetime history of crime-related victimization, which included sexual molestation, attempted sexual assault, completed rape, aggravated assault, and other physical assaults (e.g., serious physical attacks experienced prior to age 18 that were perpetrated by a guardian) and PTSD. While non-crime related events included serious accidents, natural disasters, homicide of a loved one, exposure to dead bodies, and witnessing the violent death of a person, in order to meet criteria for a crimerelated PTSD patients needed to have experienced at least one crime-related traumatic event and meet PTSD criteria. Results revealed that women were more likely than men to have experienced rape (59\% versus $21 \%$, respectively), and were also more likely than men to have felt that their life was in jeopardy during the rape (89\% versus $57 \%$, respectively). Overall, $53 \%$ of the women and men met diagnostic criteria for lifetime crime-related PTSD, with over $40 \%$ meeting criteria within the past 6 months (Dansky, et al., 1996).

Other studies have found that having experienced more than one significant lifetime trauma is associated with an increased rate of PTSD. For instance, Fullilove and colleagues (1993) looked at female drug users $(N=105)$ in treatment for SUDs, and found that almost all ( $n=104$ out of $N=105)$ of the women had experienced at least one 
significant trauma, with 59\% of these women meeting criteria for PTSD. This study also found that $97 \%$ of women with PTSD reported one or more violent trauma compared with 73\% of those without PTSD (Fullilove, et al., 1993).

\section{Domestic Violence and Women with Substance Use Disorders}

Women with childhood abuse histories are more likely than those without such histories to end up in violent relationships when they get older. Partner violence is a prevalent issue among women with SUDs, and up to $80 \%$ of women attending SUD treatment programs report a history of domestic violence (Bennett \& Lawson, 1994; North, Thompson, Smith, \& Kyburz, 1996). For instance, one study examining violence in the lives of homeless mothers in SUD treatment found that $84 \%$ reported childhood or adult victimization (North, et al., 1996). This study also revealed that while $60 \%$ of the women reported childhood physical or sexual abuse, $71 \%$ indicated adult victimization in the form of domestic violence or sexual abuse.

A recent longitudinal study by Salomon and colleagues (2002) showed that women who experienced adult partner violence were nearly three times more likely to report illegal drug use at follow up than those not exposed to partner violence. There was also a significant relationship between childhood abuse and domestic violence in later life, and the combination of childhood sexual abuse and resultant PTSD was a better predictor of substance abuse than either risk factor alone (Salomon, Bassuk, \& Hintington, 2002). While some drug abusing women who are in violent relationships end up in SUD treatment programs, others may seek help from domestic violence programs. A randomly selected sample of 74 programs for SUDs or domestic violence revealed that 
$60 \%$ of female drug abusers were victims of domestic violence, and $42 \%$ of women in domestic violence programs were abusing drugs (Bennett \& Lawson, 1994).

\section{High Co-Occurrence Theories}

The presence of either problem (SUD or PTSD) can increase the risk of developing the other disorder, and these findings are consistent across various kinds of traumas and substances of abuse (Najavits, Weiss, \& Shaw, 1997). While high comorbidity rates indicate that PTSD and SUDs are functionally related, the mechanisms underlying the development of this relationship is not clear (Back, Brady, Sonne, Verduin, Back, et al., 2006).

Three main pathways have been used to account for these high rates of comorbidity. While alcohol and drugs may be used to self medicate to avoid trauma reminders and related distress, the lifestyle of someone with drug use problems may predispose these individuals to endure traumatic events (Johnson, Striley, \& Cottler, 2006; Reynolds, et al., 2005). Another theory suggests that an independent third factor, such as genetic and/or environmental influences, may contribute to the high cooccurrence of both problems (Brown \& Wolfe, 1994; Xian, et al., 2000). The proposed study will examine whether trauma is related to drug craving among women in a residential SUD treatment facility.

\section{Self-Medication Theory}

The self-medication hypothesis suggests that people with trauma histories and related PTSD symptomatology use drugs in an effort to help them avoid or cope with upsetting symptoms and to reduce painful feelings or physiological sensations (Hien, 
Cohen, \& Campbell, 2005). Khantzian's (1985) proposed self-medication theory of addictive disorders theorizes that drugs are chosen for use according to their particular psychotropic effects (e.g., cocaine may alleviate depressive symptoms, while heroin may alleviate anxiety and aggressive feelings). Exposure to extreme trauma, particularly during childhood, may disrupt self-regulatory processes and cognitive systems, and such deficits have been associated with initiation and continuation of SUDs (Hien, Cohen, \& Campbell, 2005). Thus, self-medicating with drugs and alcohol can decrease the hyperarousal and numbing effects by masking symptoms of PTSD and providing temporary relief from dysregulated feelings (e.g., Khantzian \& Schneider, 1986).

In support of the self-medication hypothesis, a longitudinal study of adults aged 21 to $30(N=1007)$, who were initially assessed in 1989 and followed up 3 and 5 years later, revealed that preexisting PTSD signaled an increased risk of SUDs (hazards ratio $=$ 4.5; Chilcoat \& Breslau, 1998). Chilcoat and Breslau (1998) used a prospective design and analytic strategies for testing causal inference, and found that SUDs in individuals with PTSD might be the unplanned result of efforts to medicate symptoms. Similarly, an epidemiologic study, using a co-twin design, found that regardless of being raised in the same family upbringing, a twin who was exposed to childhood sexual abuse had a significantly elevated risk for SUDs when compared to her unexposed twin, supporting a causal interpretation of the association between childhood sexual abuse and SUDs (Kendler, et al. 2000). A longitudinal study by Kilpatrick and colleagues (1997) also found that sexual and physical abuse led to drug misuse, and that active drug use was associated with an increased risk of victimization. 
Some studies have found a direct causal pathway between childhood abuse and drug abuse. Schuck and Widom (2001), for example, used structural equation modeling in a sample of women $(N=582)$ who had endured childhood victimization, and found that childhood abuse played a causal role in alcohol symptoms among women, which was mediated by depression as well as the use of drugs or alcohol to cope. In another study, Brady and colleagues (1993) found that trauma and PTSD appeared prior to the onset of SUDs for the majority of women (78\%) and men (73\%), which revealed temporality data that are compatible with the self-medication theory for both genders. Finally, in a comprehensive review by Stewart (1996), results showed that individuals exposed to trauma, specifically those suffering from PTSD, were at a higher risk for developing alcohol problems than those not exposed, and that the most common explanation suggested to account for the comorbidity is that alcohol was being used to self-medicate symptoms of PTSD. While there is empirical support for the self-medication model (Hien, Cohen, \& Campbell, 2005), another pathway, such as the high-risk and susceptibility theory, has also been suggested.

\section{High-Risk and Susceptibility Theory}

The high-risk and susceptibility theory suggests that drug use and related highrisk behaviors increase the risk to traumatic encounters, thus circuitously enhancing the probability of trauma and PTSD-related symptomatology (Hien, Cohen, \& Campbell, 2005). This pathway suggests that drug abuse may precede PTSD. As women engage in the drug market, their dependence on drugs escalates and their lives generally become characterized by greater social chaos, where violence, sexual degradation, and fatality are 
elements of the drug life. Ongoing drug and crime interactions put women at high risk for assault, rape and death when compared to women who do not use drugs (Boyd, Hill, Holmes, \& Purnell, 1998).

Chronic drug use can also produce elevated levels of anxiety and arousal as well as sensitization of neurobiological stress systems, and may cause a higher level of susceptibility to the progression of PTSD after trauma exposure (Jacobsen, Southwick, \& Kosten, 2001). Thus, drug abusers may be more vulnerable to PTSD after a traumatic event due to impaired neurochemical or psychological systems stemming from persistent drug use (Hien, Cohen, \& Campbell, 2005). These associations between drug use and trauma do not necessarily suggest that SUDs always happen prior to exposure to traumatic events and PTSD, but rather, that there is a vicious cycle of events, which in many cases, can start with drug use (Johnson, Striley, \& Cottler, 2006).

Johnson and colleagues (2006) recently found that drug use was a precipitant to a traumatic event in a sample of out-of-treatment African American drug users $(N=1098)$, with $16 \%$ of the sample with trauma and poly-drug use reporting that the onset of all drug use (with the exception of crack cocaine) occurred prior to the trauma. In a longitudinal investigation examining the association between intimate partner violence and SUDs among women $(N=724)$ ages 18 to 30 , both "hard" drug use and marijuana use were related with an increased risk of experiencing violence in new relationships (Testa, Livingston, \& Leonard, 2003). Cottler and colleagues (1992) also found that physical assault often triggered PTSD among cocaine abusers, and that drug use preceded the onset of PTSD symptomatology. Furthermore, their study showed that opiate and 
cocaine users were over three times more likely to endure a physical assault than the nondrug using comparison subjects matched on traumatic experiences, and were more likely than the comparison subjects to develop PTSD as a result of these events (Cottler, Compton, Mager, Spitznagel, \& Janca, 1992). Finally, a significant number of women with SUDs are involved in intimate relationships with other drug and alcohol abusers, and drug problems of the abusive partner may play a role in both violence and in the woman's own drug abuse problems (Giles-Sims, 1998).

\section{Third Factor Theory}

It has also been suggested that overlapping genetic and environmental influences are possible contributing factors to the common etiology of SUDs and PTSD (Brown \& Wolfe, 1994; Xian, et al., 2000). In a review of co-occurring SUDs and PTSD, Brown and Wolfe (1994) concluded that SUDs and PTSD are multifactorial in etiology, and that in order to understand this co-occurrence, it is necessary to take into account biological, environmental, and psychological factors. Research using family, adoption, and twin samples identify a genetic influence on alcohol dependence (Kendler, et al., 1997; Pickens, et al., 1991), drug dependence (Grove, et al., 1990; Pickens, et al., 1991), and trauma exposure and PTSD (Jang, et al., 2003; Reich, et al., 1996; Stein, et al., 2003).

Recently, Xian and colleagues (2000) published a study that investigated the degree to which environmental and genetic influences contributed to the lifetime cooccurrence of SUDs and PTSD in a study of monozygotic and dizygotic male-male twin pairs members $(N=3304)$ who were members of the Vietnam Era Twin Registry. Using a genetic model fitting, Xian and colleagues (2000) found that SUDs and PTSD had 
etiologically distinct components as well as significant genetic and specific environmental contributions in common. Specifically, they found that the liability for PTSD was partly owing to a $15.3 \%$ genetic contribution common to drug and alcohol dependence and $20.0 \%$ genetic contribution specific to PTSD. They also found that having a risk for alcohol dependence was partly due to a $55.7 \%$ genetic contribution that is common to drug dependence and PTSD. Furthermore, genetic influences common to PTSD and alcohol dependence constituted $25.2 \%$ of the total risk for drug dependence, and certain family environmental influences accounted for $33.9 \%$ of the total variance in drug dependence risk.

\section{Implications of Co-Occurring Trauma and SUDs}

\section{Trauma and Health among Women with SUDs}

While SUDs are associated with a wide range of physical and mental health detriments, PTSD confers an even greater risk and detriment. Women with SUDs who have also experienced severe trauma have disproportionate physical health burdens, and are especially susceptible to risk factors related to poor health and disability (Larson, et al., 2005; Reynolds, et al., 2005). These women generally report significantly more chronic physical symptoms, poorer health perceptions, and a higher rate of neurological and cardiovascular symptoms compared to those without PTSD (Ouimette, Goodwin, \& Brown, 2006).

For instance, Larson and colleagues (2005) found that almost half (48\%) of the women in a cross-site study of specialized services for women with histories of trauma and co-occurring SUDs $(N=2,729)$ reported serious physical illnesses that frequently 
restricted their daily life activities or required the use of special equipment. Health status was rated as poor or fair among $46 \%$ of these women. Ouimette and colleagues (2006) recently examined the effects of PTSD on health and well being among patients with SUDs and found greater cardiovascular and neurological symptoms in SUD patients with PTSD compared to those who did not have PTSD, even after controlling for ethnicity and gender. SUD patients with comorbid PTSD also reported worse bodily pain and a greater number of chronic physical symptoms as well as poorer scores on general health as compared to SUD patients without PTSD (Ouimette, Goodwin, \& Brown, 2006).

Women with trauma histories are also more likely than women without trauma to be hospitalized with both trauma and non-trauma related medical problems. For instance, in study examining childhood abuse and domestic violence among women in methadone maintenance treatment $(N=151)$, results indicated that a greater proportion of abused women than non-abused women reported being admitted to a hospital within the past three months and having had a major illness within the past three months (Gilbert, Bassel, Shilling \& Friedman, 1997).

The proposed study will assess the frequency of common physical symptoms and sensations, and expects to find that SUD women with PTSD symptomatology will experience greater health problems than SUD women without PTSD symptomatology, supporting the need for an intervention that may help improve health outcomes among SUD patients. 


\section{Clinical Profiles among Women with SUDs and Trauma}

In addition to health problems, studies consistently reveal that women with comorbid SUD and PTSD are likely to have greater addiction severity, higher rates of interpersonal and psychological problems, additional psychiatric disorders, lower global levels of functioning, a lower motivation for drug treatment, and poorer treatment outcomes than those without PTSD or significant trauma histories (Clark, Masson, Delucchi, Hall, \& Sees, 2001; Coffey, Schumacher, Brimo, \& Brady, 2005; Najavits, Weiss, \& Shaw, 1997).

For instance, among opiate dependent women in methadone treatment $(N=150)$, Clark and colleagues (2001) found that violent trauma and distress was highly correlated with severity of drug use (as measured by the Addiction Severity Index [ASI]). Furthermore, the use of "harder" drugs, such as cocaine and heroin, are consistently found to show a stronger correlation with trauma and/or a PTSD diagnosis than other drugs, such as alcohol or marijuana (Grice, et al., 1995; Miller, Downs, \& Testa, 1993). Women with comorbid SUDs and PTSD are also at increased risk for a range of other problems, including interpersonal deficits, custody battles and mistreatment of their children, HIV risk, homelessness, and domestic abuse (Brady, Dansky, Sonne, \& Saladin, 1998; Hien, Cohen, \& Campbell, 2005).

In a study comparing women $(N=57)$ with SUD and PTSD (dual diagnosis group; $n=28$ ) to women with PTSD only (single diagnosis group; $n=29$ ), Najavits and colleagues (1999) found more severe clinical profiles and interpersonal problems among the dually diagnosed individuals as compared to the other group. Women with SUD- 
PTSD reported: worse life circumstances since childhood (e.g., fewer opportunities in life, less attractive physical appearance); more criminal behavior; more siblings with drug problems (50.0\% versus $20.7 \%$ ); and greater lifetime suicide attempts (78.6\% versus $51.7 \%)$. Virtually all of the women (98\%) with SUD-PTSD reported experiencing their first trauma prior to 18 years of age. Furthermore, PTSD preceded SUD onset for the majority of women (61\%), offering support for the self-medication hypothesis (Najavits, Weiss, \& Shaw, 1999).

Women with a history of PTSD are also more likely than those without PTSD to be diagnosed with depression and dysthymia, to report higher rates of depressive symptoms, and are more likely to have attempted suicide (Clark, et al., 2001). Among inner-city, African American women, depressive symptoms are found to be greater among women who report both high levels of intimate partner violence and alcohol problems (Paranjape, et al., 2007). Cross-sectional studies reveal that females with comorbid SUD-PTSD have significantly worse clinical profiles than females with only one of the two disorders (Brown, Stout, \& Mueller, 1999; Najavits, Weiss, \& Shaw, 1999; Ouimette, Wolfe, \& Chrestman, 1996). Women with SUDs and current PTSD, compared to those without, have significantly higher rates of medical problems, interpersonal problems, show greater resistance to treatment, and have higher rates of cooccurring axis I and axis II disorders, as well as higher levels of psychopathological symptomatology (Najavits, et al., 1999).

In a well designed study that used both self-report and interview measures of psychopathology and substance use with cocaine-dependent outpatients $(N=122)$, those 
with a comorbid PTSD diagnosis had a greater number of axis I disorders (e.g., Major Depression)on the Structured Clinical Interview for DSM-III-R (SCID), axis II disorders (e.g., greater proportion of obsessive-compulsive personality disorder) on the SCID, Beck Depression Inventory scores $(M=15.8$ for those with PTSD versus $M=7.3$ for those without comorbid PTSD), 17-item Hamilton Depression scale scores ( $M=12.4$ versus $M$ = 5.5), the Brief Symptom Inventory total score $(M=1.14$ versus $M=0.37)$, the Beck Anxiety Inventory scores $(M=12.5$ versus $M=4.2)$, and the Inventory of Interpersonal Problems score ( $M=1.47$ versus $M=0.98)$ (Najavits, Gastfriend, et al., 1998). Other research indicates that compared with non-PTSD SUD female patients, SUD-PTSD female patients have a greater likelihood of meeting diagnostic criteria for major depressive disorder, panic disorder, bipolar disorder, and Cluster B personality disorders (Brown, Stout, \& Mueller, 1999).

Ouimette, Wolfe, and Chrestman (1996) compared characteristics of women $(N=$ 47) diagnosed with PTSD and alcohol abuse (i.e., PTSD-AA; $n=12$ ), women with PTSD-only ( $n=13)$, and women with neither alcohol abuse nor PTSD (i.e., controls; $n=$ 22), using a sample of women who had served overseas during the Vietnam era (19641975). Findings revealed that the PTSD-AA group, compared to the PTSD-only group and the control group, reported more childhood sexual abuse experiences, a greater number of traumatic events, and a more severe history of adult sexual assault. In addition, sexual victimization specific to wartime differentiated those with PTSD-AA from the other groups, suggesting that sexual victimization occurring during wartime may have played a role in later alcohol abuse (22 years old was the mean age of entry into 
military, while 26 years old was the average age of onset for alcohol abuse/dependence). Furthermore, women with PTSD-AA suffered from a greater range of symptoms (e.g., borderline traits, dissociation, and more severe PTSD symptomatology) than women with PTSD-only or controls. Women with PTSD-AA also reported poorer affect regulation, impulsivity, and difficulties with interpersonal relationships (Ouimette, Wolfe, \& Chrestman, 1996).

Numerous studies have indicated that women with co-occurring PTSD-related symptomatology and SUD have worse compliance with aftercare for SUD treatment and more addiction-related problems (e.g., physical appearance, criminal behavior, etc.) (Brady, Killeen, Saladin, Dansky, \& Becker, 1994; Gutierres \& Van Puymbroeck, 2006; Najavits, Weiss, \& Shaw, 1999). The proposed study expects to find higher levels of psychological distress and increased rates of symptoms of depression among female SUD residential patients who have experienced trauma compared to those without trauma, emphasizing the importance of prevention and intervention efforts.

\section{Importance of Addressing Trauma in SUD Treatment}

Comorbid PTSD is correlated with low rates of treatment engagement and a high incidence of treatment drop-out among both women and men with SUDs (Brown, Recupero \& Stout, 1995; Ouimette, Finney \& Moos, 1999). These individuals tend to have less effective coping strategies, including fewer expected benefits from quitting, greater use of emotional discharge coping (e.g., yelling, risk taking), and more positive expectancies about substance use (Ouimette, Ahrens, Moos \& Finney, 1997). Chronic substance use may reduce emotional reactions related to traumatic experiences, thus 
reinforcing the potential of future drug use (Stasiewicz \& Maisto, 1993). Negative emotions related to trauma may increase substance craving, and can contribute to generally poorer treatment outcomes (Coffey, et al., 2002). Thus, studies suggest that treatments that diminish negative emotions related to trauma may also decrease craving, which could contribute to better treatment outcomes (Coffey, et al., 2002).

Since untreated PTSD symptomatology may contribute to poorer treatment outcomes, the association between trauma and SUDs may be relevant for SUD treatment (Brady, Killeen, Saladin, Dansky, \& Becker, 1994). Improving severe drug abuse may uncover PTSD-related symptoms and heighten subjective distress, and untreated PTSDrelated symptoms may contribute to relapse to substance use (Clark, et al., 2001). While some untreated PTSD symptoms improve (e.g., global measures of intensity/frequency) with cessation of drug use, other symptoms of PTSD (e.g., intrusive thoughts, nightmares, flashbacks) are unlikely to improve with SUD treatment alone (Clark, et al., 2001; Dansky, Brady, \& Saladin, 1998). Additionally, research indicates that while remission from PTSD is associated with better SUD outcomes, remission from SUD is not associated with improved PTSD outcomes (Ouimette, Brown, \& Najavits, 1998), indicating that in SUD treatment settings, approaches that specifically target trauma may be necessary if PTSD symptoms are expected to improve (Dansky, Brady, \& Saladin, 1998).

While significant experiential avoidance is present in both PTSD and SUDs (Morrisey, et al., 2005), patients with both problems are likely to get substance abuse services or mental health services, but not both (Batten \& Hayes, 2005). Experiential 
avoidance is defined as purposeful, deliberate attempts to change the frequency or form of selective private events, such as unwanted thoughts, images, memories, emotions, and bodily sensations, or the circumstances in which they occur (Zettle, Petersen, Hocker, \& Provines, 2007). In many SUD treatment settings, trauma and PTSD are rarely assessed and trauma-focused interventions are not part of treatment services (Ouimette, Moos, \& Finney, 2003; Reynolds, et al., 2005). The patients' own numbing and embarrassment about trauma may work against trauma symptom recognition (Brown, Recupero, Stout, 1995). Also, both drug use and drug withdrawal may intensify, imitate, or complicate symptoms of PTSD, making identification more challenging (Reynolds, et al., 2005).

Historically, there has been an ongoing debate regarding which problem to treat first; PTSD or SUDs. Among SUD treatment providers, the general consensus had been that addressing trauma during the early phase of SUD recovery may "open Pandora's box" and derail SUD treatment improvement (Hien, Cohen, Miele, Litt, Capstick, 2004). There is now evidence, however, to suggest that trauma should be treated, even in the earliest stages of SUD treatment. While requiring drug use cessation before trauma treatment may aid in the implementation of current trauma interventions, it may do so at a cost for those with dual problems (Batten \& Hayes, 2005). Research strongly advocates that counselors address both problems simultaneously, with evidence that such an approach actually contributes to addiction recovery, rather than derailing SUD patients from abstinence (Brady, Dansky, Back, Foa, Carroll, 2001; Hien, et al., 2004; Najavits, 2006). 
Despite such research findings, many drug treatment programs continue to ignore trauma and PTSD, suggesting that more education and training are needed. The proposed study expects that a brief intervention, which addresses traumatic/stressful experiences among women in SUD treatment, may improve treatment outcomes, such as posttraumatic symptom severity, health symptoms, depressive symptoms, drug cravings, and psychological distress related to the emotional experience. In addition, if effective, the proposed intervention requires little training and minimal costs, making it readily transferable from research to clinical practice

Treatment for patients with co-occurring SUD and PTSD symptomatology is an important issue, not only because of the frequency and severity of co-occurring SUD and PTSD, but also because treatments traditionally used for treating one of these problems may be inadequate to treat the combination of both problems. For instance, benzodiazepines, which are commonly used for the treatment of anxiety disorders, are commonly contraindicated for women who are prone to addiction (Najavits, Weiss, \& Shaw, 1997). On the other hand, twelve-step self-help groups such as Alcoholics Anonymous (AA), which are often effective for individuals with SUDs, may be less applicable for women with comorbid PTSD-SUD, particularly early in their recovery because the groups: often focus on the past (which is contraindicated in many traumafocused treatments); are frequently attended by both males and females; and require individuals to share their stories publicly as they "surrender to a higher power" (Najavits, et al., 1997). 
Issues of treatment for women with co-occurring SUD and PTSD are heightened by clinical observations revealing that these women have a propensity for inducing uneasy alliances, a powerful negative countertransference by therapists, numerous crises, and are often labeled with a "poor prognosis" (Najavits, et al., 1997). The proposed study expects that a brief intervention, which requires little training and minimal costs and addresses traumatic/stressful experiences among women in SUD treatment, may improve treatment outcomes and distress related to the emotional experience.

\section{Assessing Trauma in SUD Treatment}

While there is overwhelming empirical evidence suggesting significant comorbidity of SUD and PTSD, unfortunately SUD treatment providers frequently fail to notice trauma and PTSD in their patients (Coffey, Schumacher, Brimo, \& Brady, 2005). Treatment providers should be mindful that women do not readily recognize trauma or victimization as adults or children during intake interviews (Gutierres \& Van Puymbroeck, 2006). In addition, the timing of assessments (i.e., when reliable and valid information is most likely to be obtained) and methods for assessment (e.g., interviews versus questionnaires) are important issues to consider (Read, Bollinger, \& Sharkansky, 2003).

A key issue in the assessment procedures is that any drug use by individuals could mask or diminish PTSD symptomatology. Thus, PTSD assessment should not be done while the person is actively using drugs and/or alcohol. Assessment of trauma and PTSD symptoms should preferably occur after withdrawal symptoms have abated (Read, Bollinger, \& Sharkansky, 2003; Saladin, Brady, Dansky, \& Kilpatrick, 1995). Since 
memory difficulties can be related with drug withdrawal, assessment of traumatic experiences and associated consequences will be more dependable following detoxification. Also, providers should be aware that withdrawal symptoms from drugs and alcohol (e.g., nightmares, increased anxiety, and difficulty sleeping) may overlap with symptoms found in PTSD, which can easily skew estimated rates of PTSD, and make it more challenging to reliably identify co-occurring conditions (Brown, et al., 1999; Read, et al., 2003). Thus, treatment providers should check for potentially separate and complicating problems, because without such diagnostic assessments and consequent treatment recommendations and referrals, PTSD and trauma patients may not get sufficient care, which could eventually lead to poorer treatment outcomes (Brown, et al., 1999).

There are several self-report instruments that provide an effective and timeefficient method of assessing trauma and PTSD. These instruments evaluate traumatic exposure and incorporate DSM-IV criteria, and can be given in a variety of research and clinical settings. Self report questionnaires offering the most promise in facilitating the recognition of trauma and PTSD, specifically with respect to co-occurring SUD-PTSD populations include: the PTSD Symptom Scale-Self Report (PSS-SR; Foa, Riggs, Dancu, \& Rothbaum, 1993), the PTSD Checklist (Weathers, Litz, Herman, Huska, \& Keane, 1993), and Posttraumatic Stress Diagnostic Scale (PDS; Foa, 1996). All of these measures have strong psychometric properties with both treatment-seeking and community samples (Read, Bollinger, \& Sharkansky, 2003). 


\section{Expressive Writing}

\section{Expressive Writing and Disclosure}

Accumulating evidence over the past two decades suggests that disclosure of traumatic and emotional experiences through expressive writing has widespread physical and mental health benefits (Pennebaker \& Seagal, 1999). The typical laboratory writing technique involves randomly assigning individuals to one of two or more writing groups. All groups are instructed to write about assigned topics for 15 to 20 minutes a day over three to five consecutive days, and the writing is typically done with no feedback given. Individuals who are assigned to the expressive writing group are asked to write about the most traumatic or stressful event of their lives, while those who are in the control conditions are generally instructed to write about superficial topics, such as how they use their time (Pennebaker, 1997; Sloan \& Marx, 2004).

Beneficial effects related to writing about emotional experiences have been shown in areas as varied as health, achievement, and general well-being (Slatcher \& Pennebaker, 2006). For instance, studies using Pennebaker's expressive writing paradigm reveal that writing about a highly stressful or traumatic event is associated with improved immune functioning (Booth, Petrie, \& Pennebaker, 1997), a decrease in health center and doctor's visits (Pennebaker, Kiecolt-Glaser, \& Glaser, 1988; Pennebaker, Mayne, \& Francis, 1997), fewer missed days of work or school (Francis \& Pennebaker, 1992), decreases in sympathetic nervous system activity (McGuire, Greenberg, \& Gervirtz, 2005; Pennebaker, Barger, \& Tiebout, 1989; Pennebaker, Hughes, \& O'Heeron, 1987), and a 
reduction in the degree to which the experience is distressing or painful (Lepore \& Smyth, 2002).

Pennebaker and Beall (1986) found that expressive writing (writing about an earlier trauma) was related with short-term heightened physiological arousal and longterm decreases in health problems among undergraduate students $(N=46)$. In an examination of autonomic effects of expressive writing in people with elevated blood pressure $(N=38)$, systolic and diastolic blood pressure was significantly decreased among those in the expressive writing group as compared to the control writing group (McGuire, Greenberg, \& Gevirtz, 2005). McGuire and colleagues (2005) also found a significant interaction revealing that the very low frequency of heart rate variability rose over time in the control writing group only, indicating a possible buffering effect in expressive writing. In another study, university employees $(N=41)$ who wrote about a traumatic experience showed a significant reduction in absentee rates from work $(28.6 \%$ reduction) while employees who wrote about a non-traumatic topic showed an increase in absentee rates ( $48.5 \%$ increase) during the month of writing relative to the eight months prior to the experiment (Francis \& Pennebaker, 1992). In a study looking at the effects of expressive writing on PTSD symptoms and depression among women who have endured intimate partner violence $(N=47)$, those who were depressed showed a significantly greater drop in depression if they were in the traumatic disclosure (experimental) writing group as compared to the neutral topic (control) group (Koopman, et al., 2005).

In a meta-analysis of expressive writing studies $(N=13$ studies that used randomized experiments) the included data from over 800 participants, Smyth (1998) 
found a $23 \%$ symptom improvement in the traumatic disclosure writing condition over the control writing condition, which indicates a moderate effect size $(d=.47 ; r=.23, p$ $<.0001)$. This effect size is comparable or larger than those found in other psychological, educational, or behavioral interventions (Pennebaker \& Chung, in press; Smyth, 1998). For instance, in a review of approximately 500 studies on psychotherapy, Smith and Glass (1977) found an average 32\% greater improvement among those receiving psychotherapy compared to those who did not.

Another recent meta-analysis examining experimental disclosure and its moderators, using 146 randomized studies (48\% unpublished), found experimental disclosure to be effective, with an average effect size of $r=.075$ (Frattaroli, 2006). Results showed that individuals in expressive writing conditions revealed improved psychological health, physiological functioning, disease specific outcomes and illness behaviors, and general functioning (Frattaroli, 2006).

Thus, expressive writings' efficacy, efficiency (15-20 minutes per session over three to seven consecutive days, compared to psychotherapy, which usually occurs for an hour per week over several months or years), and cost-effectiveness (the cost of a notebook), suggest that writing as a means for disclosing traumatic experiences may be a powerful adjunct to traditional alcohol and drug treatment, particularly in SUD treatment settings that do not routinely screen for trauma and PTSD and therefore, do not specifically focus on such issues during treatment. 


\section{Expressive Writing with Different Populations}

Early studies of expressive writing were conducted mainly with university students (Frattaroli, 2006). However, more recently, the effects of written disclosure have been studied in more varied samples. Studies using non-student samples, for example, found that expressive writing was beneficial in decreasing trips to the infirmary among male prison inmates (Richards, Beal, Seagal, \& Pennebaker, 2000), and in helping recently unemployed professionals find employment faster (Spera, Buhrfeind, \& Pennebaker, 1994).

Studies suggest the disclosure paradigm is as beneficial for maximum security prisoners with $6^{\text {th }}$ grade educations as it is for senior professionals with advanced degrees (Richards, et al., 2000; Spera, et al., 1994), and yields positive outcomes regardless of ethnicity or native language among college students (Pennebaker \& Chung, in press). For example, Richards and colleagues (2000) examined expressive writing on illness behavior among psychiatric prison inmates $(N=98)$ and found a significant decrease in infirmary visits among sex offenders in the trauma writing condition. The prisoners had a sixth grade level education or higher, with a mean of 11.9 years of education completed. In an examination of expressive writing and coping with job loss among recently unemployed professionals $(N=63)$ who had an average tenure of 20 years of employment with advanced degrees, the expressive writing condition (writing about their deepest thoughts and feelings surrounding job loss) was associated with being reemployed more quickly than both the non-traumatic writing condition and the nonwriting condition (Spera, et al., 1994). Expressive writing appeared to influence the 
person's attitude with regard to their old job and finding new employment. Interestingly, while the study was originally slated to last one year, at four months authors discovered that those in the expressive writing condition were being rehired at a significantly higher rate than those in either control conditions, and therefore terminated the study early in order to invite the people from the control conditions to receive the expressive writing condition.

The expressive writing paradigm has also recently been expanded to include individuals with medical ailments, such as rheumatoid arthritis, asthma, migraines (Smyth, Stone, Hurewitz, \& Kaell, 1999), chronic pelvic pain (Norman, Lumley, Dooley, \& Diamond, 2004), and breast cancer (Low, Stanton, \& Danoff-burg, 2006). Patients in the written disclosure conditions show clinically relevant improvements in symptomatology and health status relative to patients who do not write (Smyth, et al., 1999).

For instance, a study of patients $(N=107)$ with asthma $(n=49)$ or rheumatoid arthritis $(n=49)$ found that asthma patients who were in the experimental condition $(n=$ 39) and wrote about the most stressful event of their lives for 3 consecutive days, showed improvements in lung function at four month follow-up, whereas asthma patients who were in the control group $(n=22)$, and wrote about emotionally neutral topics for 3 consecutive days, showed no change at four month follow-up (Smyth, et al., 1999). A similar intervention was done with rheumatoid arthritis patients, and Smyth and colleagues (1999) found that those in the experimental group $(n=32)$ showed improvements in overall disease activity at the four month follow-up, whereas control 
group patients $(n=19)$ did not change. Smyth and colleagues (1999) reported that after combining all patients, almost half (47.1\%) of experimental patients showed clinically relevant improvement, whereas less than one quarter (24.3\%) of control patients showed improvement.

Likewise, in an examination of expressive writing with breast cancer patients $(N=$ 60), written disclosure was found to produce significantly reduced physical symptoms and medical appointments for cancer-related morbidities among breast cancer patients relative to patients in a fact-writing control condition (Stanton, Danoff-Burg, \& Sworowski, 2002). In another study looking at women with chronic pain $(N=48)$, Norman and colleagues (2004) found that compared with control writing, women in the disclosure writing group had less disability, particularly when the women had higher ambivalence scores at baseline.

\section{Mechanisms through which Expressive Writing Works}

While expressive writing is shown to be beneficial, the underlying mechanisms through which it works are not well understood (Sloan \& Marx, 2004). A few of the most widely used explanatory mechanisms that have been suggested to account for the observed benefits include the inhibition theory (Pennebaker, 1989), cognitive processing theory (Pennebaker, 1993), and self-regulation theory (Greenberg, et al., 1996; Lepore, Greenberg, Bruno, \& Smyth, 2002).

Inhibition Theory. The inhibition theory argues that inhibition of feelings and thoughts concerning an upsetting experience is detrimental; therefore, disclosure of feelings and thoughts can diminish threats and enhance various psychological and 
physical health outcomes (Pennebaker, Barger, \& Tiebout, 1989; Pennebaker, Hughes, \& O'Heeron, 1987). Inhibition may partially mediate the association between the psychological threat and illness. Specifically, physiological work of inhibition (i.e., physiological exhaustion), the interruption of cognitive processing prohibiting resolution, and the heightened social isolation of not disclosing significant matters to others, can all add to stress and illness (Pennebaker \& Keough, 1999).

Inhibition of the traumas can emerge through dreams, ruminations, intrusive memories, and related behaviors and cognitive symptoms. By inhibiting, people may not interpret the event into a language that would allow for understanding and assimilation of the experience (Pennebaker, 1997; Pennebaker \& Keough, 1999). Yet, since it is not always necessary for traumatic experiences to be previously undisclosed, studies suggests an inhibition explanation may not be fully adequate to explain benefits of expressive writing (Greenberg \& Stone, 1992).

Cognitive Processing Theory. The cognitive processing theory (Pennebaker, 1993) suggests that confronting a traumatic event through expressive writing may help to organize, assimilate, give meaning, or incorporate the upsetting event into one's selfschema (Pennebaker \& Beall, 1986). Thus, expressive writing may allow individuals to gain insight into what happened. Merely expressing trauma is not sufficient, but rather health benefits appear to involve translating events into language (Pennebaker, 1997).

Three linguistic factors: increased positive emotion words, a moderate number of negative emotion words, and, most important, an increased use of causation words (e.g., because, cause, effect) and insight words (e.g., consider, know) over the course of 
writing, appear to be significantly related with benefits and improved health (Pennebaker, 1993; Pennebaker \& Keough, 1999; Pennebaker, et al., 1997). Also, individuals who start with poorly organized accounts and progress to coherent stories by the last day of writing benefit significantly from expressive writing (Pennebaker, 1997). The proposed study will examine written essay content via a computerized text-analysis program that calculates words of multiple categories (Linguistic Inquiry and Word Count [LIWC]; Pennebaker, Francis, \& Booth, 2001).

Self-Regulation Theory. The self-regulation or emotion-regulation theory posits that self-regulation may mediate the beneficial effects of expressive writing (Lepore, Greenberg, Bruno \& Smyth, 2002). Lepore and colleagues (2002) suggest that experimental disclosure may help by allowing people to observe themselves expressing and controlling their emotions. Thus, written disclosure may offer a new or stronger sense of self-efficacy or self-regulation for the individual (Frattaroli, 2006).

After emotional disclosure, one may feel that their traumatic experience(s), stressors, or challenges are more controllable, which may help diminish negative affect and contribute to other improvements in overall well-being (Lepore, Greenberg, et al., 2002). Written disclosure through expressive writing can help an individual comprehend or acknowledge his or her response to the event, make sense of the event, explore sources of emotions, clarify goals, and habituate to the traumatic event through written exposure.

In accordance with the above theories of expressive writing, many trauma theorists maintain that the recovery process from trauma entails special processing of traumatic memories: skills to organize, articulate, and streamline the traumatic event has 
been recommended as markers to recovery. Observations made in clinical settings indicate that trauma narratives told by individuals with chronic PTSD are characterized by incomplete sentences and remarks that reveal confusion (Foa \& Street, 2001).

Furthermore, research on trauma and PTSD suggest that the extent to which the individual (a) organizes and articulates the traumatic memory; (b) is emotionally engaged with the traumatic memory; and (c) upholds a balanced view about the world and self influences whether that individual will improve or develop chronic disturbances (Foa \& Street, 2001). 
Statement of the Problem and Hypotheses

Women with SUDs are identified as high risk for co-occurring traumatic stress exposure. As many as $80 \%$ of women needing treatment for drug problems report lifetime histories of physical and/or sexual assault (Hien, Cohen, \& Campbell, 2005), and many display Posttraumatic Stress Disorder (PTSD) symptomatology (e.g., Brown, Stout, \& Mueller, 1999). In these settings, $55 \%$ to $99 \%$ of women report at least one lifetime trauma (Najavits, Weiss, \& Shaw, 1997). Trauma and addiction comorbidity studies consistently reveal these women are likely to have poorer health and increased disability, more severe clinical profiles, and worse treatment adherence, than those without traumatic experiences or PTSD (Najavits, 2006).

The proposed investigation seeks to examine whether Pennebaker's expressive writing paradigm is beneficial for these women, who are currently undergoing residential SUD treatment. The expressive writing paradigm includes writing about an assigned topic for 15-20 minute sessions per day over three to five consecutive days. Past studies have demonstrated the efficacy of expressive writing with significant reductions in distress (Smyth, 1998), and improved physical (e.g., Pennebaker, et al., 1997) and psychological health (Lepore \& Smyth, 2002). Its usefulness as an intervention for SUD populations is unknown, however. Historically, trauma has not been incorporated into SUD treatment programs; however, studies indicate that addressing trauma during SUD treatment may improve treatment (e.g., Hien, et al., 2004). 
The proposed investigation seeks to examine whether Pennebaker's expressive writing paradigm is beneficial for women currently undergoing SUD treatment. If expressive writing improves outcomes for women with SUDs, these findings would support its use as a brief, cost effective, adjunct to current SUD treatment.

The specific aims of this study are to: 1) investigate physical and mental health problems, as well as the relationships between PTSD and/or trauma and physical and mental health problems; 2) compare outcomes for a treatment-based sample of substance abuse/dependent women assigned to either a expressive writing condition (emotional topic) or a control writing condition (neutral topic); and 3) examine the influence of the writing task on both immediate and longer-term levels of psychological distress.

Based on previous literature, it was hypothesized that: 1) women with co-morbid PTSD and/or significant trauma will have more severe psychological and physical health problems at baseline; 2) at two weeks and one month following the 4-day writing intervention, participants randomized to the expressive writing condition will show greater reductions in post-traumatic symptom severity, health symptoms, psychological distress, and depressive symptoms, than participants randomized to the control writing condition; and that 3) women randomized to the expressive writing group would show higher levels of immediate distress (measured immediately after each writing session) and lower levels of longer-term distress (measured at two weeks and one month postbaseline assessment) relative to those in the control group. 
Methods

Objectives of Proposed Study

The primary goal of this study is to test whether Pennebaker's expressive writing paradigm is a useful therapeutic adjunct for women in residential treatment for SUDs.

This study was approved by Virginia Commonwealth University's Institutional Review Board under "Expressive Writing as a Therapeutic Process for Drug Dependent Women," protocol number HM10881.

Study Site

The study occurred at Rubicon, Inc., a large nonprofit organization located in Richmond, Virginia, that provides residential treatment services to women with SUDs. Specific services at Rubicon include individual and group counseling, motivational enhancement therapy groups, and case management for such needs as housing, transportation, and childcare.

Experimental Overview

Women enrolled in residential substance abuse treatment at Rubicon, Inc. were invited to participate in a randomized clinical trial comparing expressive writing to a control group. The study involved baseline assessment, followed by 20 minute daily writing sessions that took place over four consecutive days. For the experimental (expressive writing) group, writing sessions focused on life experiences while the control group participants wrote about neutral topics. Follow-up assessments occurred at 2 
weeks and 1 month post-intervention and focused on psychological functioning, drug craving and other relevant domains of psychosocial functioning.

At baseline, participants who provided informed consent completed questionnaires assessing demographic information, post-traumatic severity, distress, craving, depressive, drug use, and health symptoms. Group assignment occurred prior to the first writing session, with participants randomly assigned to either the experimental or control writing groups. Those in the experimental (expressive writing) group were asked during writing sessions $1-4$, to write about personal traumatic/stressful experiences, while those in the control writing group were asked to write on neutral topics (e.g., what they ate on the previous day, what they did since waking up yesterday). At 2 week and 1 month post-intervention visits, participants were asked to complete a follow-up packet consisting of the same questionnaires administered at baseline (except the demographic questionnaire and the SCID), as well as a specific follow-up questionnaire that included questions about what participants thought of the writing, while also acting as a manipulation check to find out if participants heard about the other writing instructions. Participants

Participants were recruited in-person by the lead investigator or another experimenter, who was unaffiliated with Rubicon's treatment. Women were approached within the first few days of residential treatment and asked to volunteer for a study in which they will be asked to write stories related to their life (Sloan \& Marx, 2004).

Recruitment. Recruitment occurred at the Rubicon, Inc., a residential women's substance abuse treatment facility from June 17, 2007 through November 06, 2008. 
Program admission records were used to identify women who had recently been admitted for residential care. Once the principal investigator (PI) or other research staff members identified a new admission, the woman was asked to report to the Staff on Duty (SOD) office. Care was taken to minimize disruption in the woman's standard program activities. When the patient arrived at the SOD office, she was asked about her projected length of stay at Rubicon (which typically varies from 30 to 60 days, but could be extended if warranted). If the patient had been approved to receive at least 60 days of residential care, the PI or other research staff would use the following recruitment script (approved by VCU's IRB):

I would like to let you know about a research study being conducted by researchers from Virginia Commonwealth University (VCU) that involves women being treated for substance abuse. The purpose of this research is to study women's reactions to journal writing. It will take about 10 minutes of your time to hear more about it. If you qualify, you could earn up to $\$ 50.00$ in gift cards for your time and effort for participating in the study. Your decision to be in the study or not will not impact your treatment here at Rubicon. The study is totally separate, and your decision to participate in this research is completely voluntary.

If the patient was interested in participating, informed consent was obtained and the participant was provided with a copy of the consent document, while the original consent form was retained by the research team and filed in a locked drawer. 
Inclusion criteria: To be included in the study, a woman had to: 1) Be at least 18 years of age; 2) Meet DSM-IV criteria for a SUD; and 3) Have approval for 60 days of residential treatment at Rubicon.

Exclusion criteria: Individuals were ineligible for study participation if they: 1) Had an acute or chronic mental disorder that would make them unable to follow study protocol procedures (e.g., chronic psychosis, mental retardation, current suicidality); or 2) Had problems that would prevent them from completing the writing intervention, the baseline assessment, or the follow-up assessment (e.g., illiteracy). To assess for literacy, participant will be asked to read a part of the consent form aloud.

Risk for Recruitment Bias. It should be noted that from 10/17/08 through 07/16/08, another study by other members of our research team was recruiting subjects concurrent with the present study. The other research project focused on women with alcohol problems and investigators targeted women approved for 30 as well as 60 days of treatment. To determine the impact this might have on sample representativeness, the number of women referred to the other project was tracked throughout the recruitment period. Specifically, $n=17$ women were recommended for the other study and excluded from the present research. These $n=17$ women were found to be eligible for the present study, but were considered ineligible because they were already enrolled in the other project (which began before the present study started to enroll participants).

Sample Size. A power analysis using 0.80 power, an alpha level of 0.05 , and a written disclosure effect size of 0.23 , indicates that a minimum of 30 participants per condition would be sufficient to find a medium effect, requiring a minimum total sample 
size of 60 women (Cohen, et al., 2003) to achieve primary study outcomes. An additional 90 women, which will increase the sample size to 150 participants, were recruited and included in order to allow for subsequent analyses for such things as patient race, degree of substance abuse, and trauma severity. The present study was initially funded as a pilot study through the VCU Institute for Women's Health. Subsequently, a NIDA R36 Dissertation grant was awarded, which allowed the PI to increase sample size for the clinical trial. The increased sample size also provides enough power in order to examine subgroups within the sample for future analyses. Attrition rates in previous written disclosure studies range from 4\% (Sloan \& Marx, 2004b) to 13\% (Greenberg \& Stone, 1992). Assuming a $13 \%$ attrition rate, participants were recruited individually, and the protocol was administered individually until 150 women had been recruited for the study.

Study Procedures

\section{Baseline Assessment}

On the initial day of the study, participants were informed that the study would involve a baseline assessment (25 minutes), followed by four writing sessions over consecutive days, a two week follow-up assessment, and finally a one-month follow-up assessment, and that they could receive up to $\$ 50$ in gift cards if they participated in all phases of the research. The baseline assessment consisted of six measures (see below) and was administered in a confidential setting by the lead investigator or another experimenter. Study participants were assured that the data they provided would not be 
shared with Rubicon clinical/treatment staff and that they would receive a $\$ 5$ gift card after finishing the assessment battery.

Measures at Baseline. Participants were asked to provide basic demographic and personal information. The following measures were also administered:

The Posttraumatic Stress Diagnostic Scale (PDS; Foa, et al., 1997), a 49-item self-report measure, aids in PTSD diagnosis and symptom severity, with items that parallel to DSM-IV criteria (APA, 1994). The PDS has high test-retest reliability ( $r=$ $.83)$, high internal consistency $(r=.92)$, and high convergent validity (Foa, 1996).

The Brief Symptom Inventory (BSI; Derogatis, 1983), a 53-item inventory, is a shortened version of the SCL-90 (Symptom Check List-90) that assesses nine symptoms of distress and provides three global distress indices. The BSI has high scale-by-scale correlations with the SCL-90, high internal consistency (Cronbach's alpha: 0.71-0.85), convergent, discriminant, test retest reliability $(r=.68-.91)$, and construct validity (Derogatis \& Melisaratos, 1983). BSI indices include the following dimensions of distress: Somatization (SOM; distress arising from perception of bodily dysfunction); Obsessive-Compulsive (O-C; thoughts and actions experienced as unremitting and irresistible [e.g. checking and re-checking actions]); Interpersonal Sensitivity (I-S; feelings of personal inadequacy and inferiority); Depression (DEP; symptoms of the clinical depression); " Anxiety (ANX; symptoms associated anxiety); Hostility (HOS; thoughts, feelings, and actions of hostile behavior:); Phobic Anxiety (PHOB; symptoms of phobic anxiety or agoraphobia [e.g. fears towards public places, open spaces, travel, etc.]); Paranoid Ideation (PAR; characteristics including projection, suspiciousness, 
hostility, fear of loss of autonomy, and centrality); Psychoticism (PSY; mildly alien lifestyle at one extreme to floridly psychotic status at the other); and General Severity Index (GSI; depth of symptomatic distress experienced by the individual) (Derogatis \& Melisaratos, 1983).

The Center for Epidemiological Studies-Depression Scale (CES-D; Radloff, 1977), a 20-item self-report measure that assesses symptoms of depression, has high internal consistency for a psychiatric population $(\alpha .=.90)$.

The Pennebaker Inventory of Limbic Languidness (PILL; Pennebaker, 1982) is a 54-item scale that assesses the frequency of common physical symptoms and sensations. PILL has shown Chronbach's alphas ranging from .88 to .91, and 2-month test-retest reliabilities ranging from .79 to .83 .

The Structured Clinical Interview for DSM-IV-TR - Alcohol and Substance Use Disorders Module (SCID; First, et al., 2002) is a diagnostic interview assessing SUD diagnosis, including alcohol and other drugs. The SCID has demonstrated good validity and high interrater reliability for SUDs (Coffey, et al., 2002). The SCID was administered at baseline assessment only, and is and includes an interview component.

The Brief Substance Craving Scale (BSCS; Somoza, 1999) is a 12-item selfreport instrument assessing intensity, frequency, and length of craving over a 24 hour period for substances of abuse on a five-point Likert scale. Each of the three items related to the "intensity," "frequency," and "length" of craving during the prior 24 hours is answered on a 0-4 scale which was summed to yield a "total score" ranging from 0-12 (Somoza, et al., 1999). 
All measures are included in Appendix B, unless copyrighted.

\section{Expressive Writing Intervention}

Those who qualified for the study were randomized to either one of two conditions: control writing condition (neutral topic) or an expressive writing condition (emotional topic) using a random numbers table.

Writing Conditions: To be consistent with typical writing paradigm studies (Pennebaker \& Beall, 1986; Greenberg \& Stone, 1992), participants were asked to write over four consecutive days for 20 minutes per session. Writing instructions were adapted from instructions developed by Pennebaker (1997). All writing instructions were read out loud to the participant upon meeting with the investigator before each writing session. A copy of the exact writing instructions read by the investigator was also attached to the front flap of each journal so that the participant could re-read the instructions at any time.

Expressive Writing group. The standard writing instructions for those in the expressive writing condition adhered to the instructions used by Pennebaker \& Chung (in press):

Over the next four days, I want you to write about your deepest emotions and thoughts about the most traumatic experience in your life. In your writing, I'd like you to really let go and explore your very deepest emotions and thoughts. You might tie this trauma to your childhood, your relationship with others, including your parents, lovers, friends, or relatives. You may also link this event to your past, present, or your future, or to who you have been, who you would like to be, or who you are now. You may write about the same general issues or experiences on all days of writing or on different topics each day. Not 
everyone has had a single trauma but all of us have had major conflicts or stressors in our lives and you can write about them as well. All of your writing will be completely confidential. Don't worry about spelling, sentence structure, or grammar. The only rule is that once you begin writing, continue to do so until your time is up.

Control Writing group. Those in the control condition were asked to write about an assigned topic on each of the four writing days (description of what they had eaten since yesterday during the first session, how they spent their day during the second session, etc.). An example of the standard writing instructions for those in the control condition is as follows:

Over the next four days, I will give you a writing assignment. In today's writing, I would like you to describe what you have eaten since yesterday morning. I am not interested in your emotions or opinions. Rather I want you to write about specific objects or events in detail without discussing your thoughts or feelings. Feel free to be as detailed as possible. Again, I want you to describe exactly what you have eaten up until coming to this experiment. For example, you might describe the texture, the color, an estimate of calories and fat, how big the portions were, what meal you were eating, etc. The most important thing in your writing, however, is for you to describe what you ate since yesterday as accurately as possible.

Writing Session One. The day after baseline assessments were completed, participants were individually administered the appropriate writing instructions by the lead investigator or another experimenter. Writing instructions for each condition and session were based on the protocols used by Pennebaker, et al. (1988) and Sloan \& Marx 
(2004), and are outlined in Appendix A. Participants were asked not to discuss the study or the writing instructions they received with anybody while residing at Rubicon. After receiving the writing instructions from first experimenter, participants were introduced to a second experimenter (trained graduate student), who remained blind to the condition throughout the study. The second experimenter handed participants an immediate version of the Positive and Negative Affect Schedule (PANAS; Watson, et al., 1988), which participants completed prior to being given a blank note pad. Participants were instructed to locate a comfortable place where they would not be disturbed (e.g., their room), and were asked to write about their assigned topic area for the next 20 minutes (i.e., until the experimenter has returned). After returning (20 min later), they were handed the PANAS, and the Essay Evaluation Measure (EEM) (described below) assessing the degree to which they revealed their emotions, etc., in the essay (Pennebaker $\&$ Beall, 1986). Essays, which were marked by participant ID number, were then dropped into a cardboard box along with their responses to the PANAS and EEM. Finally, participants were instructed to meet with the first experimenter at a prearranged time on the following day for the next writing session. They then received their $\$ 5$ gift card for participating in the writing session.

Writing Sessions Two and Three. All participants were greeted for each session by the first experimenter, who reiterated the same writing instructions as in session one. As in session one, the second experimenter instructed participants to complete the PANAS before writing for 20 minutes, but remained blind to study group assignment. 
The PANAS and EEM were administered after each writing session. Participants were again asked to return for the next session on the following day.

Writing Session Four. Procedures were the same as those described for sessions two and three. Upon completing the activities described above, however, participants were given an appointment card for the two week follow-up.

Measures at Writing Sessions. Participants were asked to complete an immediate version of the Positive and Negative Affect Scale (PANAS; Watson, et al., 1988), both pre- and post-writing assignment. The PANAS assesses negative affect (NA) and positive affect (PA), using 10 items for NA and 10 items for PA. Scales are shown to have high internal consistency reliabilities, ranging from .84 to .87 for NA and from .86 to .90 for PA, and are unchanged by the time point used.

The Essay Evaluation Measure (EEM; Greenberg \& Stone, 1992) was administered at each writing session and served as manipulation check after each writing session. To test the intended effects of trauma writing, the EEM assesses participants' report, along separate 7-point scales, ranging from 1 (not at all) to 7 (a great deal), the extent to which their essays were meaningful, personal, and revealing, and the extent they had actually talked to others, had wanted to talk to others, and had actively held back from talking to others about the subject(s) in the essays. Comparisons were made between the groups to determine if the experimental group identified their writing as more meaningful, personal, and revealing of their emotions than those in the control group (Lepore, 1997). 


\section{Follow-Up Assessments}

Two-Week Follow-Up. Two weeks after the first writing session, participants were asked to complete a follow-up packet, including each questionnaire that was administered prior to writing except the demographic questionnaire and the SCID, but with a follow-up questionnaire added. The assessments were administered by the experimenter who remained blind to study condition and subjects received $\$ 10$ for their time and effort. Participants were then scheduled for a one month follow up and given an appointment card.

One Month Follow-Up. As in prior studies (Sloan \& Marx, 2004; Brown \& Heimberg, 2001), one month after writing session four, participants were asked to complete a follow-up packet, including each questionnaire that was administered prior to writing except the demographic questionnaire and the SCID. The assessments were administered by the experimenter who remained blind to study condition and subjects received $\$ 15$ for their time and effort.

Measures at Follow-Ups. As previously stated, all measures from the baseline assessment, were included, with the exception of the demographic questionnaire and the SCID. However, during follow-ups, participants completed a PANAS (which was not completed during baseline assessments), as well as a Follow-Up Questionnaire.

Follow-up Questionnaire. A follow-up questionnaire was administered at the end of the two week and one month assessment batteries. The questionnaire included both close-ended and open-ended questions addressing whether the participants found the writing helpful, whether she talked about the subject matter from her essays with anyone 
during her treatment at Rubicon, whether she continued using the same writing instructions after the study was complete, etc. Items also included questions about whether or not the participant heard about the writing instructions from other clients at Rubicon, and whether she told other clients at Rubicon about the writing instructions she had been given.

\section{Dependent Measures}

Dependent measures included change scores on the following measures: 1) trauma symptom severity, distress, depressive symptom severity, bodily symptoms frequency, and drug craving (baseline; two-week follow-up; one-month follow-up); and 2) mood rating forms (before and after each writing session and follow-up).

More specifically, trauma symptom severity was measured using the total sum scores of items assessing re-experiencing, arousal, and avoidance symptoms from the PDS. These three categories include some of the criteria used when diagnosing a current DSM-IV PTSD diagnosis. Distress levels were measured using raw scores from the BSI for each of the nine indices, as well as the Global Servity Index. Depressive symptom severity included the total sum score from the CES-D, while clinically elevated depression scores were assessed using a CES-D cut-off score of 16. Bodily symptoms and sensations (i.e., physical symptoms and health problems) were assessed using the total sum score from items on the PILL. Drug craving was measured using the sum of intensity, frequency, and craving scores in the BSCS. Finally, mood ratings (i.e., distress or negative affect) were calculated using the sum of Negative Affect items from the PANAS. 


\section{Compensation}

Experimental and control writing group participants had the opportunity to earn equal amounts of compensation for their time and efforts. All study participants received a \$5 gift card for completing the baseline assessment and a \$5 gift card for completing each of the four writing sessions (\$20 total). In addition, they received a $\$ 10$ gift card for completing the two-week follow-up session and \$15 for completing the one-month follow-up assessment. Thus, a woman who participated in all elements of the intervention and follow-ups would earn the equivalent of a \$50 gift card to a local merchant for her time and effort. Gift cards (e.g., Dollar General) were selected because cash payment is not allowed by this study site and they have been described as "reinforcing" by women who participated in previous studies at Rubicon.

\section{Critical Methodological Issues}

Several methodological issues needed to be addressed during the design of this project. They are listed below, along with a summary of the factors that contributed to the decisions made.

Minimization of cross-contamination: While cross-contamination is an inherent limitation in studies that utilize a residential treatment setting, the proposed study identified several ways to minimize and measure the impact of possible diffusion of treatment. First, participants were asked to keep confidential all written materials, and not to share the instructions given (See Appendix). In addition, the essay evaluation 
measure (EEM) served as a fidelity measure after each writing session. Also, written essay content was reviewed to determine whether instructions were followed in essays. Finally, at the two week and one month follow-ups, participants were asked whether they shared or received information about writing instructions with other Rubicon participants.

Sample representativeness: The proposed study evaluated the expressive writing paradigm with female SUD patients in a residential treatment facility. While residential treatment is no longer the normative modality of care in the US, it was determined that residential care was the most appropriate setting. Safety and methodology factors contributed to the decision. Specifically, this is the first study to examine expressive writing as a therapeutic adjunct in a female SUD population. The benefits of utilizing a residential program for participant safety and collection of follow-up data far outweighed potential limits placed on generalizability to more traditional outpatient settings. At Rubicon, residential program staff were available 24-hours a day and could intervene immediately should any adverse events (e.g., psychiatric distress) occur during the intervention or follow up periods.

Female SUD patients are an appropriate target population for a traumatic disclosure intervention, as the majority has experienced at least one event of an intensity level sufficient to potentially elicit PTSD, and SUD patients who experienced PTSD symptomatology report greater rates of medical problems, psychological symptoms, addiction severity, and PTSD-related symptomatology than non-traumatized patients (e.g., Najavits, et al., 1997). A diagnosis of PTSD, however, is not an inclusion criterion since previous expressive writing studies show the intervention is beneficial for 
individuals with a history of trauma, regardless of whether they meet PTSD diagnostic criteria (Pennebaker, et al., 1988).

Controlling for educational, linguistic, or cultural effects: Studies suggest the disclosure paradigm is as beneficial for maximum security prisoners with $6^{\text {th }}$ grade educations as it is for senior professionals with advanced degrees (Spera, et al, 1994; Richards, et al., 2000), and yields positive outcomes regardless of ethnicity or native language among college students (Pennebaker \& Chung, in press). Thus, the expressive writing paradigm, with the traditional instructions used in most expressive writing studies, was determined to be suitable for use with the present population.

Ethical issues in elicitation of traumatic memories: While decades of research suggests that eliciting traumatic memories through writing over several brief sessions may cause an initial rise in negative emotion, trauma disclosure has long-term psychological and physical health benefits (Sloan et al., 2004a; Pennebaker et al., 1986); and, no harm to participants has been encountered as a result of expressive writing studies. The rise in negative mood immediately after trauma writing has been found to be transient, and at follow-up, participants generally report that they feel happier than they did before writing (Pennebaker, et al., 1990). Therefore, negative mood scores were evaluated prior to, and immediately after, writing sessions in order to determine that eliciting traumatic memories through writing did not produce undue harm, and to further confirm that increases in distress would be short-lived.

Minimization of study attrition at follow-up: In order to limit attrition, a number of steps were taken. First, participants were fully informed of the tasks involved and 
encouraged not to participate if they cannot reasonably write for four consecutive days. Second, only those who were approved for 60 days of residential treatment at Rubicon were eligible to participate in this study, thus increasing the chance of obtaining onemonth follow-up data. (It should be noted that time approved for residential stay is determined by the referral source that is paying for the client, not by severity of drug problem.) Third, participants received gift cards for completing each session of the study ( $\$ 5$ for baseline assessment, $\$ 5$ for each of the four writing sessions), and received increased compensation for completing the two week follow-up assessment ( $\$ 10$ gift card) and the one month follow-up assessment (\$15 gift card). A choice of gift cards, phone cards and/or bus tickets has been well received in prior studies at this treatment site. Also, the one-month follow-up time frame was used for outcome measures to maximize retention rates.

It should be noted, however, that the one component of study methods was revised after the first $n=22$ participants had completed the study. Specifically, investigators determined that the residential program, which provided the context for the randomized clinical trial, provided a host of standard services. Therefore, it was important to look at the immediate impact of expressive writing on psychosocial functioning. Also, some participants were leaving treatment prematurely (against medical advice, AMA). Thus, IRB approval was obtained to add a 2-week follow-up assessment to the study protocol, allowing the investigators to collect short-term outcome data from a larger pool of study participants. 
Expectancy effects: In order to reduce unintentional expectancy effects and investigator bias, one experimenter administered baseline assessments and all writing instructions, while a second experimenter, who remained blind to the assigned writing conditions, administered mood rating measures, EEMs, and follow-up assessments.

Design of the control condition: The control group writing topics were selected based on a thorough review of previous expressive writing studies conducted with similar populations. While a neutral topic with this population is difficult, studies conducted with prison inmates, for example, found that having participants write about how they use their time served as a successful control writing topic (Richards, et al., 2000). Another topic is to have participants write in detail about their eating habits (e.g., writing exactly what they have eaten in the past week, etc.) (J. W. Pennebaker, Personal Communication, January 08,2007$)$.

Stratifying issues: In the original pilot study funded by VCU's Institute for Women's Health, stratification was not warranted with the small sample size. While ideally, stratified randomization could have been done with a larger sample size (i.e., with the addition of the R36 NIDA-funded Dissertation grant), since the study was already ongoing, procedures for randomization were left unchanged. Stratified randomization prohibits imbalance between treatment groups for identified factors that influence prognosis or treatment responsiveness (Kernan, Viscoli, Makuch, Brass, \& Horwitz, 1999). A decision was made to continue the randomization procedures as they were, and to check on baseline differences and control for them in subsequent analyses. 


\section{Data Analysis Plan}

Descriptive statistics were computed to examine subject characteristics. T-test and chi-square analyses were run to examine sample representativeness and potential generalizability. These analyses also examined whether there were significant differences between treatment conditions at baseline. T-test and chi-squares further examined whether there were group differences on demographic variables. Any identified differences were statistically controlled for in subsequent analyses. Descriptive Comparisons. T-tests and chi-squares examined rates of trauma, physical health problems, distress, depression, and drug preference in a sample of drug dependent women.

Hypothesis One. The first hypothesis predicted that women with co-morbid PTSD and/or significant trauma would have more severe psychological and physical health problems than those without co-morbid trauma. To test this hypothesis, t-tests for continuous variables and chi-square analyses for categorical variables measured whether trauma symptom severity and/or PTSD was associated with more significant physical health and psychological problems (i.e., levels of distress, depression, physical symptoms and sensations, current Substance Use Disorder) upon admittance to residential SUD treatment.

Hypothesis Two. The second hypothesis predicted that relative to control writing participants, individuals assigned to the expressive writing condition would show greater improvements in psychological and physical health at follow-up. While it was expected that there would be improvements across the board for both writing conditions as a 
function of being in residential treatment, it was further hypothesized that there would be an interaction, such that trauma writing participants would show greater reductions in post-traumatic symptom severity, health symptoms, and depressive symptoms than participants who wrote about a control topic. To evaluate whether there were differences between the expressive writing group and control writing group over time, the interactions between group and time for each dependent variable was examined. Significant interactions were followed up with a test of simple main effects to determine the nature of the interaction.

Short-term effects (measured at the two week follow-up) were tested by examining between-group differences over time using a 2 Group (writing topic: experimental; control) by 2 Time (baseline; two week follow-up) repeated-measures analysis of variance (ANOVA) separately for each outcome measure (PDS, BSI, CES-D, and PILL). Longer-term effects (measured at one month follow-up) were examined using a 2 Group (writing topic: experimental; control) by 2 Time (baseline; one month follow-up) repeated-measures analysis of variance (ANOVA) in order to examine differences over time between groups at one month follow-up on the PDS, BSI, CES-D, and PILL. Interactions effects and post-hoc analyses were examined. Follow-up contrast tests were conducted to examine between-group differences. If a dependent variable was found to be significantly different between groups at baseline, those baseline scores were controlled for using a univariate analysis of covariance (ANCOVA).

Intent-to-treat analyses were also conducted to include all participants who enrolled in the study. These analyses used the entire sample of $n=149$ randomized 
participants (82 expressive writing participants and 67 control writing participants), replacing missing outcome data for the 'non-completers' by carrying forward each participant's previous score (e.g. baseline value or two-week value).

Hypothesis Three. The third hypothesis stated that women randomized to the expressive writing group would show higher levels of immediate distress (measured immediately after each writing session) and lower levels of longer-term distress (measured at two weeks and one month post-baseline assessment) relative to those in the control group. Furthermore, while those in the expressive writing group were hypothesized to show higher levels of immediate distress, it was predicted that these increases would be shortlived, revealing no difference in pre-writing scores the following day. It was expected that groups would not differ in negative affect before writing, but that immediately after writing, the expressive writing group would show more negative affect than the control writing group; however, at follow-up this increase in negative affect was expected to decrease, and the expressive writing group would show less negative affect than the control group (Schwartz \& Drotar, 2004).

A 2 Group (Writing Topic: Expressive Writing; Control Writing) by 2 Time (preand post-writing session) by 4 Session (Writing 1; Writing 2; Writing 3; Writing 4) repeated measures multivariate analysis of variance (MANOVA) examined the interaction between pre-post writing and condition at each of the four sessions.

A repeated measures 2 Condition (writing topic: expressive writing; control writing) by 3 Time (Pre-writing; two week post-writing; one month post-writing) ANOVA was conducted in order to examine whether expressive writing participants 
experienced greater improvements in short-term and longer-term distress levels relative to those in the control group.

Fidelity Monitoring. T-tests compared the expressive writing group with the control writing group on items of the EEM to evaluate participants' self reports of their response to the experimental and control manipulation. Because the participants completed the EEM four times (after each 20 minute writing period), the mean of the four days for each participant was used in analyses. The experimental group was expected to report their essays as more meaningful, personal, revealing, and more likely to be discussed with others, that they have the more desire to discuss the topics of their essays with others, and that they more actively held back from talking with others (Schwartz \& Drotar, 2004). 


\section{Results}

\section{Demographics}

Table 1 summarizes demographic characteristics for the entire sample and separately for participants randomized to the expressive writing and control writing conditions. Overall, women were in their mid-30's (mean 36.3 years, SD = 8.6); had less than a high school education (mean 11.2 years, $\mathrm{SD}=1.8$ ); were single/never married (92.7\%); and over 2/3rds (70\%) identified their race as African-American. Comparisons for women randomized to the expressive writing and control writing conditions were made using independent t-tests for continuous measures and chi-square analyses for categorical measures. Only one group difference was found, with expressive writing group women being approximately 3 years older than control group women, $t(148)=$ $2.3, p<.05$. 
Table 1

Participant Characteristics: Expressive Writing $(n=82)$ and Control Writing $(n=67)$

\begin{tabular}{|c|c|c|c|}
\hline & $\begin{array}{c}\text { Expressive } \\
\text { Writing } \\
\% \text { or } \underline{M}(S D)\end{array}$ & $\begin{array}{c}\text { Control } \\
\text { Writing } \\
\% \text { or } \underline{M}(S D)\end{array}$ & $\begin{array}{l}\text { Total Sample } \\
\% \text { or } \underline{M}(\mathrm{SD})\end{array}$ \\
\hline Age (years) & $37.7(8.7)$ & $34.6(8.2)^{*}$ & $36.6(8.3)$ \\
\hline Education (last grade completed) & $11.5(1.6)$ & $10.9(2.0)$ & $11.2(1.8)$ \\
\hline \multicolumn{4}{|l|}{ Marital Status } \\
\hline Married & $4.4 \%$ & $9.8 \%$ & $7.3 \%$ \\
\hline Single/Never Married & $95.6 \%$ & $90.2 \%$ & $92.7 \%$ \\
\hline \multicolumn{4}{|l|}{ Race } \\
\hline$\%$ Caucasian & $25.6 \%$ & $23.5 \%$ & $24.7 \%$ \\
\hline$\%$ African-American & $69.5 \%$ & $70.6 \%$ & $70.0 \%$ \\
\hline$\%$ Hispanic & $1.2 \%$ & $0.0 \%$ & $0.7 \%$ \\
\hline$\%$ Other & $3.7 \%$ & $5.9 \%$ & $4.7 \%$ \\
\hline
\end{tabular}

\section{Data Analysis}

Outliers and Tests of Normality. Frequency distributions and univariate statistics were examined for evidence of non-normality and outliers. Using centered leverages, externally studentized residuals, DFFITS, DFBETAS, and by visually inspecting the data plotted on histograms, outliers were detected on the BSI and the negative affect scale of the PANAS measure. Specifically, over the course of the three assessments (baseline, 
two-week follow-up, one month follow-up) outliers were detected on four of the ten indices of the Brief Symptom Inventory ( $\mathrm{z}$ scores > 3.29), including: $n=1$ on the PHOB index at baseline $(\mathrm{z}=3.6), n=1$ on the PHOB index at two weeks $(\mathrm{z}=3.3)$, and $n=2$ on the PHOB index at one month $(\mathrm{z}=3.7$ and 3.4$) ; n=1$ on the HOS at two weeks $(\mathrm{z}=3.5)$ and $n=1$ on the HOS at one month $(\mathrm{z}=3.8) ; n=1$ on the SOM at baseline $(\mathrm{z}=3.6)$, and $n=1$ on the SOM at one month $(\mathrm{z}=3.8)$; and $n=2$ on the ANX at one month $(\mathrm{z}=3.7$ and 3.5). The Positive and Negative Affect Scale (PANAS) also revealed outliers at various timepoints, including: $n=1$ at one month $(\mathrm{z}=3.6) ; n=3$ at two week follow-up $(\mathrm{z}=5.3,3.8,3.6) ; n=1$ at writing session four, time one $(\mathrm{z}=3.6) ; n=1$ at writing session three, time two $(\mathrm{z}=3.35) ; n=2$ at writing session three, time one $(\mathrm{z}=3.86$, $3.59) ; n=3$ at writing session two, time one $(\mathrm{z}=3.85,3.7,3.59) ; n=2$ at writing session one, time one $(\mathrm{z}=3.64,3.38)$. Outliers found for any Brief Symptom Inventory raw scores or any of the negative affect items from the PANAS were changed to represent 3 standard deviations above the mean (Cohen, Cohen, West, \& Aiken, 2003).

Kolmogorov-Smirnov and Shapiro-Wilk statistics were used to test for normality of the data. Skewness and kurtosis were also examined by dividing kurtosis and skewness by their standard errors. These tests confirmed that study data were normally distributed.

\section{Success of Randomization.}

While $n=150$ women provided informed consent, one participant was never randomized to a writing condition because she became ill after completing the baseline assessment. Out of $n=149$ participants who completed randomization, $n=82(55 \%)$ 
were randomized to the expressive writing condition and $n=67(45 \%)$ were randomized to the control writing condition.

Analyses were run to examine whether there were significant baseline differences between women randomized to the experimental and control groups. No significant differences were found in number of reported traumatic events, trauma symptom severity, or a PTSD diagnosis $(p>.05)$. Also, no significant differences were found for frequency of common physical symptoms and sensations (physical health problems) or type(s) of substance use disorders (all $p>.05$ ). Furthermore, the two groups did not differ in intensity, frequency, or length of craving (as measured by the BSCS) for their most significant current substance use problem(s) over the past 24 hours (all $p>.05$ ).

Comparison of BSI baseline scores for women randomized to either the expressive writing or control writing group found only one significant group difference across all nine BSI indices. Specifically, women randomized to the expressive writing condition had significantly higher Anxiety dimension scores at baseline compared with those in the control writing condition $(M=1.3$ [SD $=1.1]$ versus $M=0.8$ [SD $=0.8]$, respectively), $t(148)=3.0, p<.005$.

Similarly, women in the expressive writing condition obtained higher CES-D depression scores than those randomized to the control writing condition $(M=25.2[\mathrm{SD}=$ $12.0]$ versus $M=21.1[\mathrm{SD}=10.9]$, respectively), $t(148)=2.2, p<.05$. A chi-square revealed that there were no significant differences in the number of participants with a clinically elevated depression level (i.e. $\geq 16$ cutoff on the CESD) between conditions $(76.8 \%$ versus $67.2 \%), p>.05$. 


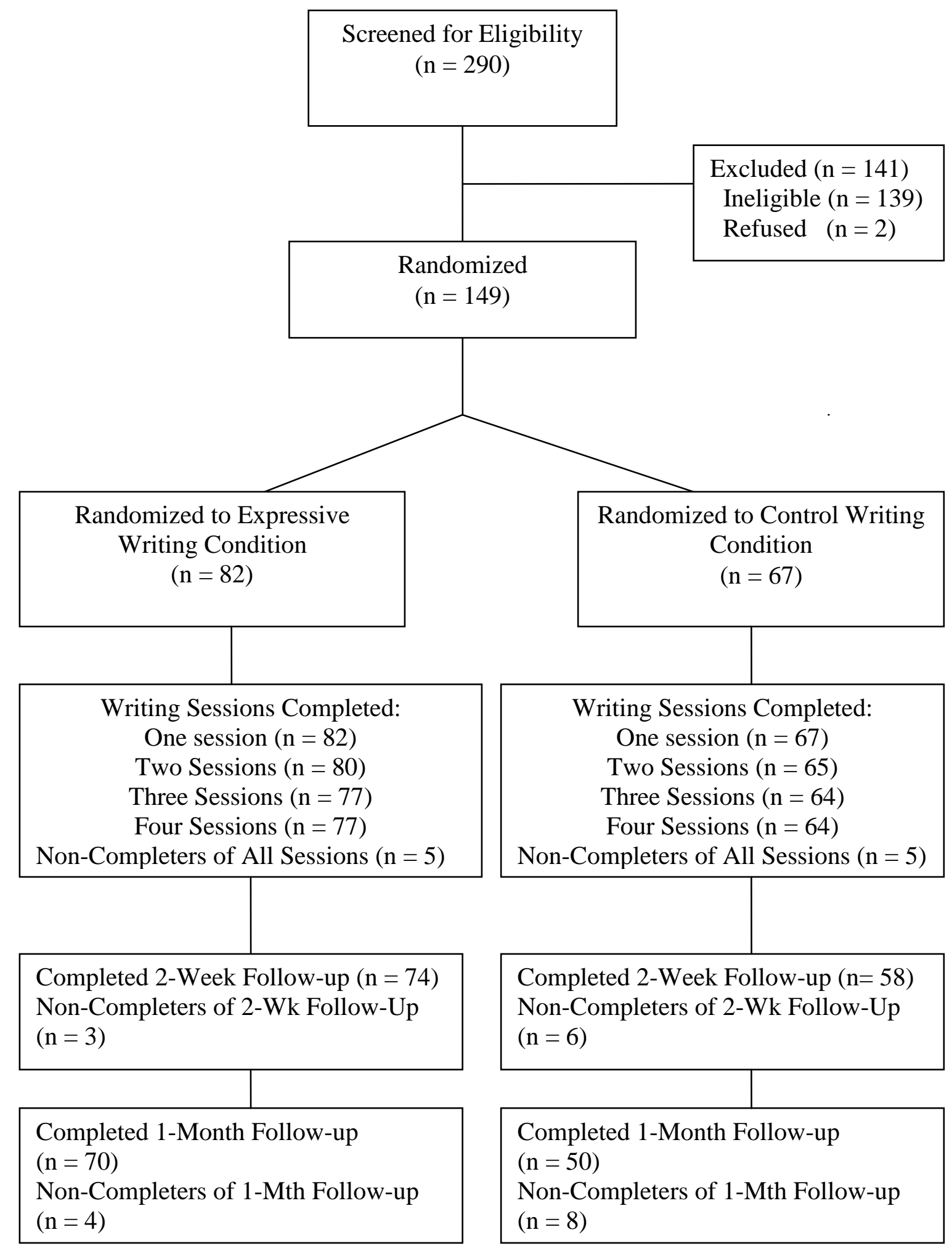

Figure 1. Flow chart of participants through study 


\section{Attrition Differential}

Writing Session Attrition. As depicted in Figure 1, rates of compliance with the intervention and control conditions were quite high, with $(n=149)$ participants completing at least one writing session and almost all of the participants $(n=145 ; 97.3 \%)$ completing more than one writing session. Specifically, $97.6 \%$ of expressive writing participants $(n=80)$ and $97.0 \%$ of control writing participants $(n=65)$ completed at least two writing sessions. There were $n=4(n=2$ [2.4\%] expressive writing and $n=2$ [3.0\%] control writing) participants who did not complete more than one writing session. Of these participants, $n=1$ expressive writing participant left Rubicon against medical advice (AMA), and the remaining $n=3$ participants did not complete more than one writing session due to medical reasons (e.g., left the facility for the day to go to the doctor, came down with the flu, etc.), which precluded these participants from completing four writing sessions over consecutive days.

A majority of the participants $(n=141 ; 94.6 \%)$ completed more than two writing sessions ( $n=77$ expressive writing participants [93.9\%] and $n=64$ control writing participants [95.5\%]). There were $n=4(n=3$ expressive writing [2.5\%] and $n=1$ control writing [1.5\%]) participants who did not complete more than two writing sessions. Reasons for not completing more than two writing sessions were similar to reasons stated above ( $n=1$ expressive writing participant left Rubicon AMA, while the remaining $n=3$ participants did not complete more than two writing sessions due to medical reasons precluding them from writing over four consecutive days). 
The remaining $n=141(94.6 \%)$ participants completed all four writing sessions. T-tests and chi square analyses revealed no significant differences between those who completed all writing sessions versus those who did not complete all writing sessions, $p>$ .05. Thus, there appear to be no systematic differences in completer versus noncompleter rates for the four consecutive writing sessions. In addition, participants who did not complete the study did so for reasons outside of the study (e.g. left the facility AMA or became sick and/or had to go to the doctor/hospital during the first week of residential treatment, and therefore could not complete all writing sessions on four consecutive days). A priori we had made the decision to continue to follow these women to identify factors that contributed to participant discontinuation in the study.

Two-Week Follow-Up Attrition. A total of $n=132(n=74$ expressive writing [90.2\%] and $n=58$ control writing [86.6\%]) participants were categorized as 'completers' for all four writing sessions as well as the two-week follow-up assessment. Since a two week follow-up assessment was added into the study procedure after the first $n=22$ participants were already enrolled, a mean substitution by condition analysis was calculated for those who completed a one month follow-up assessment $(n=16)$. Since the remaining $n=8$ participants (out of the first $n=22$ ) did not complete the one month follow-up assessments, they were not included in completer analyses. There were $n=9$ participants that completed all four writing sessions, but did not complete the two-week follow-up. Reasons for not completing the two week follow-up included: leaving Rubicon AMA ( $n=7 ;[n=5$ control writing participants, $n=2$ expressive writing 
participants), due to medical reasons precluding them from being present at Rubicon ( $n=$ $1 ; n=1$ control writing participant), or due to being discharged earlier than the expected 60 days, for reasons like a death in the family or a change in admittance length $(n=1 ; n=$ 1 expressive writing participant).

Attrition for the two-week follow-up was examined for all participants on all dependent variables. T-tests and chi-square showed no significant differences between 'completers' versus 'non-completers' on any of the dependent variables, $p>.05$. When examining attrition at two-week follow-up for the expressive writing participants only, ttests and chi-square analyses revealed no significant differences between 'completers' versus 'non-completers' on any of the dependent variables, $p>.05$.

However, when examining two-week follow-up attrition for the control writing participants only, an independent samples t-test revealed that women who did not complete the two-week follow-up were significantly more depressed at baseline compared with women who completed the two-week follow-up $(M=28.0[\mathrm{SD}=6.8]$ versus $M=20.0[\mathrm{SD}=11.1]$, respectively), $t(65)=2.1, p<.05$. In addition, a 2 x 2 chisquare analysis indicated that, among women randomized to the control group, those that did not complete the two-week follow-up were significantly more likely to have had clinically elevated depression levels (i.e. $\geq 16$ cutoff on the CESD) at baseline compared with those who completed the two-week follow-up (100\% versus 62.1\%, respectively), $\chi^{2}(2, N=67)=5.1, p<.05$. There were no other significant differences between completers versus non-completers at the two-week follow-up among the control writing participants on any other dependent variables, $p>.05$. 
One Month Follow-up Attrition. A total of $n=120$ (80.5\%) $(n=70$ [85.4\%] expressive writing and $n=50$ [74.6\%] control writing) participants completed all phases of study procedures, including writing sessions as well as the two-week and one-month follow-up assessments. Reasons for not completing the one month follow-up included: leaving Rubicon AMA ( $n=6[n=4$ control writing participants, $n=2$ expressive writing participants), due to incarceration ( $n=2 ; n=2$ control writing participants), or due to being discharged earlier than the expected 60 days, for reasons like a death in the family or a change in admittance length $(n=4[n=2$ expressive writing participants, $n=2$ control writing participants]).

Attrition for the one month follow-up assessment was examined for all participants on all dependent variables. There were no significant differences between 'completers' versus 'non-completers' at one month follow-up on any of the dependent variables, $p>.05$. An examination of attrition at one-month follow-up for the expressive writing participants only, revealed no significant differences between 'completers' and 'non-completers' on any of the dependent variables, $p>.05$. An examination of attrition at one-month follow-up for the control writing participants only, also revealed no significant differences between 'completers' versus 'non-completers' on any dependent variables, $p>.05$.

\section{Descriptive Analyses}

Trauma and PTSD among Residential SUD Women. As shown in Table 2, the majority of study participants $(96 \%)$ reported at least one lifetime trauma at baseline, 
with a mean of $3.7(\mathrm{SD}=2.3)$ different types of trauma (e.g., sexual assault, non-sexual assault, serious accident, etc.). Over half (54.4\%) of the women met diagnostic criteria for current (past month) PTSD. In addition, SUD women with co-morbid PTSD reported significantly more types of trauma than SUD women without PTSD ( $M=4.6$ [2.3] versus $M=2.8[1.8]$, respectively), $t(147)=5.1, p<.005$. Furthermore, $92.6 \%$ of women with co-morbid PTSD reported two or more types of trauma versus $73.5 \%$ of those without PTSD reporting more than two different types of traumas, $\chi^{2}(2, N=149)=9.9, p<.005$. Table 2

Trauma and PTSD: Expressive Writing $(n=82)$ and Control Writing $(n=67)$

\begin{tabular}{|c|c|c|c|}
\hline & $\begin{array}{c}\text { Expressive Writing } \\
\%\end{array}$ & $\begin{array}{c}\text { Control Writing } \\
\%\end{array}$ & $\begin{array}{c}\text { Total Sample } \\
\%\end{array}$ \\
\hline At least One Trauma & $96.3 \%(n=79)$ & $95.5 \%(n=64)$ & $96.0 \%(n=143)$ \\
\hline $\begin{array}{l}\text { Mean Number of Different } \\
\text { Types of Trauma Events }\end{array}$ & $3.8(\mathrm{SD}=2.2)$ & $3.6(\mathrm{SD}=2.4)$ & $3.7(\mathrm{SD}=2.3)$ \\
\hline $\begin{array}{l}\text { PTSD diagnosis } \\
(n=81)\end{array}$ & $4.6(\mathrm{SD}=2.2)$ & $4.5(\mathrm{SD}=2.6)$ & $4.6(\mathrm{SD}=2.3)$ \\
\hline $\begin{array}{l}\text { No PTSD diagnosis } \\
(n=68)\end{array}$ & $2.8(\mathrm{SD}=1.8)$ & $2.8(\mathrm{SD}=1.8)$ & $2.8(\mathrm{SD}=1.8)$ \\
\hline PTSD Diagnosis & $58.5 \%(n=48)$ & $49.3 \%(n=33)$ & $54.4 \%(n=81)$ \\
\hline No PTSD Diagnosis & $41.5 \%(n=34)$ & $50.7 \%(n=34)$ & $45.6 \%(n=68)$ \\
\hline \multicolumn{4}{|l|}{ Trauma Symptom Severity } \\
\hline All Residents & $21.3(\mathrm{SD}=13.4)$ & $17.5(\mathrm{SD}=12.4)$ & $19.5(\mathrm{SD}=13.1)$ \\
\hline $\begin{array}{l}\text { PTSD Diagnosis Only } \\
(n=81)\end{array}$ & $29.2(\mathrm{SD}=10.3)$ & $26.4(\mathrm{SD}=9.0)$ & $28.1(\mathrm{SD}=9.8)$ \\
\hline
\end{tabular}


When lifetime trauma exposure was characterized for the sample, the most prevalent traumas included: Imprisonment (55.7\%); Sexual contact when younger than 18 with someone 5 or more years older (45.0\%); Sexual assault by a stranger $(41.6 \%)$; Sexual assault by a family member or someone the participant knew (40.3\%); Non-sexual assault by someone the participant knew (39.6\%); Non-sexual assault by a stranger (34.2\%); Serious accident or fire (34.9\%); Other traumatic event (28.2\%); Natural disaster (24.2\%); Life threatening illness (20.1\%); Torture (10.1\%); and Military combat or a war zone $(7.0 \%)$. 
Table 3

Different Types of Trauma Events: Expressive Writing $(n=82)$ and Control Writing $(n=67)$

\begin{tabular}{|c|c|c|c|}
\hline & $\begin{array}{c}\text { Expressive Writing } \\
\%\end{array}$ & $\begin{array}{c}\text { Control Writing } \\
\qquad \%\end{array}$ & $\begin{array}{c}\text { Total Sample } \\
\%\end{array}$ \\
\hline Serious Accident or Fire & $34.1 \%(n=28)$ & $35.8 \%(n=24)$ & $34.9 \%(n=52)$ \\
\hline Natural Disaster & $26.8 \%(n=22)$ & $20.9 \%(n=14)$ & $24.2 \%(n=36)$ \\
\hline $\begin{array}{l}\text { Non-sexual Assault by } \\
\text { Someone You Know }\end{array}$ & $34.1 \%(n=29)$ & $35.8 \%(n=30)$ & $39.6 \%(n=59)$ \\
\hline $\begin{array}{l}\text { Non-sexual Assault by a } \\
\text { Stranger }\end{array}$ & $39.0 \%(n=32)$ & $28.4 \%(n=19)$ & $34.2 \%(n=51)$ \\
\hline $\begin{array}{l}\text { Sexual Assault by a Family } \\
\text { Member or Someone You } \\
\text { Know }\end{array}$ & $36.6 \%(n=30)$ & $44.8 \%(n=30)$ & $40.3 \%(n=60)$ \\
\hline Sexual Assault by a Stranger & $46.3 \%(n=38)$ & $35.8 \%(n=24)$ & $41.6 \%(n=62)$ \\
\hline Military Combat /War Zone & $1.2 \%(n=1)$ & $0.0 \%(n=0)$ & $7.0 \%(n=1)$ \\
\hline $\begin{array}{l}\text { Sexual Contact when Under } \\
18 \text { years old by someone }>5 \\
\text { years older than You }\end{array}$ & $43.3 \%(n=38)$ & $36.3 \%(n=29)$ & $45.0 \%(n=67)$ \\
\hline Imprisonment & $54.9 \%(n=45)$ & $56.7 \%(n=38)$ & $55.7 \%(n=83)$ \\
\hline Torture & $8.5 \%(n=7)$ & $11.9 \%(n=8)$ & $10.1 \%(n=15)$ \\
\hline Life Threatening Illness & $24.4 \%(n=20)$ & $14.9 \%(n=10)$ & $20.1 \%(n=30)$ \\
\hline Other Traumatic Event & $30.5 \%(n=25)$ & $25.4 \%(n=17)$ & $28.2 \%(n=42)$ \\
\hline
\end{tabular}

Various Indices of Distress among Residential SUD Women. Mean scores for the nine primary symptom dimensions of the BSI are summarized for expressive and control writing conditions, as well as the total sample in Table 4 . Scores fell around the $50^{\text {th }}$ percentile range ( $\mathrm{T}$ scores around 50) compared with BSI's inpatient psychiatric female 
norms (Derogatis, 1979), and, with the exception of the BSI Anxiety index, there were no other significant differences between the expressive writing and control groups at baseline.

Table 4

Brief Symptom Inventory: Expressive Writing $(n=82)$ and Control Writing $(n=67)$

\begin{tabular}{lccc}
\hline \multicolumn{1}{c}{$\begin{array}{c}\text { Symptom } \\
\text { Dimension/Construct }\end{array}$} & $\begin{array}{c}\text { Expressive Writing } \\
\text { Raw Score }\end{array}$ & $\begin{array}{c}\text { Control Writing } \\
\text { Raw Score }\end{array}$ & $\begin{array}{c}\text { Total Sample } \\
\text { Raw Score }\end{array}$ \\
\hline Somatization & $1.0(\mathrm{SD}=0.8)$ & $0.8(\mathrm{SD}=0.8)$ & $0.9(\mathrm{SD}=0.8)$ \\
Obsessive-Compulsive & $1.6(\mathrm{SD}=1.1)$ & $1.4(\mathrm{SD}=0.9)$ & $1.5(\mathrm{SD}=1.0)$ \\
Interpersonal Sensitivity & $1.2(\mathrm{SD}=1.0)$ & $1.0(\mathrm{SD}=1.0)$ & $1.1(\mathrm{SD}=1.0)$ \\
Depression & $1.4(\mathrm{SD}=1.0)$ & $1.1(\mathrm{SD}=1.0)$ & $1.2(\mathrm{SD}=1.0)$ \\
Anxiety & $1.3(\mathrm{SD}=1.1)$ & $0.8(\mathrm{SD}=0.8)$ & $1.0(\mathrm{SD}=1.0)^{*}$ \\
Hostility & $0.8(\mathrm{SD}=0.8)$ & $0.8(\mathrm{SD}=0.7)$ & $0.8(\mathrm{SD}=0.8)$ \\
Phobic Anxiety & $0.8(\mathrm{SD}=0.9)$ & $0.7(\mathrm{SD}=0.8)$ & $0.8(\mathrm{SD}=0.8)$ \\
Paranoid Ideation & $1.4(\mathrm{SD}=0.9)$ & $1.2(\mathrm{SD}=0.9)$ & $1.3(\mathrm{SD}=0.9)$ \\
Psychoticism & $1.3(\mathrm{SD}=1.0)$ & $1.0(\mathrm{SD}=0.8)$ & $1.2(\mathrm{SD}=0.9)$ \\
General Severity Index & $1.2(\mathrm{SD}=0.8)$ & $1.0(\mathrm{SD}=0.7)$ & $1.1(\mathrm{SD}=0.7)$ \\
\hline * $<$. 05 & & & \\
\hline
\end{tabular}

Levels of Depression and Common Physical Symptoms among Residential SUD

Women. Almost three fourths of all participants (72.5\%) obtained clinically elevated CES-D scores at baseline. Women with clinically elevated depression had significantly greater physical health symptoms (as measured by the PILL) compared to women without clinically elevated depression $(M=122.2[\mathrm{SD}=35.5]$ versus $M=101.0[\mathrm{SD}=$ 
33.2], respectively), $t(147)=3.3, p<.005$. There were no differences between the two writing conditions in the percentage of elevated depression scores or in physical health symptoms at baseline. Participants in the expressive writing condition, however, had significantly higher CES-D scores at baseline compared to those in the control writing condition ( $M=25.2[\mathrm{SD}=12.0]$ versus $M=21.1[\mathrm{SD}=10.9]$, respectively), $t(147)=2.2$, $p<.05$. Baseline score differences were statistically controlled for in subsequent testing of core hypotheses.

Table 5

Center for Epidemiological Studies- Depression Scale (CES-D) and Pennebaker Inventory of Limbic Languidness (PILL)

\begin{tabular}{lccc}
\hline & Expressive Writing & Control Writing & Total Sample \\
& $N=82$ & $N=67$ & $N=149$ \\
\hline CES-D depression score & $25.2(\mathrm{SD}=12.0)^{*}$ & $21.1(\mathrm{SD}=10.9)$ & $23.3(\mathrm{SD}=11.7)$ \\
CES-D Cutoff $\geq 16$ & $76.8 \%$ & $67.2 \%$ & $72.5 \%$ \\
PILL score & $118.8(\mathrm{SD}=37.6)$ & $113.5(\mathrm{SD}=34.2)$ & $116.4(\mathrm{SD}=36.0)$ \\
& & & \\
${ }^{*} p<.05$ & & &
\end{tabular}

Primary Drugs of Dependence among Residential SUD Women. As shown in Table 6, a large proportion of participants (80\%) indicated cocaine as one of their primary drugs of dependence (as measured by the Alcohol and Substance Use Disorders Module of the SCID). Over half (57\%) met DSM-IV criteria for Substance Dependence (current) for more than one drug. 
Table 6

DSM-IV Substance Dependence diagnosis (current): Expressive Writing $(n=82)$ and Control Writing $(n=67)$

\begin{tabular}{lccc}
\hline & $\begin{array}{c}\text { Expressive Writing } \\
\text { Alcohol }\end{array}$ & Control Writing & Total Sample \\
\hline Amphetamine/Stimulant & $32.9 \%$ & $\%$ & $\%$ \\
Cannabis & $0.0 \%$ & $1.5 \%$ & $0.7 \%$ \\
Cocaine & $80.5 \%$ & $9.0 \%$ & $10.1 \%$ \\
Hallucinogen & $1.2 \%$ & $83.6 \%$ & $81.9 \%$ \\
Inhalant & $0.0 \%$ & $0.0 \%$ & $0.7 \%$ \\
Opioid & $47.6 \%$ & $0.0 \%$ & $0.0 \%$ \\
Sedative & $6.1 \%$ & $41.8 \%$ & $45.0 \%$ \\
More than One Drug & $63.4 \%$ & $4.5 \%$ & $5.4 \%$ \\
\hline
\end{tabular}

Note: The majority of participants met criteria for more than one class of drugs

Brief Substance Craving Scale. Mean Brief Substance Craving Scale scores for the two groups and total sample are shown in table 7. An examination of participants' intensity, frequency, and length of craving for their $\operatorname{drug}(\mathrm{s})$ of choice over the past 24 hours at baseline appeared to be relatively low $(M=2.9)$. Based on the low rates of craving, this measure was dropped from further analyses examining treatment outcomes. 
Table 7

Brief Substance Craving Scale: Expressive Writing $(n=82)$ and Control Writing $(n=67)$ Expressive Writing Control Writing Total Sample

\begin{tabular}{cccc}
\hline Craving Scale (1 ${ }^{\text {st }}$ Drug $)$ & $3.1(\mathrm{SD}=3.6)$ & $2.7(\mathrm{SD}=3.2)$ & $2.9(\mathrm{SD}=3.4)$ \\
Craving Scale $\left(2^{\text {nd }}\right.$ Drug $)$ & $2.4(\mathrm{SD}=3.8)$ & $2.1(\mathrm{SD}=3.3)$ & $2.3(\mathrm{SD}=3.6)$ \\
Craving Scale $\left(3^{\text {rd }}\right.$ Drug $)$ & $1.1(\mathrm{SD}=3.2)$ & $1.0(\mathrm{SD}=2.5)$ & $1.0(\mathrm{SD}=2.9)$ \\
\hline
\end{tabular}

\section{Hypothesis One}

The first hypothesis stated that trauma symptom severity and/or PTSD would be associated with more significant psychological and physical health problems in upon admittance to residential SUD treatment.

Trauma, PTSD, and Brief Symptom Inventory/Levels of Distress. As shown in Table 8 , all nine primary BSI symptom domain scores were significantly higher among those with a current PTSD diagnosis as compared with those without PTSD, $p<.005$. 
Table 8

Brief Symptom Inventory: PTSD Diagnosis $(n=81)$ and No PTSD Diagnosis $(n=69)$

\begin{tabular}{|c|c|c|c|}
\hline \multirow{3}{*}{$\begin{array}{l}\text { Symptom } \\
\text { Dimension/Construct }\end{array}$} & PTSD & No PTSD & \multirow[t]{3}{*}{$P$} \\
\hline & Raw Score & Raw Score & \\
\hline & Mean (SD) & Mean (SD) & \\
\hline Somatization & $1.2(\mathrm{SD}=0.9)$ & $0.7(\mathrm{SD}=0.7)$ & $p<.005$ \\
\hline Obsessive-Compulsive & $1.8(\mathrm{SD}=1.1)$ & $1.2(\mathrm{SD}=0.9)$ & $p<.005$ \\
\hline Interpersonal Sensitivity & $1.4(\mathrm{SD}=1.1)$ & $0.8(\mathrm{SD}=0.8)$ & $p<.005$ \\
\hline Depression & $1.5(\mathrm{SD}=1.0)$ & $0.9(\mathrm{SD}=0.9)$ & $p<.005$ \\
\hline Anxiety & $1.4(\mathrm{SD}=1.0)$ & $0.6(\mathrm{SD}=0.7)$ & $p<.005$ \\
\hline Hostility & $0.9(\mathrm{SD}=0.9)$ & $0.6(\mathrm{SD}=0.6)$ & $p<.005$ \\
\hline Phobic Anxiety & $1.0(\mathrm{SD}=0.9)$ & $0.4(\mathrm{SD}=0.6)$ & $p<.005$ \\
\hline Paranoid Ideation & $1.5(\mathrm{SD}=1.0)$ & $1.0(\mathrm{SD}=0.7)$ & $p<.005$ \\
\hline Psychoticism & $1.5(\mathrm{SD}=1.0)$ & $0.8(\mathrm{SD}=0.7)$ & $p<.005$ \\
\hline Global Severity Index & $1.4(\mathrm{SD}=0.8)$ & $0.8(\mathrm{SD}=0.6)$ & $p<.005$ \\
\hline
\end{tabular}

Trauma, PTSD, and Depression. Consistent with hypothesis one, regardless of PTSD diagnosis, women with greater trauma symptom severity (i.e., re-experiencing, avoidance, arousal symptoms) reported more severe depression as measured by CES-D scores at baseline, $r(149)=7.3, p<.005$. Analyses further revealed that women with PTSD reported significantly greater depression levels as compared to those without $\operatorname{PTSD}(M=26.4[S D=11.9]$ versus $M=19.7$ [10.3], respectively $), t(147)=3.7, p<.005$. In addition, women with clinically elevated depression levels (CES-D $\geq 16$ ) reported 
greater trauma symptom severity as compared with those without clinically elevated depression levels $(M=23.4[S D=12.6]$ versus $M=9.7$ [SD $=7.9]$, respectively $), t(147)$ $=6.5, p<.0005$.

Physical Symptoms/Health Problems and Trauma and PTSD. Consistent with predictions, women with greater trauma symptom severity (i.e., re-experiencing, avoidance, arousal symptoms), regardless of whether or not they met criteria for current PTSD, reported more severe physical health problems as measured by the PILL at baseline, $r(149)=6.1, p<.0005$. Furthermore, women with a current DSM-IV PTSD diagnosis reported more severe physical health problems as compared with those without $\operatorname{PTSD}(M=129.0[S D=35.3]$ versus $M=101.4[S D=31.1]$, respectively $), t(147)=5.0$, $p<.0005$.

Trauma, PTSD, SUD Type, and Drug Craving among Residential Drug Dependent Women. Contrary to hypotheses, no relationship was found between trauma symptom severity and type(s) of SUDs, $p>.05$. PTSD diagnosis also did not vary by type(s) of SUDs, $p>.05$. Drug craving also did not vary as a function of PTSD diagnosis, $p>.05$.

\section{Hypothesis Two}

Another goal of the present study was to determine if women who engaged in expressive writing showed greater improvements in psychological and physical health at 
follow-up. It was expected that as a whole, there would be improvements across the board as a function of being in residential treatment. However, it was hypothesized that there would be an interaction, such that trauma writing participants would show greater reductions in post-traumatic symptom severity, health symptoms, and depressive symptoms than participants who wrote about a neutral topic. The mean baseline, twoweek, and one month follow-up scores for completers are listed in Table 11.

\section{Trauma Symptom Severity.}

Short-term Effects. Consistent with hypothesis two, women in the expressive writing condition reported significantly greater reductions in levels of traumatic stress at the two week follow-up compared to women in the control writing condition, $F(1,130)$ $=5.6, p<.05$, partial eta $^{2}=.041$. Planned contrasts demonstrated that participants who were in the expressive writing condition reported significantly greater reductions in trauma symptom severity scores (from $M=21.2[\mathrm{SD}=13.7]$ to $M=17.5$ [SD = 12.4]), whereas those in the control writing condition did not (from $M=18.4$ [SD = 12.6] to $M=$ $18.9[\mathrm{SD}=11.8]), p<.05$. Intent-to-treat analyses also revealed significantly greater reductions in levels of traumatic stress among women in the expressive writing condition compared to women in the control writing condition at two week follow-up, $F(1,148)=$ $5.8, p<.05$.

Follow-up tests showed significant decreases in trauma symptom severity, form baseline to two week follow-up scores, among expressive writing participants, $F(1,73)=$ $8.7, p<.005$, partial eta ${ }^{2}=.106$. Follow-up tests showed no significant decreases from 
baseline to two week follow-up scores among control writing participants, $F(1,57)=$ $0.2, p>.05$

Longer-term Effects. There was no significant interaction effect on trauma symptom severity at the one-month follow-up, $F(1,119)=0.7, p>.05$. Consistent with completer analyses, intent-to-treat analyses revealed no significant differences in reductions of trauma symptom severity, $F(1,147)=0.6, p>.05$.

While women in the expressive writing condition did not differ from controls in reductions of trauma symptom severity at the one month follow-up, participants from both groups revealed significant improvements in trauma symptom severity scores at the one month follow-up. A repeated measures ANOVA for the experimental group only revealed a significant decrease from baseline to one month follow-up scores, $F(1,70)=$ $10.6, p<.005$, partial eta ${ }^{2}=.133$. The control group only also showed a significant decrease from baseline to one month follow-up scores, $F(1,50)=8.5, p<.006$, partial $\operatorname{eta}^{2}=.148$.

\section{Depression Levels.}

Short-term Effects. Since CES-D depression scores were significantly different between groups at baseline, a univariate analysis of covariance (ANCOVA) controlled for baseline depression scores. Contrary to hypotheses, there was no significant interaction effect on depression scores between the two writing groups at the two-week follow-up, $F(1,131)=0.9, p>.05$. Similarly, intent-to-treat analyses revealed no 
significant difference between groups at the two week follow-up, $F(1,148)=1.4, p>$ .05 .

Longer-term Effects. Consistent with short-term effects, there were no significant differences in decreases in depression scores between the two writing groups at the one month follow-up, $F(2,119)=0.2, p>.05$. Intent to treat analyses were also nonsignificant, $F(2,148)=0.01, p>.05$.

However, participants from both groups revealed significant improvements in depression scores at the one month follow-up. A repeated measures ANOVA for the experimental group only revealed significant improvements in depression from baseline to one month follow-up, $F(1,70)=16.8, p<.005$, partial eta ${ }^{2}=.195$. A repeated measures ANOVA for the control group only also revealed a significant improvement from baseline to one month follow-up scores, $F(1,50)=8.8, p<.006$, partial eta $^{2}=.152$.

\section{Physical Health Problems.}

Short-term Effects. Contrary to hypotheses, there were no significant interaction effects over time in the reduction of physical health symptoms and sensations between the two conditions, $F(1,129)=.01, p>.05$. Intent-to-treat analyses also revealed nonsignificant findings, $F(1,147)=.02, p>.05$.

Longer-term Effects. Consistent with short-term effects, there were no significant interaction effects in the reduction of physical health symptoms and sensations at the one month follow-up between conditions, $F(1,118)=1.0, p>.05$. Intent-to-treat analyses at one month follow-up revealed similar non-significant findings, $F(1,147)=.8, p>.05$. 
A repeated measures ANOVA for the experimental group only revealed significant improvements in physical health symptoms and sensations from baseline to one month follow-up, $F(1,70)=18.1, p<.005$, partial eta $^{2}=.208$. However, a repeated measures ANOVA for the control group only showed no significant improvements from baseline to one month follow-up scores, $F(1,50)=2.8, p>.05$.

Table 9

Effects of Expressive Writing versus Control Writing on Short-term and Longer-term Follow-Up

\begin{tabular}{|c|ccc|ccc|}
\hline Groups & \multicolumn{3}{|c|}{ Expressive Writing } & \multicolumn{3}{c|}{ Control Writing } \\
& $\begin{array}{c}\text { Pre- } \\
\text { Mean } \\
\text { (SD) }\end{array}$ & $\begin{array}{c}\text { 2 week- } \\
\text { posttest } \\
\text { Mean } \\
\text { (SD) }\end{array}$ & $\begin{array}{c}\text { 1 month } \\
\text { follow-up } \\
\text { Mean } \\
\text { (SD) }\end{array}$ & $\begin{array}{c}\text { Pre- } \\
\text { Mean } \\
\text { (SD) }\end{array}$ & $\begin{array}{c}\text { 2 week- } \\
\text { posttest } \\
\text { Mean } \\
\text { (SD) }\end{array}$ & $\begin{array}{c}\text { Follow-up } \\
\text { Mean (SD) }\end{array}$ \\
\hline Trauma & 21.2 & $17.5 *$ & 16.5 & 18.4 & 18.9 & 13.9 \\
Symptom & $(13.7)$ & $(12.4)$ & $(12.9)$ & $(12.6)$ & $(11.8)$ & $(12.0)$ \\
Severity & & & & & & \\
\hline Depression & 24.8 & 21.2 & 18.3 & 20.0 & 20.3 & 15.4 \\
& $(11.8)$ & $(10.1)$ & $(11.6)$ & $(11.1)$ & $(12.1)$ & $(10.2)$ \\
\hline PILL & 117.4 & 110.9 & 103.7 & 116.3 & 109.7 & 108.2 \\
& $(38.0)$ & $(35.7)$ & $(39.1)$ & $(34.1)$ & $(27.2)$ & $(35.7)$ \\
\hline
\end{tabular}

$* p<.05$

Brief Symptom Inventory. Having demonstrated that the groups were essentially similar and therefore comparable on all BSI indices, with the exception of the Anxiety BSI index, repeated measures analyses of variance (ANOVAs) examined baseline to twoweek follow-up differences between groups as well as baseline to one month follow-ups between the two groups. A univariate analysis of covariance (ANCOVA) was used to 
control for baseline BSI Anxiety Index scores. The mean baseline, two-week, and one month follow-up scores for completers are listed in Table 10.

Anxiety Index. Consistent with hypotheses, the expressive writing group reported significantly greater reductions on BSI Anxiety Index levels than the control group, $F(1$, $129)=6.5, p<.05$, partial eta ${ }^{2}=.048($ See Table 10). Planned contrasts revealed that women in the expressive writing condition showed significant improvements in BSI Anxiety scores, $t(129)=-.288, p<.05$. Intent-to-treat analyses showed similar findings, $F(1,148)=6.5, p<.05$, partial $_{\text {eta }}^{2}=.043$. While short-term anxiety improvements differences existed between groups, analyses revealed no significant differences in anxiety reduction at the one-month follow-up, $F(1,119)=0.1, p>.05$. Intent to treat analyses also revealed no interaction effects at the one month follow-up, $F(1,148)=0.1$, $p>.05$. However, analyses revealed significant improvements in BSI Anxiety Index levels from baseline to one month follow-up for both groups. Thus, overall improvements in anxiety were seen for the expressive writing group, $F(1,70)=15.3, p<$ .005 , partial eta ${ }^{2}=.181$, as well as the control writing group, $F(1,50)=6.0, p<.05$, partial eta ${ }^{2}=.109$

Somatic Index. Analyses showed a trend approaching a significant interaction effect for the Somatic Index, $F(1,130)=3.9, p=.05$. More specifically, while the control group revealed a significant increase in Somatic symptoms from baseline to two week follow-up, $F(1,57)=4.4, p<.05$, the expressive writing group revealed no significant differences in Somatic symptoms from baseline to two week follow-up, $F$ (1, $73)=0.6, p>.05$, thus revealing a possible protective factor of the writing. Intent-to- 
treat analyses revealed similar findings, $F(1,147)=3.8, p=.05$. This trend towards significance was not maintained at the one month follow-up, $F(1,118)=0.6, p>.05$. However, analyses revealed significant improvements in BSI Somatic Index levels from baseline to one month follow-up for both groups. Thus, overall improvements in somatization were seen for the expressive writing group, $F(1,70)=11.7, p<.005$, partial eta ${ }^{2}=.145$, as well as the control writing group, $F(1,50)=5.2, p<.05$, partial eta $^{2}=.095$.

Contrary to hypotheses, analyses revealed no significant interaction effects between groups over time among the remaining seven of the nine distress indices (all > $.05)$. 
Table 10

Effects of Expressive Writing versus Control Writing on BSI Index Scores: Short-term and Longer-term Follow-up

\begin{tabular}{|c|c|c|c|c|c|c|}
\hline \multirow[t]{2}{*}{ Groups } & \multicolumn{3}{|c|}{ Expressive Writing } & \multicolumn{3}{|c|}{ Control Writing } \\
\hline & $\begin{array}{l}\text { Pre- } \\
\text { Raw } \\
\text { Score } \\
\text { (SD) }\end{array}$ & $\begin{array}{l}2 \text { week- } \\
\text { Raw } \\
\text { Score } \\
\text { (SD) }\end{array}$ & $\begin{array}{c}1 \text { month } \\
\text { Raw } \\
\text { Score } \\
\text { (SD) }\end{array}$ & $\begin{array}{l}\text { Pre- } \\
\text { Raw } \\
\text { Score } \\
\text { (SD) }\end{array}$ & $\begin{array}{c}2 \text { week- } \\
\text { Raw } \\
\text { Score } \\
\text { (SD) }\end{array}$ & $\begin{array}{c}1 \text { month } \\
\text { Raw Score } \\
\text { (SD) }\end{array}$ \\
\hline Somatization & $1.0(0.8)$ & $1.0(0.8)^{*}$ & $\begin{array}{l}0.7 \\
(0.8)\end{array}$ & $0.8(0.8)$ & $1.0(1.0)$ & $0.6(0.7)$ \\
\hline $\begin{array}{l}\text { Obsessive- } \\
\text { Compulsive }\end{array}$ & $1.6(1.2)$ & $1.4(0.9)$ & $\begin{array}{l}1.4 \\
(1.1)\end{array}$ & $1.4(0.9)$ & $1.5(0.9)$ & $1.1(0.9)$ \\
\hline $\begin{array}{l}\text { Interpersonal } \\
\text { Sensitivity }\end{array}$ & $1.2(1.0)$ & $1.1(0.9)$ & $1.0(1.0)$ & $1.0(1.0)$ & $1.1(1.0)$ & $0.8(1.0)$ \\
\hline Depression & $1.4(1.0)$ & $1.2(0.8)$ & $0.9(1.0)$ & $1.0(0.9)$ & $1.0(1.0)$ & $0.6(0.7)$ \\
\hline Anxiety & $1.2(1.0)$ & $0.9(0.9)^{* *}$ & $0.9(1.0)$ & $0.8(0.8)$ & $0.9(0.8)$ & $0.6(0.6)$ \\
\hline Hostility & $0.8(0.8)$ & $0.9(0.8)$ & $0.8(0.8)$ & $0.7(0.7)$ & $0.9(0.9)$ & $0.7(0.8)$ \\
\hline Phobic Anxiety & $0.8(0.9)$ & $0.7(0.8)$ & $0.6(0.8)$ & $0.7(0.8)$ & $0.6(0.7)$ & $0.5(0.6)$ \\
\hline Paranoid Ideation & $1.4(0.9)$ & $1.4(0.9)$ & $1.3(1.0)$ & $1.2(0.9)$ & $1.3(0.9)$ & $1.0(0.8)$ \\
\hline Psychoticism & $1.3(1.0)$ & $1.2(1.0)$ & $1.0(1.0)$ & $1.1(0.8)$ & $1.0(0.9)$ & $0.7(0.8)$ \\
\hline $\begin{array}{l}\text { General Severity } \\
\text { Index }\end{array}$ & $1.2(0.8)$ & $1.0(0.7)$ & $0.9(0.6)$ & $1.0(0.7)$ & $1.0(0.8)$ & $0.7(0.8)$ \\
\hline
\end{tabular}

$* * p<.05 ; * p=.05$

\section{Hypothesis 3}

It was hypothesized that women randomized to the expressive writing group would show higher levels of immediate distress (measured immediately after each writing session) and lower levels of longer-term distress (measured at two weeks and one 
month post-baseline assessment) relative to those in the control group. Furthermore, while those in the expressive writing group were expected to show higher levels of immediate distress, it was predicted that these increases would be short-lived, revealing no difference in pre-writing scores the following day.

Consistent with hypotheses, there was a significant time by condition interaction, $F(4,144)=9.6, p<.005$, partial $\mathrm{eta}^{2}=.211$. In addition, univariate $\mathrm{F}$ tests revealed that, as predicted, the groups did not differ in negative affect prior to writing, suggesting that the immediate increase in negative affect after writing was ephemeral.

Consistent with predictions, univariate tests revealed a significant interaction between time by condition at writing session one, $F(1,147)=28.9, p<.005$, with a significant group main effect, partial eta ${ }^{2}=.16$. There was a significant interaction between time by condition at writing session two, $F(1,147)=16.1, p<.005$, with a significant group main effect, partial eta ${ }^{2}=.10$. The interaction continued to be significant at writing session three, $F(1,147)=9.9, p<.005$, with a significant group main effect, partial eta ${ }^{2}=.06$. At writing session four, however, there was no significant interaction, $F(1,147)=2.2, p>.05$. As illustrated, analyses revealed that the main effects decreased over time. By the final writing session, participants in the expressive writing condition were possibly becoming habituated, and able to write about traumatic/stressful events without having the spike in negative affect over time (e.g., emotional arousal and habituation).

However, contrary to hypotheses, results showed no significant effect of group on distress levels at either the two week or one month follow-up, $p>.05$. 
Table 11

Negative Affect: Pre-Post Writing and Follow-Up

\begin{tabular}{|c|c|c|}
\hline Session & $\begin{array}{c}\text { Expressive Writing } \\
\text { Mean (SD) }\end{array}$ & $\begin{array}{c}\text { Control Writing } \\
\text { Mean (SD) }\end{array}$ \\
\hline Writing Session 1 Time 1 & $18.7(7.9)$ & $16.2(7.2)$ \\
\hline Writing Session 1 Time 2 & $24.0(10.9)$ & $15.2(8.2)$ \\
\hline Writing Session 2 Time 1 & $17.6(8.2)$ & $13.3(4.9)$ \\
\hline Writing Session 2 Time 2 & $22.4(11.2)$ & $13.5(5.2)$ \\
\hline Writing Session 3 Time 1 & $16.8(7.7)$ & $14.1(6.2)$ \\
\hline Writing Session 3 Time 2 & $19.5(9.5)$ & $13.4(5.1)$ \\
\hline Writing Session 4 Time 1 & $17.6(8.4)$ & $13.9(6.2)$ \\
\hline Writing Session 4 Time 2 & $18.7(9.0)$ & $13.6(5.4)$ \\
\hline Two Week Follow-up & $15.2(5.1)$ & $13.8(4.8)$ \\
\hline$(n=132: N=74, N=58)$ & & \\
\hline One Month Follow-up & $15.3(7.0)$ & $13.1(4.6)$ \\
\hline$(n=120 ; n=70 ; n=50)$ & & \\
\hline
\end{tabular}

**It should be noted that the Time 1 PANAS was assessed after writing instructions were given, but prior to writing.

\section{Validity of Group Assignment}

Essay Evaluation Measure. As shown in Table 12, the expressive writing group reported their essays as more meaningful, $t(139)=9.1, p<.0005$, more personal, $t(139)$ $=13.3, p<.0005$, more revealing of one's emotions, $t(139)=18.0, p<.0005$, and more 
likely to have wanted to discuss it with others, $t(139)=8.7, p<.0005$, than did control group participants. They also reported being more likely to have actually talked with others about the event(s), $t(139)=10.0, p<.0005$, and also to have actively held back from talking with others about the events, $t(139)=4.5, p<.0005$ than did control group participants.

Table 12

Essay Evaluation Measure scores (average item answer) for Expressive Writing $(n=77)$ and Control Writing $(n=64)$

\begin{tabular}{llll}
\hline & $\begin{array}{c}\text { Expressive } \\
\text { Writing }\end{array}$ & $\begin{array}{c}\text { Control } \\
\text { Writing }\end{array}$ & \\
& Mean (SD) & Mean (SD) & \\
& $6.2(1.3)$ & $3.9(1.7)$ & $<.0005$ \\
\hline & $6.2(1.5)$ & $2.7(1.7)$ & $<.0005$ \\
\hline "The essay was meaningful." & $6.2(1.2)$ & $2.2(1.4)$ & $<.0005$ \\
$\begin{array}{l}\text { "The essay was personal." } \\
\text { "The essay was revealing of my emotions." }\end{array}$ & $4.0(1.8)$ & $1.8(1.0)$ & $<.0005$ \\
$\begin{array}{l}\text { "I wanted to talk to others about the event(s) } \\
\text { that I wrote about." }\end{array}$ & $4.1(1.9)$ & $1.5(0.9)$ & $<.0005$ \\
"I have actually talked to others about the & & & \\
$\begin{array}{l}\text { event(s) that I wrote about." } \\
\text { "I have actively held back from talking to } \\
\text { others about the event(s) that I wrote about." }\end{array}$ & $4.2(1.7)$ & $2.8(1.9)$ & $<.0005$ \\
\hline
\end{tabular}

Follow-up Questionnaire. As shown in table 13, the expressive writing group was significantly more likely than the control writing group to report finding the writing to be helpful, $\chi^{2}(2, N=120)=18.6, p<.005$, to continue journaling using the writing instructions given during the study after the writing sessions ended, $\chi^{2}(2, N=120)=$ $10.8, p<.005$, and to have talked about the subject matter from their essays with 
someone during their treatment at Rubicon, $\chi^{2}(2, N=120)=8.8, p<.005$. Most

$(85.7 \%)$ of the expressive writing participants who talked about the subject matter from their essays with someone during treatment found it to be helpful.

\section{Table 13}

Follow-up Questionnaire at One Month Follow-up: Expressive Writing $(n=70)$ and Control Writing $(n=50)$

\begin{tabular}{lcc}
\hline & $\begin{array}{c}\text { Expressive } \\
\text { Writing }\end{array}$ & Control Writing \\
& $\%$ & \\
& $98.6 \% * *$ & $72.0 \%$ \\
\hline Did you find the writing helpful? & $15.9 \% * *$ & $0.0 \%$ \\
$\begin{array}{l}\text { Did you talk about the subject matter from your essays } \\
\text { anyone during your treatment at Rubicon? }\end{array}$ & $\mathrm{n} / \mathrm{a}$ \\
$\begin{array}{l}\text { If yes, did you find it helpful to talk about the subject } \\
\text { matter from your essay? }\end{array}$ & $100.0 \% * *$ \\
$\begin{array}{l}\text { Did you continue writing using the instructions given } \\
\text { to you after the writing sessions had ended? }\end{array}$ & $42.0 \% * *$ & $14.0 \%$ \\
\hline
\end{tabular}

$* p<.005$

As a fidelity check, the Follow-Up Questionnaire asked whether participants heard any clients at Rubicon discussing the writing instructions for this study, and a total of $n=2(1.7 \%)$ reported hearing about the instructions from another client at Rubicon (both were control writing participants). The following question, which asked what the participants 'thought' the other writing instructions were about, confirmed that these were the only two participants who heard about the other writing instructions. 


\section{Discussion}

The purpose of the present study was to collect benchmark data on whether Pennebaker's expressive writing paradigm could be applied to women currently in residential SUD treatment. The study first described rates of trauma, physical health problems, distress, depression, and drug craving in a sample of drug dependent women assessed within days of admission to residential treatment. It then examined whether comorbid PTSD and/or trauma-related symptomatology was associated with more severe psychiatric and physical health problems. Next, in a clinical trial, it compared changes in

physical and psychiatric symptom severity in women randomized to either an expressive writing or neutral topic writing control group. Both conditions were provided over 4 consecutive days in the context of residential SUD treatment. It was hypothesized that women in the expressive writing group would report greater reductions in symptoms related to PTSD than women in the neutral topic control group. Finally, the study looked at patient safety and investigated whether expressive writing increased the risk for early treatment discontinuation or increased levels of trauma-related distress and other psychological/physical health problems.

\section{Epidemiology of Trauma and PTSD in Women with SUDs}

Study participants reported rates of trauma comparable to those previously reported in the literature, supporting the rationale for the clinical trial and study hypotheses (Dansky, Saladin, Brady, Kilpatrick, \& Resnick, 1995; Hien, Cohen, \& Campbell, 2005). Specifically, the present study found nearly all women (96\%) experienced at least one lifetime traumatic event severe enough to warrant a PTSD 
diagnosis. Since co-morbid trauma seems to be the norm, rather than the exception among women in treatment for SUDs, findings confirm the importance of screening for trauma in such settings. Sadly, the majority of women with SUDs are neither evaluated for PTSD nor provided treatment for trauma (Najavits, 2006). In addition, many women underreport or deny such symptoms, resulting in missed opportunities for intervention (Gutierres \& Van Puymbroeck, 2006). Similar patterns are seen in mental health settings, where trauma is the focus of treatment and co-morbid SUDs often go undetected. Such 'split systems' of care (where mental health clinicians feel unable to sufficiently assess or address SUDs, while SUD clinicians feel unable to assess or address trauma) are the norm, despite the fact that such programs are believed to be less effective than concurrent or integrated treatment (Najavits, 2006).

Compatible with previous research (e.g. Fullilove, et al., 1993; Kessler, Sonnega, Bromet, Hughes, \& Nelson, 1995; Najavits, et al., 1997), the current study found that over half (54.4\%) of participants met diagnostic criteria for current (past month) PTSD. These rates are consistent with studies showing current PTSD among women in substance treatment settings range from $30 \%$ to $59 \%$ (Najavits, et al., 1997), compared to PTSD rates in the general population which range from 1\% to 9\% (Kessler, et al., 1995).

The present study also found that, regardless of PTSD diagnostic status, participants reported a mean of 3.7 different types of trauma events at baseline. These numbers reflect similar amounts and types of trauma events as those reported in previous research with substance dependent women, with an average of 4 different types of trauma per woman (e.g. Brown, Stout, \& Mueller, 1999). In addition, the present study found 
that number of different types of traumatic events were significantly higher in SUD women with a co-morbid current diagnosis of PTSD as compared to those without PTSD ( $M=4.6$ versus $M=2.8$, respectively). It should be emphasized that these analyses compared number of different types of traumatic events experienced, and not the overall frequency with which participants experienced traumatic events. Many of the women in the present study experienced repeated traumatic events within the same type of trauma, which is not reflected in the data. Taken together, the present study confirms that repeated trauma is pervasive among female SUDs and reinforces the need for programs to concurrently address both SUD and co-morbid PTSD/trauma, because interventions focused on only one of the two disorders may be inadequate and lead to poorer outcomes.

The specific nature and prevalence of trauma found in the present study corresponds with earlier studies (e.g., Brown, Stout, \& Mueller, 1999; Coffey, et al., 1998), and most of the traumas were of an interpersonal and violent nature. Research suggests that interpersonal trauma is more likely than accidents or natural disasters to result in co-occurring symptoms and problems that go beyond PTSD-SUDs, such as depression, somatic complaints, disassociation, impulsive behavior and anger management problems (Cohen \& Hien, 2006; Roth, Newman, Pelcovitz, van der Kolk, \& Mandel, 1997). Furthermore, if an individual is using drugs to self-medicate, once outside of residential treatment (if traumas are not properly addressed during SUD treatment) she will be more likely to experience a repeat trauma, which may increase the likelihood of relapse to drug use. Thus, by addressing trauma, we can hopefully help prevent further re-victimization. 
High rates of PTSD in women with SUDs warrant more attention from the addiction treatment community. It is imperative that counselors recognize the heterogeneous ways in which PTSD can affect their patients and drug abuse treatment. The extent and duration of symptoms, as well as the degree of social and functional impairment, co-morbidity, and somatization should be carefully monitored and evaluated (Breslau, 2001). SUD treatment providers can no longer ignore this co-morbid disorder. They must improve their approach for identifying PTSD and trauma symptoms and creative ways to link women with appropriate treatment services, either on-site or through community referral. Failure to do so is likely to reinforce premature treatment drop out and poorer (Brown, Stout, \& Mueller, 1999).

Clinical Profiles and Treatment Response in SUD Women with and without PTSD

The first hypothesis was supported by the data, with poorer health and more severe clinical profiles in SUD women with PTSD or PTSD-related symptomatology, as compared to those without such co-morbidities.

Trauma and Health among Women with SUDs. The present study revealed that SUD women with co-morbid PTSD have significantly more physical health symptoms and sensations compared to SUD women without PTSD. Thus, while SUDs are associated with a wide range of physical and mental health detriments, PTSD confers an even greater risk and detriment (Ouimette, Goodwin, \& Brown, 2006). These women have disproportionate physical health burdens and are especially susceptible to risk factors related to poor health and disability. 
The poor health of these women may be a sign of persistent obstacles toward obtaining sufficient medical attention as well as complications connected with their substance abuse or psychological problems; furthermore, there are undeniably special needs that medical providers might not identify or be prepared to sufficiently manage, given the issues faced by women who have a combination of substance abuse and trauma (Larson, et al., 2005). While there is currently concern among some SUD treatment providers that integrated trauma treatment poses a danger for increasing the patient's adverse psychiatric and physical symptoms (Hien, et al., 2004), present study findings underscore a need for interventions aimed at facilitating improved health outcomes among co-morbid SUD-PTSD patients who seek residential treatment, as these women are at greater risk for physical health problems.

Clinical Profiles among Women with SUDs and Trauma. In addition to physical health problems, the present study supports previous research showing that women with co-morbid SUD and PTSD experience greater levels of psychological distress across a host of domains compared to those without trauma histories (Clark, et al., 2001; Coffey, et al., 2005; Najavits, et al., 1997;). Since women with SUDs already experience a disproportionate amount of psychological distress compared to women in the general (non-clinical) population, these findings highlight the fact that the presence of both of these syndromes confers still greater risk and severity of psychological distress.

Scores for all nine primary dimensions of the BSI as well as the General Symptom Severity Index were significantly higher in SUD women with co-morbid PTSD as compared to those without PTSD. Again, findings are congruent with the research 
literature, which shows more severe clinical profiles and interpersonal problems in dually diagnosed females as compared to females with only one of the two disorders (Brown, et al., 1999; Najavits, et al., 1999; Ouimette, Wolfe, \& Chrestman, 1996). However, it is also important to note that there is general overlap between PTSD symptoms and BSI index scores; therefore, these findings are not too surprising, yet still reflect significant difficulties experienced by this particular population. Still, research shows that a history of abuse and trauma, regardless of gender, race, or other mental disorders or criminal activity, is related to a lower likelihood of posttreatment abstinence among adults in drug abuse treatment (Boles, Joshi, Grella, \& Wellisch, 2005). Thus, there is considerable evidence that further clinical research may be required to in order to more effectively help these women and improve interventions for handling these co-morbid problems without triggering more harm.

Depression among Women with SUDs and Trauma. Since depression is a common co-morbidity in women with SUDs, it was important to look specifically at depressive symptoms in SUD women with and without PTSD. As expected, the present study found nearly three fourths of participants (72.5\%) obtained clinically elevated depression scores at baseline assessment. Women with co-morbid PTSD reported significantly higher depression levels as compared to those without PTSD. Furthermore, women with clinically elevated depression levels $(\mathrm{CES}-\mathrm{D} \geq 16)$ reported greater trauma symptom severity as compared to those without clinically elevated depression. Therefore, the current study supports previous evidence suggesting that SUD women with a history of PTSD and trauma are more likely than those without PTSD to be 
diagnosed with depression and to report higher rates of depressive symptoms (Clark, et al., 2001).

While the present study did not formally assess for DSM-IV disorders other than PTSD and substance abuse/dependence, findings suggest that it is likely that some of these women would have met criteria for a mood disorder, as well (e.g., Major Depressive Disorder, Dysthymia). Using a sample of mothers with PTSD $(n=801)$, Breslau (2001) found that $43.2 \%$ of the women had Major Depression that was secondary or co-morbid with conditions like SUDs. In another study comparing SUD patients with and without co-morbid PTSD, 79\% of SUD patients with PTSD had Major Depressive Disorder, compared to $51 \%$ of SUD patients without co-morbid PTSD (Brown, Stout, \& Mueller, 1999). Dysthymia is also significantly more prevalent among SUD patients with PTSD as compared to SUD patients without PTSD (19\% versus 6\%, respectively) (Brown, Stout, \& Mueller, 1996).

The presence of PTSD, a SUD, and/or depression appears to increase the risk of developing the other disorder, and these findings are consistent across various kinds of trauma and substances of abuse (Najavits, Weiss, \& Shaw, 1997). While high comorbidity rates indicate that PTSD and SUDs are functionally related, the mechanisms underlying the development of this relationship is not clear (Back, Brady, Sonne, Verduin, Back, et al., 2006). While alcohol and drugs may be used to self medicate to avoid trauma reminders and related distress, the lifestyle of someone with drug use problems may predispose these individuals to endure traumatic events (Johnson, Striley, \& Cottler, 2006; Reynolds, et al., 2005). Also, depression can put women at an increased 
risk for SUDs, which can place them at-risk for trauma. Another theory suggests that an independent third factor, such as genetic and/or environmental influences, may contribute to the high co-occurrence of these problems (Brown \& Wolfe, 1994; Xian, et al., 2000). While the present study did not examine the time course for development of the various disorders and differences in age of onset, it appears that any one of these problems enhances the likelihood of the other problem co-occurring.

Drug Craving and Drugs of Dependence among Women with SUDs and Trauma. Contrary to previous research showing that violent trauma and distress are correlated with severity of drug use (e.g., Clark, et al., 2001), the present study did not find drug craving to be related to trauma. While craving is just one aspect of what would be considered 'severity' of drug use, several factors may contribute to our failure to find a relationship between craving and trauma. First, many of these women came from a controlled setting prior to enrolling in Rubicon's residential program (e.g., prison, detox, etc.). Thus, the period of abstinence from drugs prior to treatment enrollment may have reduced overall drug craving scores (Da Silveira, Doering-Silveira, Niel, \& Jorge, 2006), possibly influencing how much participants were 'currently' craving the drug. In addition, some research suggests that overall low craving scores tend to be found once a patient is out of her "natural environment" (Garavan, et al., 2000). Furthermore, it is possible that the null findings are in part due to the instrument used to measure craving.

The measure that was used revealed a low reporting of drug craving and relatively low variability. Therefore, the Brief Substance Craving Scale (Somoza, 1999) that was used to measure craving was essentially dropped from further analyses in the present 
study. It is believed that there was a low ability to detect drug craving appropriately with the brief measure used.

The present study also did not find significant differences in drug(s) of dependence between women with co-morbid PTSD and women without PTSD. While some studies have found that "harder" drugs, such as cocaine and heroin, are more strongly related to trauma and PTSD (Grice, et al., 1995; Miller, Downs, \& Testa, 1993; Najavits, Weiss \& Shaw, 1997), other studies have found that PTSD and trauma severity are unrelated to drug preference and seem more generally linked to the disorder of drug dependence (Johnson, Striley, \& Cottler, 2006).

One thing to consider is that most of these women reported a primary drug of either cocaine $(81.9 \%)$ or heroin $(45.0 \%)$, and many of the women reported having more than one drug of dependence. Furthermore, while $28.9 \%$ of the women in the present study reported alcoholism and $10.1 \%$ reported marijuana dependence, many of these women used other "harder" drugs as well, contributing to rates of polydrug use/dependence. In addition, homogeneity is present in the target sample because a certain level of drug severity is generally required before residential services are offered as a treatment option. To illustrate, women generally do not get admitted into Rubicon's residential program for marijuana dependence alone. As mentioned in the Methods section, there was also a period of time when the present study excluded women with alcohol dependence as a primary problem, since alcohol dependent women were being recruited for another randomized clinical trial specific to women with an alcohol use disorder. 
Shorter Term and Longer Term Effects of Expressive Writing in SUD Women

In support of the second hypothesis, results from the present study indicate that expressive writing participants demonstrated greater reductions in post-traumatic symptom severity and anxiety scores than control writing participants. However, there were no significant interaction effects with the other psychological and physical health measures. While no group differences were found at one month follow-up, both groups showed significant positive changes, which is likely due to being in residential treatment for 30 days (Bartels \& Thomas, 1991).

Trauma Symptom Severity. In support of hypothesis two, the present study found that women in the expressive writing condition demonstrated significantly greater improvements in levels of traumatic stress compared to women in the control writing condition at the two week follow-up. More specifically, those in the expressive writing condition indicated significant reductions in trauma symptom severity levels, while control writing participants indicated no improvements in symptom severity. At the one month follow-up, however, improvements were seen regardless of writing condition, and there were no significant differences in trauma symptom severity improvements between expressive writing and control writing participants.

Overall, short-term findings were consistent with previous research with other pools of participants suggesting that writing about emotional experiences using expressive writing results in a decreased degree to which the experience is distressing or 
painful (Lepore \& Smyth, 2002; Sloan, Marx, \& Epstein, 2005). One potential reason for these findings is based on the exposure theory. This theory suggests that an individual's attempt not to think about traumatic/stressful experiences in order to avoid becoming overwhelmed by emotions (avoidance behavior), may generate further feelings of distress and fear associated with the trauma (Lepore, Greenberg, Bruno, \& Smyth, 2002). Thus, expressive writing may act as a medium that allows the individual to be exposed to unpleasant stimuli that had been previously avoided. Repeat exposure over several writing sessions may allow for habituation and extinction of negative emotional associations and/or activate the fear structure and offer corrective information to the person about the stimuli, responses, and meaning of the event (Sloan \& Marx, 2004). The concept that exposure to feared stimuli can bring about appropriate management of emotional matter appears applicable to the written disclosure paradigm, as it may help alleviate distress by conquering one's propensity to avoid or suppress upsetting memories and emotions.

While it is generally thought that exposure to the same traumatic event is essential for extinction in other exposure therapies, the expressive writing task does not require that the individual write about the same event at each writing session, suggesting that stimulus-related habituation can take place regardless (Sloan \& Marx, 2004; Lepore, et al., 2002). Studies to-date comparing instructions for participants to write about the same or different topics each day have yielded mixed results.

One recent study using a population of college students with a history to trauma, found that participants who wrote about the same trauma experience at each session 
showed improvements at follow-up, while participants instructed to write about a different trauma each day did not (Sloan, Marx, \& Epstein, 2005). Other studies have obtained more robust results when participants were allowed to choose their writing topic at each session (Pennebaker, 1997; Smyth, 1998).

While the present study did not instruct women to write about the same event or different events across the four writing sessions, further investigation of the essays are needed to determine how many women focused on a single event versus multiple events. The relationship between essay topic consistency and outcome measures warrants further study, recognizing that other factors (types of traumas experienced, recency of trauma) may influence participant decision making. Also, some women may benefit most by writing about the same trauma across all sessions, while others may be better served by writing about different events at each session (Sloan, et al., 2005).

While the present study found significant short-term improvements in trauma symptom severity among expressive writing participants relative to the control writing participants, significant differences between conditions were not maintained at the longterm follow-up. These findings are consistent with the literature that suggests that benefits made through the written disclosure task may dissipate rapidly over time (Sloan \& Marx, 2004).

The present study is unique, however, in that it was conducted in the context of residential treatment, which, in and of itself, is likely to produce improvements in physical and psychosocial functioning over time. Thus, while expressive writing and control writing groups did not differ at one month follow-up, both groups demonstrated 
positive changes on core outcome measures. Also, results affirm that expressive writing did not have a detrimental effect at one month follow-up.

The importance of short-term improvements at two weeks versus one month, should not be discounted, particularly since we are dealing with a clinical population and a chronic disorder. While demonstrating significant improvements over the control writing condition at the one-month follow-up would have been ideal, it is not surprising that such severe trauma and/or PTSD (as was found in the present study), was not alleviated by such a brief psychosocial intervention.

The ability to reduce trauma symptom severity early in drug treatment can be beneficial in many ways (e.g., increasing patient ability to concentrate during counseling sessions, reducing risks for leaving treatment prematurely, reducing risks for relapse to drug use). Thus, future studies should explore whether extending the intervention in ways that maintain or further 'boost' short-term improvements at a later time, possibly through more writing sessions distributed over the course of SUD treatment, may enhance longer-term effects.

Koopman and colleagues (2005), for instance, suggested that writing for a total of 80 minutes (20 min sessions over 4 consecutive days) was possibly not enough to ameliorate PTSD symptoms in general among women with intimate partner violence (IPV), and that instructing women to write about the IPV experiences (versus writing about any traumatic/stressful event) may have been more helpful in reducing PTSD symptoms. In another study examining the utility of expressive writing with individuals with PTSD (clinical population), Smyth, Hockemeyer \& Tulloch (2008) found decreases 
in PTSD symptom severity from baseline to follow-up for both groups, but like us, found no significant difference between the expressive writing and control writing group in PTSD symptom changes at longer-term follow-up.

Depression. Contrary to hypotheses, there were no significant interaction effects over time (at two weeks or at one month) in the reduction of depressive symptoms between the two writing conditions. These findings stand in contrast to previous studies of depression in college undergraduates (Gortner, Rude, \& Pennebaker, 2006; Lepore, 1997, Sloan, Marx, \& Epstein, 2005). They are congruent, however, with previous research targeting clinical populations (Frisina, Borod, \& Lepore, 2004). For instance, a meta-analysis of expressive writing in clinical and medical populations (four clinical sample studies; five medical sample studies) found expressive writing was less effective for psychiatrically as compared to physically ill patients (Frisina, et al., 2004). In addition, it is important to remember that both the experimental and control groups in the present study had significant reductions in depressive symptoms at one month follow-up, which was likely associated with having spent 30 days drug free in a supportive residential SUD treatment setting.

As the number of expressive writing studies with clinical populations grows, findings have begun to indicate that supplemental interventions may be required in order to enhance the benefits gained from the general expressive writing task. It is also more apparent that there are potential moderating variables (e.g. instructional set for written disclosure) and mediating variables (e.g., role of cognitive processing, which can be analyzed through content analysis), that may impact the beneficial effects of the writing 
paradigm (Frisina, et al., 2004). Understanding these moderating variables may help us to better understand how expressive writing can effectively influence depression levels.

For instance, while Gortner and colleagues (2006) found improvements in depression among those in the expressive writing condition (versus the control writing condition) in a depression-vulnerable college population, these improvements were seen only among less expressive participants (those with high Emotion Regulation Questionnaire Suppression scores), and were mediated by changes is brooding but not reflection scores. Thus, results suggest that individuals who were less expressive may have had a greater need for the opportunities that expressive writing treatment offers.

Physical Health Problems. Contrary to hypotheses, there were no significant interaction effects over time (two week or one month) in the reduction of physical health symptoms and sensations between the two writing conditions. This finding conflicts with an extensive body of research suggesting positive physical health benefits from expressive writing (e.g., Pennebaker \& Beall, 1986; Smyth, et al., 1999). However, results showed overall improvements in physical symptoms and sensations for all participants, regardless of group assignment, at the one month follow-up assessment, which is likely due to having received 30 days of residential care.

While we did not find the health improvements we expected from the expressive writing task, it is also important to remember that the present study uses a sample of women who, generally speaking, were receiving little to no healthcare treatment prior to admittance at Rubicon. Once admitted into the residential treatment facility (baseline assessments were conducted within the first week of treatment), however, these women 
began receiving psychotherapy and/or medication, which likely influenced symptoms and self-reports at follow-up, above and beyond improvements we may otherwise witness as a result of the disclosure paradigm.

It is also important to note that these participants are at-risk for chronic health problems, such as diabetes, hepatitis C, HIV, etc., (unfortunately, the present study did not collect data on this information), and there was likely a wide variation of health problems (both chronic and acute) at the start of treatment among these women. As a result, the present sample could have had an elevated level of pre-existing variance (noise), which as a result, could have inhibited our capability to interpret the effectiveness of expressive writing on health (Frisina, et al., 2004).

Another thing worth mentioning is that there is a full-time nurse at Rubicon working hard to address all medical-related needs. Thus, participants received continuous care for their medical needs as well as medication to address physical illness from the moment they arrived at Rubicon. As noted, the present study found improvements in health related symptoms for people in both writing conditions. Therefore, what our findings suggest is that the writing paradigm did not have a large enough impact on physical health symptoms and sensations above the improvements generally seen.

There are also many ways that previous expressive writing studies have examined health outcomes. Self-report data is one way to examine health, but certainly not the only way. For instance, many studies have examined physician visits, physiological markers, and behavioral makers in order to assess health outcomes. Another way to have 
measured physical health with the present sample would have been to record the number of groups missed due to feeling ill (we do not have actual numbers, but it is not uncommon for residents to miss groups due to feeling ill), which may have provided a different/more effective way of measuring health improvements (Pennebaker, 1997). Furthermore, longer term follow-up outside of residential care, would be another way to assess for physical health effects.

Anxiety Index from Brief Symptom Inventory. Consistent with hypotheses, the expressive writing group reported significantly greater reductions in BSI Anxiety Index levels than the control writing group at the two week follow-up. Contrary to hypotheses, however, there were no significant main effects in anxiety at the one-month follow-up.

Short-term findings from the present study may suggest that expressive writing assists with emotion regulation through attention, habituation, and cognitive restructuring (Lepore, Greenberg, Bruno, \& Smyth, 2002). Expressive writing can potentially help influence attention toward or away from different parts of a stressor, and through repeat exposure, habituation may have occurred during writing (Lepore, et al., 2002). Foa (1997) contends that for successful habituation, an individual should undergo strong negative emotions initially, but then show steady reductions in negative emotions over time (see below in discussion of hypothesis three). Another theory suggests that cognitive restructuring may initiate changes in stress-related thoughts and appraisals. To the degree that people can cognitively incorporate stressful experiences, they should experience a decrease in and possible extinction of anxiety and intrusive thoughts. 
The one month findings on anxiety in the present study are contrary to predictions, but consistent with some research suggesting no differences between expressive writing and neutral writing conditions in anxiety reduction (Kloss \& Lisman, 2002). Studies examining essay content have also revealed that language categories, such as negative emotion words, are related with differing levels of anxiety among women in the expressive writing condition (Smith, Anderson-Hanley, Langrock, \& Compas, 2005). Based on the emotional processing theory, we might expect to find that participants who wrote about the same experience, versus different experiences across all writing sessions, would benefit most from the writing paradigm.

Somatic Index from the Brief Symptom Inventory. Analyses in the present study revealed a trend approaching significance for the BSI Somatic Index. More specifically, control writing participants revealed a significant increase in somatic symptoms at the two week follow-up, while expressive writing participants showed no changes in somatic symptoms from baseline to the two week follow-up. Thus, results might suggest that expressive writing could have a protective effect on drug dependent women during the early stages of residential treatment.

If drug dependent women are likely to reveal initial increases in somatic problems (within the first couple weeks of residential treatment), possibly as a result of their bodies readjusting to no longer being physically dependent on drugs, then written emotional disclosure may benefit women by preventing somatic deterioration. The notion that the expressive writing paradigm may serve as a protective influence is particularly noteworthy considering that there had historically been concerns over adverse reactions 
or negative experiences attributed to an integrated trauma-related and SUD treatment (e.g., Killeen, Hien, Campbell, Brown, Hansen, Jiang, et al., 2008).

Remaining BSI Indices. Contrary to hypotheses, there were no significant interaction effects over time (two week or one month) for the remaining BSI distress indices. Consistent with previous analyses, however, we did find general improvements across all indices on the BSI at the one month follow-up for both writing groups. Effects of Expressive Writing on Immediate and Longer-Term Levels of Distress

Consistent with hypothesis three, women randomized to the expressive writing condition showed greater levels of immediate distress (post-writing negative affect scores) relative to those in the control writing condition. In addition, negative affect measured prior to the writing session the next day (pre-writing negative affect scores) revealed no differences between the two writing conditions. Contrary to predictions, however, expressive writing did not produce significant longer-term improvements in negative affect over control writing. Participants in both writing conditions, however, displayed decreases in negative affect at the two-week and one-month follow-up. These longer term findings may be primarily a result of residential treatment improvements overshadowing potential group differences.

Although writing about traumatic/stressful experiences can be psychologically painful in the short run, over time there appears to be decreased negative affect associated with writing about the traumatic or stressful experience. As predicted, the groups did not differ in negative affect on days $2-4$ prior to writing, suggesting that the immediate increase in negative affect after writing was temporary. 
Increased short-term negative affect immediately following writing is consistent with previous literature in the area (Esterling, L'Abate, Murray, \& Pennebaker, 1999), and provides evidence for emotional engagement and (fear) activation (Kloss \& Lisman, 2002). Experts in the field of trauma claim that emotional engagement is essential for habituation to occur (Foa, 1997). In order for successful habituation to occur, an individual should endure strong negative emotions initially (Lepore et al., 2002), followed by gradual reductions in negative emotions within and across writing sessions, as was seen in the present study.

Such habituation may indicate desensitization to trauma-related memories and thoughts (i.e., stimulus-specific habituation), and possibly an enhanced tolerance of the individuals' negative emotional responses to stress-provoking stimuli in general (i.e., response-specific habituation; Lepore, et al., 2002). Thus, initial spikes in distress and consequent habituation may be reflective of emotional processing and enhanced selfregulation, and should be associated with improvements in one's reaction (psychological and physical) to trauma memories (Sloan, Marx, Epstein, \& Lexington, 2007).

While expressive writing did not result in significant longer term improvements in negative affect over the control writing, it is possible that instructing participants to write about the same or different experiences across all four days could have played a role in these null effects. While writing about the same experiences across all four days should enhance exposure, it has been suggested that writing about different experiences may weaken exposure (Kloss \& Lisman, 2002). Further analyses should compare distress 
ratings for those who wrote about the same traumatic/stressful experiences versus different experiences across all four days.

Since control writing participants evinced relative decreases in negative affect, results may indicate that improvements in distress is a product of intensive residential treatment, as well. It is important to point out, however, that due to the short-term findings, Pennebaker's expressive writing paradigm appears to be practical for use in women with SUDs, as the writing task generates the same immediate responses as it does for other, non-clinical populations.

\section{Expressive Writing with Drug Dependent Women}

This project examined whether expressive writing improved outcomes in a residential-based sample of women with SUDs. Women were able to safely express their deepest emotions and thoughts, many for the first time, about a traumatic and/or stressful event that occurred. Expressive writing was a safe way for them to express and process their feelings. During the follow-up assessments, the majority of women who were in the expressive writing condition reported that the writing helped them work through issues and problems, and deal more effectively with feelings and emotions related to trauma and stress. These reports were offered spontaneously by the participants, was well as when providing responses to the Follow-Up Questionnaire.

The women who present for treatment at Rubicon are somewhat homogenous in the sense that the majority have less than a high school level of education, are underemployed or unemployed, uninsured, single/never married, and thus are a vulnerable population with a variety of medical and psychosocial problems and fewer resources than 
others with SUDs. The study population also represents women of diverse race, substance use diagnoses, and psychiatric symptom severity. It is noteworthy that in a population that generally has difficulty adhering to treatment, expressive writing was well-received and regarded as helpful by the participants themselves.

While findings suggest some loss in ability to see group differences because of the positive changes associated with 30 days of residential drug treatment, we gained in rates of adherence. Furthermore, retention for the intervention and follow-up assessment would likely not have been obtained in an outpatient setting, and the intervention, itself, could have raised safety issues. However, the present study showed a strong retention rate $(94.6 \%$ completed all four writing sessions, $88.6 \%$ completed the two-week followup, and $80.5 \%$ completed the one-month follow-up), which is higher than most other studies of substance dependent populations (Najavits, Weiss, Shaw, \& Muenz, 1998). Women in the expressive writing condition also reported finding the writing to be helpful (98.6\%), and many continued writing on their own (42.0\%) after study completion, using the instructions given during the study.

The present study also provides evidence for the fidelity of the writing intervention in a residential SUD treatment center, as those writing about stressful or traumatic experiences were experiencing the anticipated responses per Essay Evaluation Measures and PANAS scores. Those in the expressive writing condition reported that their essays were more meaningful, personal, and revealing of their emotions compared to the control group. Topics of the expressive writing essays were also written about a 
severe trauma or stressor, and more likely to still be influencing their lives, compared to the control writing condition.

Together, these findings indicate that addressing trauma-related issues concurrently with substance abuse treatment was well-received by study participants. Additionally, women in the expressive writing condition either improved more than, or as much as, those in the control writing condition on measures of physical and psychological health at follow-up. While the lack of adverse events is not a telltale sign of treatment efficacy, it does inform treatment providers that implementing such traumafocused interventions can be done without triggering more harm, and can possibly work to enhance current treatment procedures (Killeen, et al., 2008). Expressive Writing at One Month Follow-Up in a Residential SUD Treatment Program

Since expressive writing research has historically been conducted mostly with healthy college students, and has only recently been extended to include medical and clinical populations, we are still early on in our understanding of how and for whom this paradigm works. Nevertheless, it has been suggested that expressive writing might be less effective for people who indicate very high levels of psychopathology and/or with treatment-seeking samples (Sloan \& Marx, 2004). Further, recent research suggests that high risk individuals may require supplemental interventions (e.g., therapy, traumarelated skills training) in order to obtain maximal benefits from expressive writing (Smyth, Hockemeyer, \& Tulloch, 2008).

Furthermore, while the content of individual essays was not examined in the present paper, it would also be an important issue to consider in facilitating our 
understanding of how to best use the written disclosure procedure as well as what moderators and mediators may exist (Sloan, Marx \& Epstein, 2005). Examining mediators and moderators (Frattaroli, 2006), such as whether the level of disclosure is related to symptom improvements (Brown \& Heimberg, 2001), may help us better understand how and for whom expressive writing works. For example, Brown \& Heimberg (2001), explored the number of words in each essay (to measure the degree of detail) and the number of self-references (to measure the level at which the account was personalized) and found the number of self-referenced words had a curvilinear relationship with symptom reduction among female rape victims when assessed at one month follow-up.

Future analyses should also examine whether essay topic (e.g. same versus different experience across all sessions), vocabulary (e.g., negative emotion words; cause/effect words), or essay descriptiveness (e.g., number of words; self-reference words) were more likely to be related longer-term improvements among a female drug dependent population.

Furthermore, the present findings suggest that the expressive writing paradigm may not have been powerful enough to produce significant benefits above and beyond the intensive residential treatment these women were receiving. This is not to say, however, that without a little tweaking of the paradigm, whether in the number of days spent writing (e.g., 8 days versus 4 days of writing), or a different instructional set for the disclosure condition (e.g., having participants write about the same topic each day; a 
certain type of trauma [e.g., assault]; or the most significant trauma indicated on the PDS, etc.), expressive writing would not have been more effective with this population. Study Implications, Future Directions, and Applications

The present study has a number of important implications. First, this study supports the notion that repeated trauma and PTSD are pervasive among female SUD populations. Results indicate that treatment providers should be aware of the high prevalence of trauma among SUD patients, as women with both trauma and SUDs are likely to have more severe psychological and physical health problems compared to those without trauma and/or PTSD.

While many treatment providers have expressed concern that integrated trauma interventions pose a risk for increasing the individual's psychiatric and physical health symptoms (Hien, et al., 2004), the present study provides evidence that trauma-focused interventions can be incorporated into traditional residential SUD treatment without triggering more harm. This clinical trial also supports the notion that counselors can address both problems simultaneously, with evidence that such an approach actually contributes to addiction recovery, rather than derailing SUD patients from abstinence (Brady, Dansky, Back, Foa, Carroll, 2001; Hien, et al., 2004; Najavits, 2006).

To the best of our knowledge, this study is the first to examine the utility of the expressive writing paradigm with drug dependent women. Furthermore, the findings support compliance to the writing instructions through mood changes that occurred following writing sessions, as well as outcomes from the Essay Evaluation Measure. The study had a high adherence to the treatment, and both writing groups responded to 
instructions as anticipated. Expressive writing participants reported that their writing was more personal, meaningful, and revealing of their emotions than control writing participants.

According to the follow-up questionnaire, the majority of women who were in the expressive writing condition reported that the writing helped them work through issues and problems, and deal more effectively with feelings and emotions related to trauma and stress. Examples of responses (selected randomly) to an open-ended question from the Follow-Up Questionnaire ('Did you find the writing helpful? Why? Why not?'), can be found in Table 14.

Thus, expressive writing was found to be a brief and cost effective adjunct to current residential SUD treatment. Expressive writings' efficacy, efficiency (15-20 minutes per session over four consecutive days), and cost-effectiveness (the cost of a notebook), suggest that writing as a means for disclosing traumatic experiences may be a useful adjunct to traditional alcohol and drug treatment, particularly in SUD treatment settings that do not routinely screen for trauma and PTSD and therefore, do not specifically focus on such issues during treatment. Overall, the present study revealed significant and non-significant improvements across all psychological and physical health measures at the one month follow-up for both writing conditions. Future studies should evaluate whether expressive writing affects SUD women with co-morbid PTSD differently than women without co-morbid PTSD.

While the present findings indicate that expressive writing as a trauma-focused intervention could be implemented in a residential SUD treatment center without 
triggering harm, further investigation is still needed in order to understand how expressive writing can most appropriately be integrated with, or used as an adjunct to, psychotherapeutic techniques currently used in treatment programs.

Furthermore, the present study was conducted in a residential treatment facility, where 24-hour staff was available in the case of any adverse events. While there were no adverse events reported as a result of participating in this study, it is possible that participants felt 'safe' being able to write in a confidential setting, where support was available if need be. The author cautions others about conducting this type of clinical trial in an outpatient SUD treatment setting, where participants could possibly (and likely) go back to an environment where trauma or victimization could occur, especially following the written disclosure task. Therefore, further research is needed to examine if benefits of expressive writing could generalize to SUD patients attending outpatient treatment. A next step might be to conduct an expressive writing study using methadone maintenance treatment patients, where new patients would be required to visit the clinic daily. 
Table 14

Follow-Up Questionnaire: 10 randomly selected Quotes from Each Writing Condition

\begin{tabular}{|c|c|}
\hline $\begin{array}{l}\text { Expressive } \\
\text { Writing } \\
\text { Participants }\end{array}$ & $\begin{array}{c}\text { Question 4: Did you find the writing helpful? Why? Why not? } \\
\text { (please put your answer below) }\end{array}$ \\
\hline & $\begin{array}{l}\text { "Very helpful. I've never in my life experienced something like this. It } \\
\text { hit the core of everything" }\end{array}$ \\
\hline & "Because it helped me express some of my feelings that I held inside" \\
\hline & "It made me realize that what happened to me wasn't my fault" \\
\hline & $\begin{array}{l}\text { "Because it helped me to bring back to surface some of the stuff I had } \\
\text { suppressed" }\end{array}$ \\
\hline & $\begin{array}{l}\text { "Very helpful. It got me in touch with my feelings and helped me } \\
\text { work through some of my issues and problems. It was good for me" }\end{array}$ \\
\hline & $\begin{array}{l}\text { "Made me bring some of the issues that wrote about, especially the } \\
\text { attempted rape and how I was able to not have sex because of fear" }\end{array}$ \\
\hline & $\begin{array}{l}\text { "It helped me share, get out some of my feelings, it's better when } \\
\text { you're writing things" }\end{array}$ \\
\hline & $\begin{array}{l}\text { "Because it's helping me to deal with the things I have gone through. } \\
\text { My mask came down a little bit. Now I don't get teary-eyed as often. } \\
\text { Helped take some off my shoulders" }\end{array}$ \\
\hline & "Very helpful. Helped me to vent everything I've had built up inside" \\
\hline & $\begin{array}{l}\text { "Because it made me think back to some issues that were hindering } \\
\text { me from moving forward. It helps me let go and move forward. I feel } \\
\text { much better" }\end{array}$ \\
\hline Control Writing & "Shallow subject matter" \\
\hline & "It was just being plain, you can't express no emotions" \\
\hline & $\begin{array}{l}\text { "Kind of neutral! Not aware of what the goal was. Helps me in my } \\
\text { purpose in gaining knowledge of it" }\end{array}$ \\
\hline & $\begin{array}{l}\text { "Gave me insight to how much I remember the previous day's } \\
\text { events" }\end{array}$ \\
\hline & "Because it made me realize that I can write just about anything" \\
\hline & $\begin{array}{l}\text { "Not so much writing about food, but just spending that } 20 \text { minutes } \\
\text { writing, I did"" }\end{array}$ \\
\hline & $\begin{array}{l}\text { "It made me think in ways I never thought of and I still think about it } \\
\text { now" }\end{array}$ \\
\hline & "It helped me realize/ remember a lot of things" \\
\hline
\end{tabular}




\begin{tabular}{|l|l|}
\hline & $\begin{array}{l}\text { "I found out that it was kind of hard trying to remember everything I } \\
\text { did in a day's time" }\end{array}$ \\
\hline & "It helped my brain to work so I could remember things" \\
\hline
\end{tabular}

\section{Study Limitations}

The present study had a number of limitations. First, one month is a relatively short follow-up period. It is important that future studies examine group differences over longer periods of time post-intervention, even though other studies show the most dramatic effects more immediately following the intervention. Another limitation of this study is that physical health outcome measures were all based solely on self-report, which has a tendency to be a less reliable and less objective measure of health (Sloan, Marx \& Epstein, 2005). It would have been useful to include objective data such as medical/clinical staff ratings (blind to study condition) as well as absentee rates from Rubicon groups and other activities.

Furthermore, the brief drug craving scale used in this study may have been inadequate in detecting true craving levels, and future studies should incorporate other measures of craving, or post-residential treatment follow-ups in order to determine relative craving and relapse rates once in a non-controlled environment. Moreover, as already pointed out, the present study was conducted in a residential facility and can not be generalized to outpatient SUD patient populations.

As noted earlier, people with more severe mental health problems (e.g., SUDs, PTSD, Depression), may require more writing sessions or longer writing session. It would also be useful to examine potential moderators, including questionnaires assessing avoidance (high avoidance versus low avoidance personalities). There is also evidence 
that expressive writing is more useful with males (Frattaroli, 2006). Thus, it would be important for future research to examine whether gender does in fact impact outcomes, particularly with SUD populations.

Another important consideration when interpreting the findings of the present study is that, although participants received scripted instructions for each writing condition, participants in the expressive writing condition were not required to address any particular experience in their writing. Thus, expressive writing participants could independently choose the topic of disclosure and it could remain constant across the four writing sessions or it could vary across sessions. It would be helpful to determine whether participants wrote about the traumatic events they reported on the PDS questionnaire, such as a particular assault versus something about a death of a parent, for example.

It is important to note that the primary focus of the present study was on short term beneficial or detrimental effects associated with expressive writing, when offered as an adjunct to drug abuse treatment. For safety reasons, this initial RCT was conducted in a residential setting and provided benchmark data on short term outcomes associated with expressive writing. Specifically, the study examined whether expressive writing posed any increased risk for premature treatment drop out or relapse to substance use. Outcome data supported the safety of the experimental paradigm and yielded no evidence of shortterm negative outcomes relative to the control group. Because the safety issues were of central importance, the present study did not systematically examine whether a focus on trauma-related issues early in treatment would also lead to better longer-term outcomes 
(e.g., lower rates of relapse and re-victimization following discharge from residential care). Since present study findings supported the safety of expressive writing as an adjunct to drug abuse treatment, the stage is now set for future studies that include longer-term follow-up. Specifically, subsequent research should track participants postintervention over longer periods of time and particularly following discharge from residential care, with a focus on physical and psychological functioning as well as relapse to drug use.

No specific questions were asked about the traumatic event(s) chosen as targets for expressive writing or the factors that contributed to their selection. Future data analyses will provide more detailed information, with an opportunity to compare essay content with PDS assessment results. The present study elected not to specify how traumatic events should be selected for focus in expressive writing because research todate has yielded mixed results and no studies have focused on women with SUDs. With more general population samples, some research has found beneficial effects regardless of instructions, while others found that writing about specific topics was more beneficial. This is clearly an area that warrants further attention and could prove important in the use of expressive writing as an adjunct to drug abuse treatment. 


\section{References}

Alterman, A. I., Randall, M., \& McClellan, A. T. (2000). Comparison of outcomes by gender and fee-for-services versus managed care: A study of nine community programs. Journal of Substance Abuse Treatment, 19, 127-134.

American Psychiatric Association. (2000). Diagnostic and Statistical Manual of Mental Disorders, Fourth Edition, Text Revision. Washington, DC: American Psychological Association.

Arfken, C.L., Klein, C., Menza, S.D., Schuster, C. R. (2001). Gender differences in problem severity at assessment and treatment retention. Journal of Substance Abuse Treatment, 20, 53-57.

Back, S. E., Brady, B. T., Sonne, S. C., Verduin, M. L. (2006). Symptom improvement in co-occurring PTSD and alcohol dependence. The Journal of Nervous and Mental Disease, 194, 690-696.

Bartels, S. J., \& Thomas, W. N. (1991). Lessons from a pilot residential treatment program for people with dual diagnoses of severe mental illness and substance use disorder. Psychosocial Rehabilitation Journal, 15, 19-30.

Batten, S. V., \& Hayes, S. C. (2005). Acceptance and commitment therapy in the treatment of comorbid substance abuse and post-traumatic stress disorder. Clinical Case Studies, 4, 246-262.

Bennett, L. \& Lawson, M. (1994). Barriers to cooperation between domestic-violence and substance-abuse programs. Families in Society: The Journal of Contemporary Human Services, 75, 277-296.

Boles, S. M., Joshi, V., Grella, C., \& Wellisch, J. (2005). Childhood sexual abuse patterns, psychosocial correlates, and treatment outcomes among adults in drug abuse treatment. Journal of Child Sexual Abuse, 14, 39-55.

Booth, R.J., Petrie, K.J., Pennebaker, J.W. (1997). Changes in circulating lymphocyte numbers following emotional disclosure: Evidence or buffering? Stress Medicine, $13,23-29$.

Brady, K. T., Grice, D. E., Dustan, L., \& Randall, C. (1993). Gender differences in substance use disorders. American Journal of Psychiatry, 150, 1707-1711.

Brady, K. T., Killeen, T., Saladin, M. E., Dansky, B., \& Becker, S. (1994). Comorbid substance abuse and posttraumatic stress disorder: Characteristics of women in 
treatment. American Journal on Addictions, 3, 160-163.

Brady, K. T., Dansky, B. S., Sonne, S. C., \& Saladin, M. E. (1998). Posttraumatic stress disorder and cocaine dependence. American Journal on Addictions, 7, 128-135.

Brady, K. T., Dansky, B., Back, S., Foa, E., \& Carroll, K. (2001). Exposure therapy in the treatment of PTSD among cocaine-dependent individuals: Preliminary findings. Journal of Substance Abuse Treatment, 21, 47-54.

Breslau, N. (2001). Outcomes of Posttraumatic Stress Disorder. Journal of Clinical Psychiatry, 62 (Suppl 17), 55-59.

Briere, J. N., \& Conte, J. (1993). Self-reported amnesia for abuse in adults molested as children. Journal of Traumatic Stress, 6, 21-31.

Brown, P. J., \& Wolfe, J. (1994). Substance abuse and post-traumatic stress disorder comorbidity. Drug and Alcohol Dependence, 35, 51-59.

Brown, P. J., Recupero, P. R., Stout, R. (1995). PTSD substance use comorbidity and treatment utilization. Addictive Behaviors, 20, 251-254.

Brown, P. J., Stout, R. L., \& Mueller, T. (1996). Post-traumatic stress disorder and substance abuse relapse among women: A pilot study. Psychology of Addictive Behaviors, 10, 124-128.

Brown, P. J., Stout, R. L., \& Mueller, T. (1999). Substance use disorder and posttraumatic stress disorder comorbidity: Addiction and psychiatric treatment rates. Psychology of Addictive Behaviors, 13, 115-122.

Brown, E.J., Heimberg, R.G. (2001). Effects of Writing about Rape: Evaluating Pennebaker's Paradigm with a Severe Trauma. Journal of Traumatic Stress, 14, 781790.

Boyd, C. J., Hill, E., Holmes, C. \& Purnell, R. (1998). Putting drug use in context: Lifelines of African American women who smoke crack. Journal of Substance Abuse Treatment, 1998, 235-249.

Campbell, N. B. (2003). Emotional disclosure through writing: An intervention to facilitate adjustment to having a child with autism (Doctoral dissertation, University of Mississippi, 2003). Dissertation Abstracts International, 64, 2380.

Chilcoat, H. D. \& Breslau, N. (1998). Posttraumatic stress disorder and drug disorders: Testing causal pathways. Archives of General Psychiatry, 55, 913-917. 
Clark, H. W., Masson, C. L., Delucchi, K. L., Hall, S. M., \& Sees, K. L. (2001). Violent traumatic events and drug abuse severity. Journal of Substance Abuse Treatment, 20, 121-127.

Coffey, S. F., Dansky, B. S., Falsetti, S. A., Saladin, M. E., \& Brady, K. T. (1998). Screening for PTSD in a substance abuse sample: Psychometric properties of a modified version of the PTSD Symptom Scale self-report. Journal of Traumatic Stress, 11, 393-399.

Coffey, S. F., Saladin, M. E., Drobes, D. J., Brady, K. T., Dansky, B. S., \& Kilpatrick, D. G. (2002). Trauma and substance cue reactivity in individuals with comorbid posttraumatic stress disorder and cocaine or alcohol dependence. Drug and Alcohol Dependence, 65, 115-127.

Coffey, S. F., Schumacher, J. A., Brimo, M. L., \& Brady, K. T. (2005). Exposure therapy for substance abusers with PTSD: Translating research to practice. Behavior Modification, 29, 10-38.

Cohen, F. S., \& Densen-Gerber, J. (1982). A study of the relationship between child abuse and drug addiction in 178 patients: Preliminary results. Child Abuse and Neglect, 6, 383-387.

Cohen, J., Cohen, P., West, S.G., Aiken, L.S. (2003). Applied multiple regression/correlation analysis for the Behavioral sciences. New Jersey: Erlbaum Associates.

Cohen, L. R., \& Hien, D. A. (2006). Treatment outcomes for women with substance abuse and PTSD who have experienced complex trauma. Psychiatric Services, $57,100-106$.

Copeland, J. (1997). A qualitative study of barriers to formal treatment among women who self-managed change in addictive behaviours. Journal of Substance Abuse Treatment, 14, 198-190.

Cottler, L. B., Compton, W. M., Mager, D., Spitznagel, E. L., \& Janca, A. (1992). Posttraumatic stress disorder among substance abusers from a general population. American Journal of Psychiatry, 149, 664-670.

Da Silveira, D. X., Doering-Silveira, E., Niel, M., \& Jorge, M. R. (2006). Predicting craving among cocaine users. Addictive Behaviors, 31, 2292-2297.

Dansky, B. S., Saladin, M E, Brady, K T, Kilpatrick, D. G., \& Resnick, H. S. (1995). Prevalence of victimization and posttraumatic stress disorder among women with substance use disorders: Comparison of telephone and in-person assessment 
samples. International Journal of the Addictions, 30, 1079-1099.

Dansky, B. S., Brady, K. T., Saladin, M. E., Killeen, T., Becker, S., \& Roitzsch, J. (1996). Victimization and PTSD in individuals with substance use disorders: Gender and racial differences. American Journal of Drug and Alcohol Abuse, 22, 75-93.

Dansky, B. S., Brady, K. T., \& Saladin, M. E. (1998). Untreated symptoms of PTSD among cocaine-dependent individuals: Changes over time. Journal of Substance Abuse Treatment, 15, 499-504.

Dansky, B. S., Byrne, C. A., \& Brady, K. T. (1999). Intimate violence and posttraumatic stress disorder among individuals with cocaine dependence. American Journal of Drug and Alcohol Abuse, 25, 752-757.

Derogatis, L. R. (1979). Brief Severity Index (BSI). Minneapolis, MN: Pearson, Inc.

Derogatis, L. R., \& Melisaratos, N. (1983). The Brief Symptom Inventory: An introductory report. Psychological Medicine, 13, 595-605.

Desai, S., Arias, L., Thompson, M. P., \& Basile, K. C. (2002). Childhood victimization and subsequent adult revictimization assessed in a nationally representative sample of women and men. Violence and Victims, 17, 639-653.

Esterling, B. A., L’Abate, L., Murray, E. J., \& Pennebaker, J. W. (1999). Empirical foundations for writing in prevention and psychotherapy: Mental and physical health outcomes. Clinical Psychology Review, 19, 79-96.

First, M. B., Spitzer, R. L., Gibbon M., \& Williams, J. B.W.: Structured Clinical Interview for DSM-IV-TR Axis I Disorders, Research Version, Patient Edition. (SCID-I/P) New York: Biometrics Research, New York State Psychiatric Institute, November 2002.

Foa, E., B., Riggs, D. S., Dancu, C. B., \& Rothbaum, B. O. (1993). Reliability and validity of a brief instrument for assessing post-traumatic stress fisorder. Journal of Traumatic Stress, 6, 459-473. In P. Ouimette \& P. J. Brown (Eds.), Trauma and substance abuse: Causes, consequences, and treatment of comorbid disorders. Washington, DC: American Psychological Association.

Foa, E. B. (1996). Posttraumatic Stress Diagnostic Scale. Minneapolis, MN: National Computer Systems.

Foa, E. B. (1997). Psychological processes related to recovery from a trauma and an 
effective treatment for PTSD. Annals of the New York Academy of Sciences, 821, 410-424.

Foa, E. B., Cashman, L., Jaycox, L., \& Perry, K. (1997). The validation of a self-report measure of Posttraumatic Stress Disorder: The posttraumatic diagnostic scale. Psychological Assessment, 9, 445-451.

Foa, E. B., \& Street, G. P. (2001). Women and traumatic events. Journal of Clinical Psychiatry, 62, 29-34.

Francis, M. E., \& Pennebaker, J. W. (1992). Putting stress into words: The impact of writing on physiological, absentee, and self-reported emotional well-being measures. Stress Management, 6, 280-287.

Frattaroli, J. (2006). Experimental disclosure and its moderators: A meta-analysis. Psychological Bulletin, 132, 823-865.

Frisina, P. G., Borod, J. C., \& Lepore, S. J. (2004). A meta-analysis of the effects of written emotional disclosure on the health outcomes of clinical populations. The Journal of Nervous and Mental Disease, 192, 629-634.

Fullilove, M.P., Fullilove, R. E., Smith, M., Winkler, K., Michael, C., Panzer, P.G., et al. (1993). Violence, trauma, and posttraumatic stress disorder among women drug users. Journal of Traumatic Stress, 6, 533-543.

Gatz, M., Russell, L. A., Grady, J., Kram-Fernandez, D., Clark, C., \& Marshall, B. (2005). Women's recollections of victimization, psychological problems, and substance abuse. Journal of Community Psychology, 33, 479-493.

Garavan, H., Pankiewicz, J., Bloom, A., Cho, J.K., Sperry, L., Ross, T.J., Salmeron, B. J., Risinger, R., Kelley, D., \& Stein, E.A. (2000). Cue-induced cocaine craving: neuroanatomical specificity for drug users and drug stimuli. The American Journal of Psychiatry, 157, 1789-1798.

Gerstein, D. R., \& Johnson, R. A. (2000). Characteristics, services, and outcomes of treatment for women. Journal of Psychopathological Behavior Assessment, 22, 325-338.

Gilbert, L., El-Bassel, N., Schilling, R. F., \& Friedman, E. (1997). Childhood abuse as a risk for partner abuse among women in methadone maintenance. American Journal on Drug and Alcohol Abuse, 23, 581-595.

Greenberg, M. A., \& Stone, A. A. (1992). Emotional disclosure about traumas and its relation to health: Effects of previous disclosure and trauma severity. Journal of 
Personality and Social Psychology, 63, 75-84.

Greenfield, S. F., Trucco, E. M., McHugh, R. K., Lincoln, M., \& Gallop, R. J. (2007). The women's recovery group study: A stage I trial of women-focused group therapy for substance use disorders versus mixed-gender group drug counseling. Drug Alcohol Dependence, doi 10.1016, 1-9.

Grella, C. E., Scott, C. K., Foss, M. A., Joshi, V., \& Hser, Y-I. (2003). Gender differences in drug treatment outcomes among participants in the Chicago Target Cities Study. Evaluation and Program Planning, 26, 297-310.

Grice, D. E., Brady, K. T., Dustan, L. R., Malcolom, R., \& Kilkpatrick, D. G. (1995). Sexual and physical assault history and posttraumatic stress disorder in substancedependent individuals. The American Journal on Addictions, 4, 297-305.

Grove, W. M., Eckert, E. D., Heston, L., Bouchard, T. J., Segal, N., \& Lykken, D. T. (1990). Heritability of substance abuse and antisocial behavior: a study of monozygotic twins reared apart. Biological Psychiatry, 27, 1293-1304.

Gutierres, S. E., \& Todd, M. (1997). The impact of childhood sexual abuse on treatment outcomes of substance abusers. Professional Psychology: Research and Practice, $28,348-354$.

Gutierres, S. E., Van Puymbroeck, C. V. (2006). Childhood and adult violence in the lives of women who misuse substances. Aggression and Violent Behavior, 11, 497-513.

Hien, D. A., Cohen, L. R., Miele, G. M., Litt, L. C., \& Capstick, C. (2004). Promising treatments for women with comorbid PTSD and substance use disorders. American Journal of Psychiatry, 161, 1426-1432.

Hien, D., Cohen, L., \& Campbell, A. (2005). Is traumatic stress a vulnerability factor for women with substance use disorders? Clinical Psychology Review, 25, 813-823.

http://www.liwc.net/liwcdescription.php\#index4

Ireland, T., \& Widom, C. S. (1994). Childhood victimization and risk for alcohol and drug arrests. International Journal of the Addictions, 29, 235-274.

Jacobsen, L. K., Southwick, S. M., \& Kosten, T. R. (2001). Substance use disorders in patients with posttraumatic stress disorder: A review of the literature. American Journal of Psychiatry, 158, 1184-1190.

Jang, K. L., Stein, M. B., Taylor, S., Asmundson, G. J., Livesley, W. J. (2003). 
Exposure to traumatic events and experiences: aetological relationships with personality function. Psychiatry Research, 30, 61-69.

Johnson, S. D., Striley, C., Cottler, L. B. (2006). The association of substance use disorders with trauma exposure and PTSD among African American drug users. Addictive Behaviors, 31, 2063-2073.

Kallay, E., Vaida, S., Borla, S., \& Opre, A. (2008). The benefits of classic and enhanced tasks of expressive writing for the emotional life of female freshman students: A pilot study. Cognition, Brain, Behavior, 2008, 251-264.

Kendler, K. S., Bulik, C. M., Silberg, J. Hettema, J. M., Meyers, J, \& Prescott, C. A. (2000). Childhood sexual abuse and adult psychiatric and substance use disorders in women: An epidemiologic and co-twin control analysis. Archives of General Psychiatry, 57, 953-959.

Kessler, R. C., Sonnega, A., Bromet, E., Hughes, M., \& Nelson, C. B. (1995). Posttraumatic stress disorder in the National Comorbidity Survey. Archives of General Psychiatry, 52, 1048-1060.

Kendler, K. S., Prescott, C. A., Neale, M. C., Pedersen, N. L. (1997). Temperance broad registration for alcohol abuse in a national sample of Swedish male twins, born 1902 to 1949. Archives of General Psychiatry, 54, 178-184.

Kernan, W. N., Viscoli, C. M., Makuch, R. W. Brass, L. M., \& Horwitz, R. I. (1999). Stratified Randomization for Clinical Trials. Journal of Clinical Epidemiology, 52, 19-26.

Khantzian, E. F. (1985). The self-medication hypothesis of addictive disorders: Focus on heroin and cocaine dependence. American Journal of Psychiatry, 142, 12591264.

Khantzian, E. J., \& Schneider, R. J. (1986). Treatment implications of a psychodynamic understanding of opioid addicts. In R. E. Meyer (Ed.), Psychopathology and Addictive Disorders. New York: Guilford.

Killeen, T., Hien, D., Campbell, A., Brown, C., Hansen, C., Jianh, H., Kristman-Valente, A., et al. (2008). Adverse events in an integrated trauma-focused intervention for women in community substance abuse treatment. Journal of Substance Abuse Treatment, 35, 304-311.

Kilpatrick, D. G., Acierno, R., Resnick, H. S., Saunders, B. E., \& Best, C. L. (1997). A 
2-year longitudinal analysis of the relationship between violent assault and substance use in women. Journal of Consulting and Clinical Psychology, 65, 834-847.

Kloss, J. D., \& Lisman, S. A. (2002). An exposure-based examination of the effects of written emotional disclosure. British Journal of Health Psychology, 7, 31-46.

Koopman, C., Ismailji, T., Holmes, D., Classen, C. C., Palesh, O., Wales, T. (2005). The effects of expressive writing on pain, depression and posttraumatic stress disorder symptoms in survivors of intimate partner violence. Journal of Health Psychology, 10, 211-221.

Larson, M. J., Miller, L., Becker, M., Richardson, E., Kammerer, N., Thom, J., Gampel, J., \& Savage, A. (2005). Physical health burdens of women with trauma histories and co-occurring substance abuse and mental disorders. The Journal of Behavioral Health Services \& Research, 32, 128-140.

Lepore, S. J. (1997). Expressive writing moderates the relation between intrusive thoughts and depressive symptoms. Journal of Personality \& Social Psychology, $73,1030-1037$.

Lepore, S. J., \& Smyth, J. M. (2002). The writing cure: how expressive writing promotes health and emotional well-being. Washington D.C.: American Psychological Association.

Lepore, S. J., Greenberg, M. A., Bruno, M., \& Smyth, J. M. (2002). Expressive writng and health: Self-regulation of emotion-related experiences, physiology, and behavior. In S. J. Lepore and J. M. Smyth (Eds.), The writing cure: How expressive writing promotes health and emotional well-being. Washington, DC: American Psychological Association.

Low, C. A., Stanton, A. L., \& Danoff-Burg, S. (2006). Expressive disclosure and benefit finding among breast cancer patients: Mechanisms for positive health effects. Health Psychology, 25, 181-189.

Lynch, W. J., Roth, M. E., \& Carroll, M. E. (2002). Biological basis of sex differences in drug abuse: preclinical and clinical studies. Psychopharmacology, 164, 121137.

McGuire, K. M., Greenberg, M. A., \& Gevirtz, R. (2005). Autonomic effects of expressive writing in individuals with elevated blood pressure. Journal of Health Psychology, 10, 197-209.

Miller, B. A., Downs, W. R., \& Testa, M. (1993). Interrelationships between 
victimization experiences and women's alcohol/drug use. Journal of Studies on Alcohol, Supp 11, 109-117.

Morrisey, J. P., Jackson, E. W., Ellis, A. R., Amaro, H., Brown, V. B., \& Najavits, L. M. (2005). Twelve-month outcomes of trauma-informed interventions for women with co-occurring disorders. Psychiatric Services, 56, 1213-1222.

Najavits, L. M., Weiss, R. D., Shaw, S. R. (1997). The link between substance abuse and posttraumatic stress disorder in women: A research review. American Journal on Addictions, 6, 273-283.

Najavits, L. M., Gastfriend, D. R., Baber, J. P., Reif, S., Muenz, L. R., Blaine, J., Frank, A., et al. (1998). Cocaine dependence with and without PTSD among subjects in the National Institute on Drug Abuse Collaborative Cocaine Treatment Study. American Journal of Psychiatry, 155, 214-219.

Najavits, L. M., Weiss, R. D., Shaw, S. R. (1999). A clinical profile of women with posttraumatic stress disorder and substance dependence. Psychology of Addictive Behaviors, 13, 98-104.

Najavits, L. M. (2006). Safety seeking: Therapy for posttraumatic stress disorder and substance use disorder. In V. M. Follette \& J. I. Ruzek. (Eds.), Cognitive Behavioral Therapies for Trauma (pp. 228-257). New York: Guilford Press.

Niv, N., \& Hser, Y-I. (2007). Women-only and mixed gender drug abuse treatment programs: Service needs, utilization, and outcomes. Drug and Alcohol Dependence, 87, 194-201.

Norman, S. A., Lumley, M. A., Dooley, J. A., \& Diamond, M. P. (2004). For whom does it work? Moderators of the effects of written emotional disclosure in a randomized trial among women with chronic pelvic pain. Psychosomatic Medicine, 66, 174-183.

North, C. S., Thompson, S. J., Smith, E. M., \& Kyburz, L. M. (1996). Violence in the lives of homeless mothers in a substance abuse treatment program: A descriptive study. Journal of Interpersonal Violence, 11, 234-249.

Ouimette, P. C., Wolfe, J., \& Chrestman, K. R. (1996). Characteristics of posttraumatic stress disorder-alcohol abuse comorbidity in women. Journal of Substance Abuse, 8, 335-346.

Ouimette, P. C., Ahrens, C., Moos, R. H., Finney, J. W. (1997). Posttraumatic stress disorder in substance abuse patients: relationships 1-year post-treatment outcomes. Psychology of Addictive Behaviors, 11, 34-47. 
Ouimette, P. C., Brown, P. J., Najavits, L. M. (1998). Course and treatment of patients with both substance use and posttraumatic stress disorders. Addictive Behaviors, 23, 785-795.

Ouimette, P. C., Finney, J. W., Moos, R. H., (1999). Two-year Posttreatment functioning and coping of substance abuse patients with posttraumatc stress disorder. Psychology of Addictive Behaviors, 13, 105-114.

Ouimette, P. C., Moos, R. H., \& Finney, J. W. (2003). PTSD treatment and 5-year remission among patients with substance use and posttraumatic stress disorders. Journal of Consulting and Clinical Psychology, 71, 410-414.

Ouimette, P., Goodwin, E., \& Brown, P. J. (2006). Health and well being of substance use disorder patients with and without posttraumatic stress disorder. Addictive Behaviors, 31, 1415-1423.

Paranjape, A., Heron, S., Thompson, M., Bethea, K., Wallace, T., \& Kaslow, N. (2007). Are alcohol problems linked with an increase in depressive symptoms in abused, inner-city African American women? Women's Health Issues, 17, 37-43.

Pelissier, B. \& Jones, N. (2005). A review of gender differences among substance abusers. Crime \& Delinquency, 51, 343-372.

Pennebaker, J. W. (1982). The psychology of physical symptoms. New York: SpringerVerlag.

Pennebaker, J. W., \& Beall, S. K. (1986). Confronting a traumatic events: Toward an understanding of inhibition and disease. Journal of Abnormal Psychology, 95, 274-281.

Pennebaker, J.W., Hughes, C.F., O'Heeron, R.C. (1987). The psychophysiology of confession: linking inhibitory and psychosomatic processes. Journal of Personality and Social Psychology, 52, 781-793.

Pennebaker, J.W., Kiecolt-Glaser, J., Glaser, R. (1988). Disclosure of traumas and immune function: Health Implications for psychotherapy. Journal of Consulting and Clinical Psychology, 56, 239- 245.

Pennebaker, J. W. (1989). Confession, inhibition, and disease. In L. Berkowitz (Ed.), Advances in experimental social psychology, Vol. 22 (pp. 211-244). San Diego, CA: Academic Press.

Pennebaker, J.W., Barger, S.D., Tiebout, J. (1989). Disclosure of traumas and health 
among holocaust survivors. Psychosomatic Medicine, 51, 577-589.

Pennebaker, J.W., Colder, M., Sharp, L.K. (1990). Accelerating the coping process. Journal of Personality and Social Psychology, 58, 528-537.

Pennebaker, J. W. (1993). Putting stress into words: Health, linguistic \& therapeutic implications. Behavior Research and Therapy, 31, 539-548.

Pennebaker, J.W. (1997). Writing about emotional experiences as a therapeutic process. Psychological Science, 8, 162-166.

Pennebaker, J.W., Mayne, T.J., Francis, M.E. (1997). Linguistic predictors of adaptive bereavement. Journal of Personality and Social Psychology, 72, 863-871.

Pennebaker, J.W. \& Seagal, J. (1999). Forming a story: The health benefits of narrative. Journal of Clinical Psychology, 55, 1243-1254.

Pennebaker, J. W. \& Keough, K. A. (1999). Revealing, organizing, and reorganizing the self in response to stress and emotion. (pp. 101-121). In Self, Social Identity, and Physical Health. Contrada, R. J., \& Ashmore, R. D., (Eds.), Oxford University Press, Inc: New York.

Pennebaker, J. W., Francis, M. E., \& Booth, R. J. (2001). Linguistic Inquiry and Word Count (LIWC): LIWC2001 Manual. Mahwah, NJ: Erlbaum Publishers.

Pennebaker, J. W., \& Chung, C. K. (in press). Expressive writing, emotional upheavals, and health. In H. Friedman \& R. Silver (Eds.), Handbook of health psychology. New York: Oxford University Press.

Petrie, K. J., Fontanilla, I., Thomas, M. G., Booth, R. J., \& Pennebaker, J. W. (2004). Effect of written emotional expression on immune function in patients with human immunodeficiency virus infection: A randomized trial. Psychosomatic Medicine, 66, 272-275.

Pickens, R. W., Svikis, D. S., McGue, M., Lykken, D. T., Heston, L. L., Clayton, P. J. (1991). Heterogeneity in the inheritance of alcoholism: a study of male and female twins. Archives of General Psychiatry, 48, 19-28.

Radloff, L. S. (1977). The CES-D scale: A self-report depression scale for research in the general population. Applied Psychological Measure, 1, 285-401.

Read, J. P., Bollinger, A. R., \& Sharkansky, E. (2003). Assessment of comorbid substance use disorder and posttraumatic stress disorder. In P. Ouimette \& P. J. Brown (Eds.), Trauma and substance abuse: Causes, consequences, and 
treatment of comorbid disorders. Washington, DC: American Psychological Association.

Reich, J., Lyons, M., \& Cai, B. (1996). Familial vulnerability factors to post-traumatic stress disorder in military veterans. Acta Psychiatrica Scandinavica, 93, 105-112.

Reynolds, M., Mezey, G., Chapman, M., Wheeler, M., Drummand, C., \& Baldacchino, A. (2005). Co-morbid post-traumatic stress disorder in a substance misusing clinical population. Drug and Alcohol Dependence, 77, 21-258.

Richards, J. M., Beal, W. E., Seagal, J. D., \& Pennebaker, J. W. (2000). Effects of disclosure of traumatic events on illness behavior among psychiatric prison inmates. Journal of Abnormal Psychology, 109,156-160.

Roth, S., Newman, E., Pelcovitz, D., van der Kolk, B., Mandel, F. S. (1997). Complex PTSD in victims exposed to sexual and physical abuse: results from the DSM-IV field trial for posttraumatic stress disorder. Journal of Traumatic Stress, 10, 539555.

Saladin, M. E., Brady, K. T., Dansky, B. S., \& Kilpatrick, D. G. (1995). Understanding comorbidity between PTSD and substance use disorders: Two preliminary investigations. Addictive Behaviors, 20, 643-655.

Salomon, A., Bassuk, S. S., \& Hintington, N. (2002). The relationship between intimate partner violence and the use of addictive substances in poor and homeless single mothers. Violence Against Women, 8, 785-815.

Schwartz, L., \& Drotar, D. (2004). Effects of written emotional disclosure on caregivers of children and adolescents with chronic illness. Journal of Pediatric Psychology, 29, 105-118.

Schuck, A. M., \& Widom, C. S. (2001). Childhood victimization and alcohol symptoms in females: Causal inferences and hypothesized mediators. Child Abuse \& Neglect, 25, 1069-1092.

Sheridan, M. J. (1996). Comparisons of the life experiences and personal functioning of men and women in prison. Families in Society: The Journal of Contemporary Human Services, 77, 423-434.

Slatcher, R.B. \& Pennebaker, J. W. (2006). How do I love thee? Let me count the words: The social effects of expressive writing. Psychological Science, 17, 660664.

Sloan, D.M., \& Marx, B.P. (2004) a. Taking pen to hand: evaluating theories underlying 
the written disclosure paradigm. Clinical Psychology: Science and Practice, 11, 121-137.

Sloan, D.M., \& Marx, B.P. (2004) b. A closer examination of the structured written disclosure procedure. Journal of Consulting and Clinical Psychology, 72, 165-175.

Sloan, D. M., Marx, B. P., \& Epstein, E. M. (2005). Further examination of the exposure model underlying the efficacy of written emotional disclosure. Journal of Consulting and Clinical Psychology, 73, 549-554.

Sloan, D. M., Marx, B. P., Epstein, E. M., \& Lexington, J. M. (2007). Does altering the writing instructions influence outcome associated with written disclosure? Behavior Therapy, 38, 155-168.

Smith, M., \& Glass, G. (1977). Meta-analysis of psychotherapy outcome studies. American Psychologist, 32, 752-760.

Smith, S., Anderson-Hanley, C., Langrock, A., \& Compas, B. (2005). The effects of journaling for women with newly diagnosed breast cancer. Psycho-oncology, 14, 1075-1082.

Smyth, J.M. (1998). Written emotional expression: Effect sizes, outcome types, and moderating variables. Journal of Consulting and Clinical Psychology, 66, 170184.

Smyth, J. M. (1999). Written disclosure: Evidence, potential mechanism, and potential treatment. Advances in Mind-Body Medicine, 15, 161-195.

Smyth, J. M., Stone, A. A., Hurewitz, A., \& Kaell, A. (1999). Effects of writing about stressful experiences on symptom reduction in patients with asthma or rheumatoid arthritis. Journal of the American Medical Association, 281, 1304-1309.

Somoza, E., Baker, S., Himmler, C., LoCastro, J., \& Mezinskis, J., Simon, S. et al. (1999). The Brief Substance Craving Scale - measuring craving in clinical trials. NIDA Research Monograph, 180, 304.

Spera, S. P., Buhrfeind, E. D., \& Pennebaker, J. W. (1994). Expressive writing and coping with job loss. Academy of Management Journal, 37, 722-733.

Stanton, A. L., Danoff-Burg, S., Sworowski, L. A., Collins, C. A., Brandstetter, A. D., Rodriguez-Hanley, A., et al. (2002). Randomized, controlled trial of written emotional expression and benefit finding in breast cancer patients. Journal of Clinical Oncology, 11, 93-102. 
Stasiewicz, P. R., \& Maisto, S. A. (1993). Two-factor avoidance theory: the role of negative affect in the maintenance of substance use and substance use disorders. Behavior Therapy, 24, 337-356.

Stein, M. B., Jang, K. L., Taylor, S., Vernon, P. A., \& Livesley, W. J. (2002). Genetic and environmental influences on trauma exposure and posttraumatic stress disorder symptoms: a twin study. The American Journal of Psychiatry, 159, $1675-1681$.

Stewart, S. H. (1996). Alcohol abuse in individuals exposed to trauma: A critical review. Psychological Bulletin, 120, 83-112.

Stewart, S. H., Ouimette, P., \& Brown, P. J. (2002). Gender and the comorbidity of PTSD with substance use disorders. In R. Kimberling, \& P. Ouimette (Eds.), Gender and PTSD (pp. 232-270). New York, NY: Guilford Press.

Stewart, S. H., Grant, V. P., Ouimette, P., \& Brown, P. J. (2006). Are gender differences in post-traumatic stress disorder rates attenuated in substance use disorder patients? Canadian Psychology, 47, 110-124.

Substance Abuse and Mental Health Services Administration. (2000). Summary of findings from the 1999 National Household Survey on Drug Abuse (DHHS Publication No. 00-3466). Washington, DC: Government Printing Office.

Testa, M., Livingston, J. A., \& Leonard, K. E. (2003). Women's substance use and experiences of intimate partner violence: A longitudinal investigation among a community sample. Addictive Behaviors, 28, 2649-1664.

Turner, W. L. (2000). Cultural considerations in family-based primary prevention programs in drug abuse. Journal of Primary Prevention, 21, 285-303.

Turner, W. L. \& Wallace, B. (2003). African American substance use: Epidemiology, prevention, and treatment. Violence Against Women, 9, 576-589.

Van Etten, M. L., \& Anthony, J. C. (2001). Male-female differences in transitions from first drug use opportunity to first use: Searching for subgroup variation by age, race, region, and urban status. Journal of Women's Health \& Gender-Based Medicine, 10, 797-804.

Watson, D., Clark, L. A., \& Tellegen, A. (1988). Development and validation of brief measures of positive and negative affect: The PANAS scales. Journal of Personality and Social Psychology, 54, 1063-1070.

Weathers, F. W., Litz, B. T., Herman, D. S., Huska, J. A., \& Keane, T. M. (1993, 
October). The PTSD Checklist (PCL): Reliability, validity, and diagnostic utility. Paper presented at the annual meeting of the International Society for Traumatic Stress Studies, San Antonio, TX. In P. Ouimette \& P. J. Brown (Eds.), Trauma and substance abuse: Causes, consequences, and treatment of comorbid disorders. Washington, DC: American Psychological Association.

Woodhouse, L. (1992). Women with jagged edges: Voices from a culture of substance abuse. Qualitative Health Research, 2, 262-281.

Xian, H., Chantarujikapong, S. I., Scherrer, J. F., Eisen, S. A., Lyons, M.J., Goldberg, J., Tsuang, M., True, W. R. (2000). Genetic and environmental influences on posttraumatic stress disorder, alcohol and drug dependence in twin pairs. Drug and Alcohol Dependence, 61, 95-102.

Zettle, R. D., Petersen, C. L., Hocker, T. R., \& Provines, J. L. (2007). Responding to a perceptual motor task as a function of level of experiential avoidance. The Psychological Record, 57, 49-62. 


\section{Appendix A}

\section{Overview of writing instructions given to experimental condition}

This study is an extremely important project looking at writing. Over the next four days, you will be asked to write about one of several different topics for 20 minutes each day. You will first meet with me at this office and I will give you your instructions. You will then be escorted to a comfortable room where you will not be disturbed (e.g., you room) and will be alone to write. The person who brings you to the room will close the door: that will be your signal to begin writing. At the end of 20 minutes, the person will knock on your door to let you know that the 20 minutes are up.

The only rule we have about your writing is that you write continuously for the entire time. If you run out of things to say, just repeat what you have already written. In your writing, don't worry about grammar, spelling, or sentence structure. Just write. Different people will be asked to write about different topics. Because of this, I ask that you not talk with anyone about the experiment. Because we are trying to make this a tight experiment, I can't tell you what other people are writing about or anything about the nature or predictions of the study. Once the study is complete, however, we will tell you everything. Another thing is that some people feel a little sad or depressed after writing. If that happens, it is completely normal. Most people say that these feelings go away in an hour or so. If at any time over the course of the experiment you feel upset or distressed, please contact Dr. Dace Svikis or Sarah Meshberg-Cohen immediately [Note: All participants will receive a sheet with contact information for Dr. Svikis and Ms. Meshberg-Cohen].

Another thing. Your writing is completely anonymous and confidential. Your booklet is coded with an ID number. Please do not write your name on the booklet. I promise that none of the experimenters, including me, will link your writing to you. The one exception is that if your writing indicates that you intend to harm yourself or others, we are legally bound to match your ID with your name. Above all, we respect your privacy. Do you have any questions at this point? Do you still wish to participate?

\section{Experimental Condition Instructions}

Over the next four days, I want you to write about your deepest emotions and thoughts about the most traumatic experience in your life. In your writing, I'd like you to really let go and explore your very deepest emotions and thoughts. You might tie this trauma to your childhood, your relationship with others, including your parents, lovers, friends, or relatives. You may also link this event to your past, present, or your future, or to who you have been, who you would like to be, or who you are now. You may write about the same general issues or experiences on all days of writing or on different topics each day. Not everyone has had a single trauma but all of us have had major conflicts or stressors in our lives and you can write about them as well. All of your writing will be completely confidential. Don't worry about spelling, sentence structure, punctuation or grammar. The only rule is that once you begin writing, continue to do so until your time is up. 


\section{On the second day of writing}

How did yesterday's writing go? Today, I want you to continue writing about the most traumatic experience in your life. It could be the same topic that you wrote about yesterday, or it could be something different. But today, I really want you to explore your very deepest emotions and thoughts...

\section{On the third day of writing}

In your writing today, I again want you to explore your deepest thoughts and feelings about the most traumatic experience of your life. It could be the same topic that you wrote about yesterday, or it could be something different. But today, I really want you to explore your very deepest emotions and thoughts...

\section{On the fourth day of writing}

Today is the last writing session. In your writing today, I again want you to explore your deepest thoughts and feelings about the most traumatic experience of your life.

Remember that this is the last day and so you might want to wrap everything up. For example, how is this experience related to your current life and your future? But feel free to go in any direction you feel most comfortable with and delve into your deepest emotions and thoughts... 


\section{Overview of writing instructions given to control condition}

This study is an extremely important project looking at writing. Over the next four days, you will be asked to write about one of several different topics for 20 minutes each day. You will first meet with me at this office and I will give you your instructions. You will then be escorted to a comfortable room where you will not be disturbed (e.g., you room) and will be alone to write. The person who brings you to the room will close the door: that will be your signal to begin writing. At the end of 20 minutes, the person will knock on your door to let you know that the 20 minutes are up.

The only rule we have about your writing is that you write continuously for the entire time. If you run out of things to say, just repeat what you have already written. In your writing, don't worry about grammar, spelling, or sentence structure. Just write. Different people will be asked to write about different topics. Because of this, I ask that you not talk with anyone about the experiment. Because we are trying to make this a tight experiment, I can't tell you what other people are writing about or anything about the nature or predictions of the study. Once the study is complete, however, we will tell you everything.

Another thing. Your writing is completely anonymous and confidential. Your booklet is coded with an ID number. Please do not write your name on the booklet. I promise that none of the experimenters, including me, will link your writing to you. The one exception is that if your writing indicates that you intend to harm yourself or others, we are legally bound to match your ID with your name. Above all, we respect your privacy. Do you have any questions at this point? Do you still wish to participate?

\section{Control Condition Instructions}

Over the next four days, I will give you a writing assignment. In today's writing, I would like you to describe what you have eaten since yesterday morning. I am not interested in your emotions or opinions. Rather I want you to write about specific objects or events in detail without discussing your thoughts or feelings. Feel free to be as detailed as possible. Again, I want you to describe exactly what you have eaten up until coming to this experiment. For example, you might describe the texture, the color, an estimate of calories and fat, how big the portions were, what meal you were eating, etc. The most important thing in your writing, however, is for you to describe what you ate since yesterday as accurately as possible. 
On the second day of writing

How did your writing go yesterday? In today's writing, I want you to describe what you did yesterday from the time you got up until the time you went to bed. I am not interested in your emotions or opinions, but rather I want you to write about specific objects or events in detail without discussing your thoughts or feelings. Feel free to be as detailed as possible. For example, you might start when your alarm went off and you got out of bed. You can include the things you ate, who you sat next to, where you went, which buildings or objects you passed as you walked from place to place. The most important thing in your writing, however, is for you to describe your days as accurately as possible.

On the third day of writing

Today, I would like you to describe what you have eaten since yesterday morning after you woke up, until the time that you came here for this study. Again, I want you to be as objective as possible and describe exactly what you have eaten up until coming to this experiment. For example, you might describe the texture, the color, an estimate of calories and fat, how big the portions were... The most important thing in your writing, however, is for you to describe what you ate since yesterday as accurately and as objectively as possible.

On the fourth day of writing

This is the last day of writing. In your writing today, I want you to describe what you have done today since you woke up. Again, I want you to be as objective as possible to describe exactly what you have done up until coming to this experiment... 


\section{Appendix B}

Please answer the questions below as accurately and honestly as possible.

What day did you come to Rubicon?

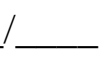

Today's date:

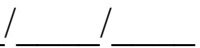

Your date of birth:

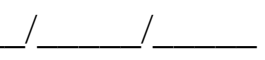

Mo. Day Yr.

Marital Status:

O Single / Never Married

O Married

Divorced/Separated

O Widowed

Race/Ethnicity:

O African - American

O White - American

O Asian - American

Hispanic

Other (specify):
Education:

Last grade completed:

Current Employment:

O $\square \square \square \square$ Unemployed

O Homemaker

O Disability

O Work Full Time (35 + hrs/week) Occupation:

Work Part Time (<35 hrs/week) Occupation:

O Student

At the present time, what is your religious preference?

Roman Catholic

O Protestant (Baptist, Episcopal, Lutheran, Methodist, Presbyterian, etc.)

O Jewish

Muslim

O Buddhist

Other (specify): 
Date:

Researcher:

Research ID:

\section{CES-D}

Below is a list of the ways you might have felt or behaved. Please check the appropriate box to tell how often you have felt this way during the past week.

\begin{tabular}{|l|l|l|l|}
\hline $\begin{array}{l}\text { Rarely or } \\
\text { none of the } \\
\text { time (less } \\
\text { than 1 day) }\end{array}$ & $\begin{array}{l}\text { Some or } \\
\text { a little of } \\
\text { the time } \\
(1-2 \\
\text { days })\end{array}$ & $\begin{array}{l}\text { Occasionally } \\
\text { or a } \\
\text { moderate } \\
\text { amount of } \\
\text { time (3-4 } \\
\text { days) }\end{array}$ & $\begin{array}{l}\text { Most or all } \\
\text { of the time } \\
\text { (5-7 days) }\end{array}$ \\
\hline
\end{tabular}

1. I was bothered by things that usually don't bother me.
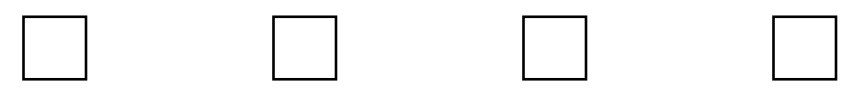

2. I did not feel like eating; my appetite was poor.
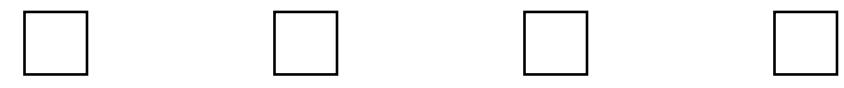

3. I felt that I could not shake off the blues even with help from my family
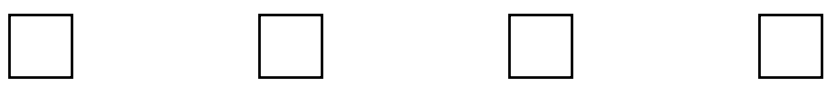
or friends.

4. I felt I was just as good as other people.
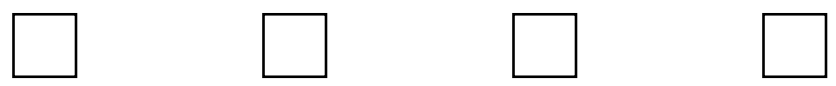

5. I had trouble keeping my mind on what

I was doing.
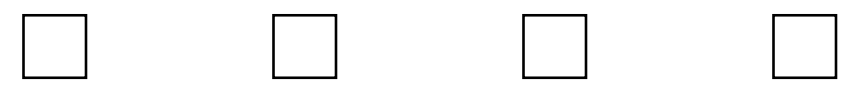

6. I felt depressed.
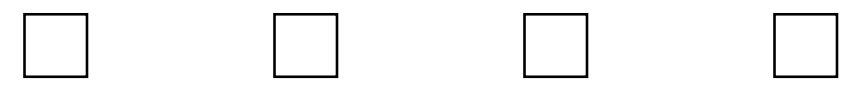

7. I felt that everything I did was an effort.
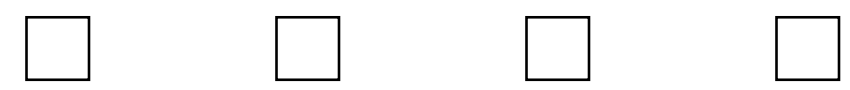

8. I felt hopeful about the future.

9. I thought my life had been a failure.
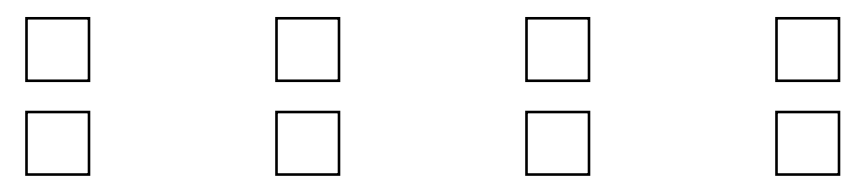


\begin{tabular}{|l|l|l|l|}
\hline $\begin{array}{l}\text { Rarely or } \\
\text { none of the } \\
\text { time (less } \\
\text { than 1 day) }\end{array}$ & $\begin{array}{l}\text { Some or } \\
\text { a little of } \\
\text { the time } \\
(1-2 \\
\text { days })\end{array}$ & $\begin{array}{l}\text { Occasionally } \\
\text { or a } \\
\text { moderate } \\
\text { amount of } \\
\text { time (3-4 } \\
\text { days) }\end{array}$ & $\begin{array}{l}\text { Most or all } \\
\text { of the time } \\
\text { (5-7 days) }\end{array}$ \\
\hline
\end{tabular}

10. I felt fearful.

11. My sleep was restless.

12. I was happy.
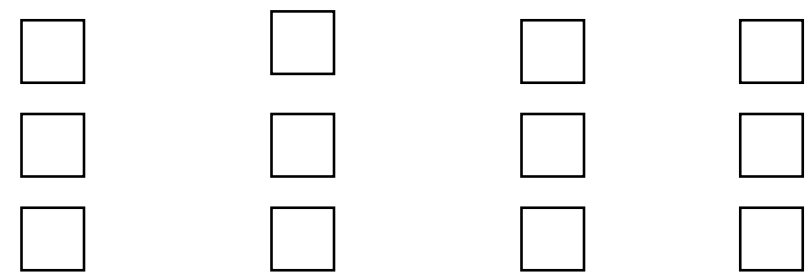

13. I talked less than usual.

14. I felt lonely.

15. People were unfriendly.
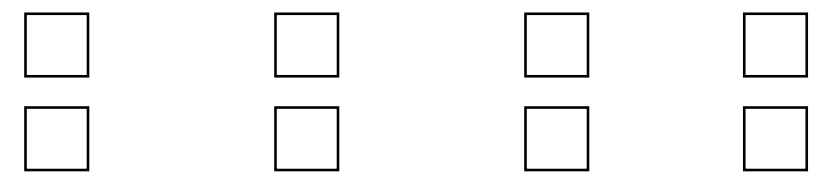

16. I enjoyed life.

17. I had crying spells.

18. I felt sad.

19. I felt that people disliked me.
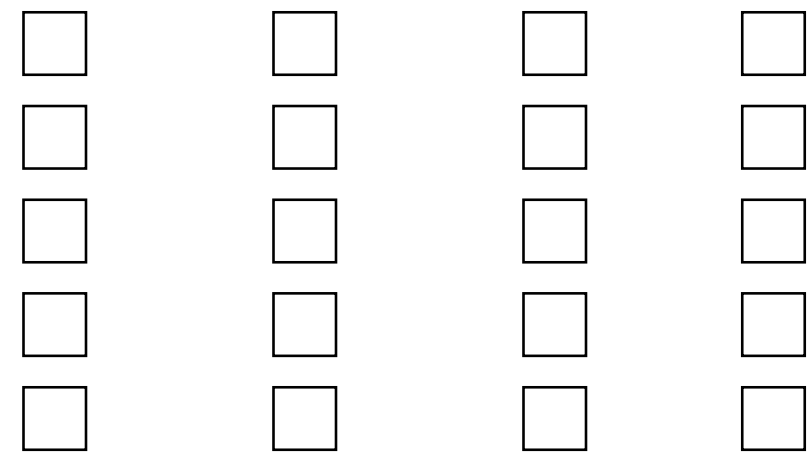

20. I could not get "going".
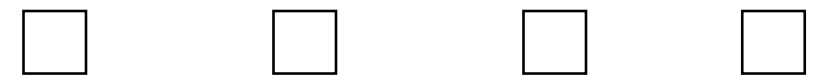


\section{PANAS}

\section{Directions}

This scale consists of a number of words that describe different feelings and emotions.

Read each item and then circle the appropriate answer next to that word. Indicate to what extent feel this way right now.

Use the following scale to record your answers.
(1) = Very slightly
(2) $=$ A little
(3) = Moderately
(4) = Quite a bit
(5) $=$ Extremely or not at all

\begin{tabular}{|l|c|c|c|c|c|}
\hline & $\begin{array}{c}\text { Very } \\
\text { slightly or } \\
\text { not at all }\end{array}$ & & Moderately & Quite a bit & \\
& & & & Extremely \\
\hline 1. Interested & 1 & 2 & 3 & 4 & 5 \\
\hline 2. Distressed & 1 & 2 & 3 & 4 & 5 \\
\hline 3. Excited & 1 & 2 & 3 & 4 & 5 \\
\hline 4. Upset & 1 & 2 & 3 & 4 & 5 \\
\hline 5. Strong & 1 & 2 & 3 & 4 & 5 \\
\hline 6. Guilty & 1 & 2 & 3 & 4 & 5 \\
\hline 7. Scared & 1 & 2 & 3 & 4 & 5 \\
\hline 8. Hostile & 1 & 2 & 3 & 4 & 5 \\
\hline 9. Enthusiastic & 1 & 2 & 3 & 4 & 5 \\
\hline 10. Proud & 1 & 2 & 3 & 4 & 5 \\
\hline 11. Irritable & 1 & 2 & 3 & 4 & 5 \\
\hline 12. Alert & 1 & 2 & 3 & 4 & 5 \\
\hline 13. Ashamed & 1 & 2 & 3 & 4 & 5 \\
\hline 14. Inspired & 1 & 2 & 3 & 4 & 5 \\
\hline 15. Nervous & 1 & 2 & 3 & 4 & 5 \\
\hline 16. Determined & 1 & 2 & 3 & 4 & 5 \\
\hline 17. Attentive & 1 & 2 & 3 & 4 & 5 \\
\hline
\end{tabular}




\begin{tabular}{|l|l|l|l|l|l|}
\hline 18. Jittery & 1 & 2 & 3 & 4 & 5 \\
\hline 19. Active & 1 & 2 & 3 & 4 & 5 \\
\hline 20. Afraid & 1 & 2 & 3 & 4 & 5 \\
\hline
\end{tabular}




\section{The PILL}

Several common symptoms or bodily sensations are listed below. Most people have experienced most of them at one time or another. We are currently interested in finding out how prevalent each symptom is among various groups of people. On the page below, write how frequently you experience each symptom. For all items, use the following scale:

\begin{tabular}{|l|l|l|l|l|}
\hline A & B & C & D & E \\
\hline $\begin{array}{l}\text { Have never or } \\
\text { almost never } \\
\text { experienced the } \\
\text { symptom }\end{array}$ & $\begin{array}{l}\text { Less than 3 or } \\
\text { times per year }\end{array}$ & $\begin{array}{l}\text { Every month or } \\
\text { so }\end{array}$ & $\begin{array}{l}\text { Every week } \\
\text { or so }\end{array}$ & $\begin{array}{l}\text { More than } \\
\text { once every } \\
\text { week }\end{array}$ \\
\hline
\end{tabular}

For example, if your eyes tend to water once every week or two, you would answer "D" next to question \#1.

1. Eyes water

2. Itchy eyes or skin

3. Ringing in ears

4. Temporary deafness or hard of hearing

5. Lump in throat

6. Choking sensations

7. Sneezing spells

8. Running nose

9. Congested nose

10. Bleeding nose

11. Asthma or wheezing

12. Coughing

13. Out of breath

14. Swollen ankles

15. Chest pains

16. Racing heart

17. Cold hands or feet even in hot weather

18. Leg cramps

19. Insomnia or difficulty sleeping

20. Toothaches
28. Swollen joints

29. Stiff or sore muscles

30. Back pains

31. Sensitive or tender skin

32. Face flushes

33. Tightness in chest

34. Skin breaks out in rash

35. Acne or pimples on face

36. Acne/pimples other than face

37. Boils

38. Sweat even in cold weather

39. Strong reactions to insect bites

40. Headaches

41. Feeling pressure in head

42. Hot flashes

43. Chills

44. Dizziness

45. Feel faint

46. Numbness or tingling in any part of body

47. Twitching of eyelid 
21. Upset stomach

22. Indigestion

23. Heartburn or gas

24. Abdominal pain

25. Diarrhea

26. Constipation

27. Hemorrhoids
48. Twitching other than eyelid

49. Hands tremble or shake

50. Stiff joints

51. Sore muscles

52. Sore throat

53. Sunburn

54. Nausea 
Brief Substance Craving Scale (BSCS)

Please answer the following questions with regard to Craving for Drug of Choice:

Drug of choice:

The INTENSITY of my craving, that is, how much I desired cocaine in the past 24 hours was:

O- 0 None at O-1 Slight O-2 Moderate $\quad$ O- 3 Considerable $\quad \mathrm{O}-4$

all Extreme

The FREQUENCY of my craving, that is, how often I thought of cocaine in the past 24 hours was:
O - 0 Never
O - 1 Almost Never
O - 2 Several
O - 3
Regularly
O - 4 Almost Constantly

The LENGTH of Time I spent in craving for cocaine during the past 24 hours was:
$\mathrm{O}$ - 0 None at
O - 1 Very
O - 2 Short
O - 3 Somewhat Long
O - 4 Very
all
Short
Long

\begin{tabular}{|c|c|c|c|}
\hline \multicolumn{4}{|c|}{ A 2nd craved drug during the past 24 hours was: $\quad$ (mark ONLY ONE of the following) } \\
\hline $\begin{array}{l}\text { O - Downers or Sedatives (Barbiturates } \\
\text { etc.) }\end{array}$ & $\begin{array}{l}\text { O - Benzos (Valium, Xanex, } \\
\text { etc.) }\end{array}$ & O - Nicotine & $\begin{array}{l}\mathrm{O}- \\
\text { Alcohol }\end{array}$ \\
\hline $\begin{array}{l}\text { O - Heroin or other Opiates (Morphine, } \\
\text { etc.) }\end{array}$ & O - Cocaine or Crack & $\begin{array}{l}\mathrm{O}- \\
\text { Marijuana }\end{array}$ & $\begin{array}{l}\text { O - } \\
\text { Others }\end{array}$ \\
\hline
\end{tabular}

The INTENSITY of my craving, that is, how much I desired this second drug in the past 24 hours was:
$\mathrm{O}$ - 0 None at
O - 1 Slight
O - 2 Moderate
O - 3 Considerable
O - 4

all

The FREQUENCY of my craving, that is, how often I thought of this second drug in the past 24 hours was:

O- 0 Never O-1 Almost Never O-2 Several Times O-3 Regularly O - 4 Almost

Constantly

The LENGTH of Time I spent in craving for this second drug during the past 24 hours was:

O- 0 None at all O-1 Very Short O-2 Short O-3 Somewhat Long O - 4 Very

Long

\begin{tabular}{|c|c|c|c|}
\hline \multicolumn{4}{|c|}{ A 3rd craved drug during the past 24 hours was: $\quad$ (mark ONLY ONE of the following) } \\
\hline $\begin{array}{l}\text { O - Downers or Sedatives (Barbiturates } \\
\text { etc.) }\end{array}$ & $\begin{array}{l}\text { O - Benzos (Valium, Xanex, } \\
\text { etc.) }\end{array}$ & O - Nicotine & $\begin{array}{l}\mathrm{O}- \\
\text { Alcohol }\end{array}$ \\
\hline $\begin{array}{l}\text { O - Heroin or other Opiates (Morphine, } \\
\text { etc.) }\end{array}$ & O - Cocaine or Crack & $\begin{array}{l}\mathrm{O} \text { - } \\
\text { Marijuana }\end{array}$ & $\begin{array}{l}\mathrm{O}- \\
\text { Others }\end{array}$ \\
\hline
\end{tabular}

The INTENSITY of my craving, that is, how much I desired this third drug in the past 24 hours was:
O - 0 None at
O - 1 Slight
O - 2 Moderate
O - 3 Considerable
$\mathrm{O}-4$
all

The FREQUENCY of my craving, that is, how often I thought of this third drug in the past 24 hours was:
O - 0 Never
O - 1 Almost Never
O - 2 Several Times
O - 3 Regularly
O - 4 Almost
Constantly

The LENGTH of Time I spent in craving for this third drug during the past 24 hours was:

O- 0 None at all O-1 Very Short O-2 Short O-3 Somewhat Long O - 4 Very Long 
Please indicate the extent to which the following descriptions describe the essay you just wrote. Circle the number that best describes your writing for the following statements:

1. The essay was meaningful.

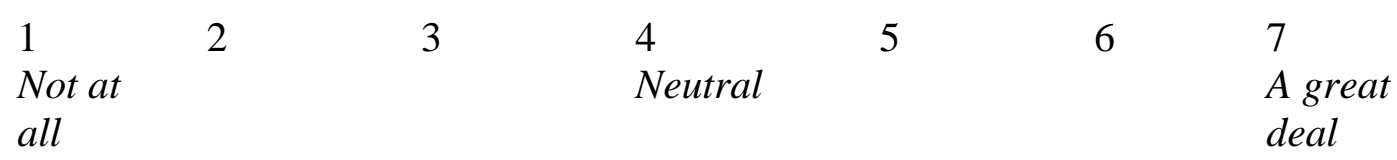

2. The essay was personal.

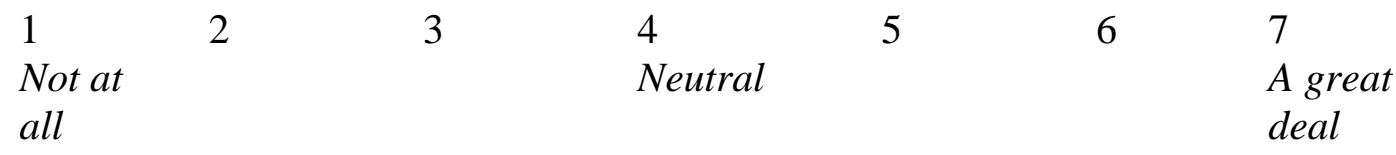

3. The essay was revealing of my emotions.

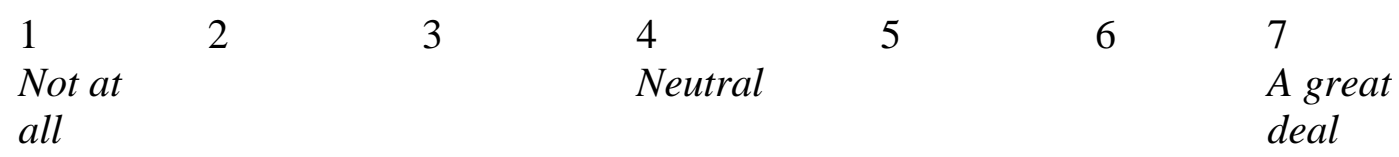

4. I wanted to talk to others about the event(s) that I wrote about.

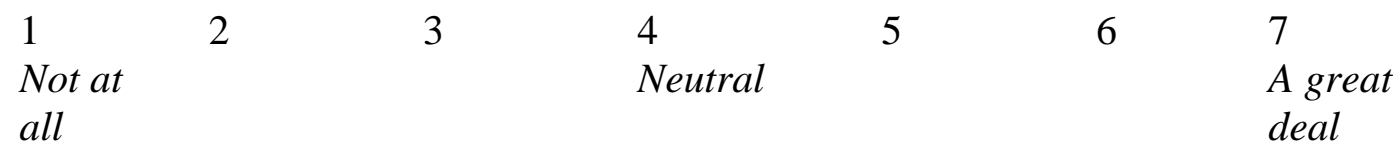

5. I have actually talk to others about the event(s) that I wrote about.

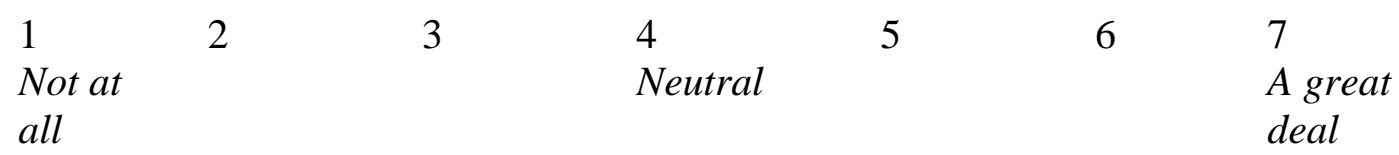

6. I have actively held back from talking to others about the event(s) that I wrote about.

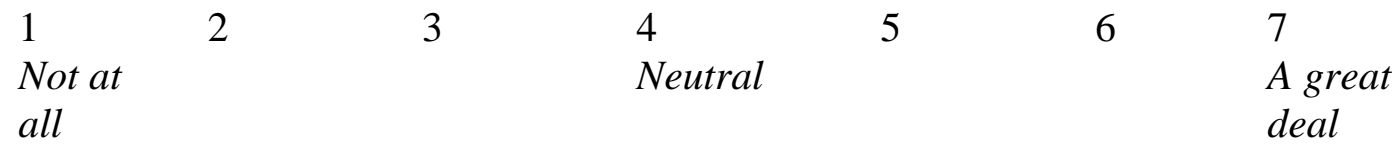




\section{Follow Up Questionnaire}

1. Did you hear any clients at Rubicon discussing the writing instructions for this study? (please put a check next to your response)

Yes _ No

2. What do you think the other writing instructions were about? (please put your answer below)

3. Did you tell any other clients at Rubicon about the writing instructions you were given for this study?

Yes _ No

4. Did you find the writing helpful? Why? Why not? (please put your answer below)

5. Did you talk about the subject matter from your essays with anyone during your treatment at Rubicon?

Yes _ No

6. a) If you answer 'Yes' to \#5 -- Did you find it helpful to talk about?

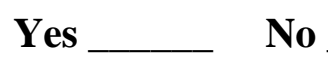

7. Did you continue writing, using the writing instructions given to you during this study, after the writing sessions had ended?

Yes _ No

Thank you! 


\section{Vita}

Sarah Meshberg-Cohen was born in Connecticut, and is an American Citizen. She graduated from Fairfield High School in Fairfield, CT in 1998. She received her Bachelor of Arts in Psychology from Skidmore College in Saratoga Springs, NY in 2002. In 2004, she enrolled in the Clinical Psychology Doctoral Degree program at Virginia Commonwealth University in Richmond, VA, where she received a Master of Sciences in May 2006. After completing a clinical internship at Yale University in New Haven, CT, Sarah will graduate in May 2010 with her doctorate in Clinical Psychology. 